Pure and Applied Mathematics Quarterly

Volume 5, Number 3

(Special Issue: In honor of

Friedrich Hirzebruch, Part 2 of 2$)$

977-1028, 2009

\title{
The Secondary Spherical Functions and Automorphic Green Currents for Certain Symmetric Pairs
}

\author{
Takayuki Oda and Masao Tsuzuki \\ Dedicated to Professor Friedrich Hirzebruch on his 80th birthday
}

\begin{abstract}
Given a modular embedding $j: \Delta \backslash H / H \cap K \rightarrow \Gamma \backslash G / K$ associated with an equivariant embedding $(H, H / H \cap K) \rightarrow(G, G / K)$ of symmetric domains with actions of a semisimple Lie group $G$ and a reductive subgroup $H$, both defined over $\mathbb{Q}$ compatibly, together with some other conditions. Starting from a certain harmonic left $H$-invariant "spherical" current on $G / K$ with sigularity along $H K / K$, we can define a Poincaré series. Apply $\partial \bar{\partial}$ operator to the analytic continutation of this with respect to the parameter of eigenvalues of the "Laplacian". Then as an analogue of the Kronecker limit formula, we can construct a Green current for the cycle defined by $j$. This is a continuation of the previous paper [26], and here we treat the case of higher-codimensional cycles with compact $\Gamma \backslash G / K$.
\end{abstract}

Keywords: Green current, modular embedding, modular cycle, spherical functions.

\section{INTRODUCTION}

Arithmetic quotients of hermitian symmetric domains are important objects to investigate. For example, the moduli spaces of abelian varieties with certain endomorphisms and polarization types, and the moduli spaces of K3 surfaces, are realized as such. To understand the cohomology groups and the cycle geometry 
of these quotients is a very interesting arithmetic problem. There is a history of investigating this theme around the time of establishment of the Matsushima isomorphism. The construction method of cycles by means of equivariant embeddings of locally symmetric spaces is called 'generalized modular symbols '. ( $c f$. $[18])$.

If both the embedded and the ambient spaces are of hermitian type, there is an extensive study by Satake [30] for possible embeddings. Sometimes they have been called modular embeddings. The Hirzebruch-Zagier cycles are typical examples ([12], [23]). Let

$$
j: \Delta \backslash H / H \cap K \rightarrow \Gamma \backslash G / K
$$

be a modular embedding with $G$ a semisimple Lie group, $K$ a maximal compact subgroup of $G, H$ a symmetric subgroup such that $H \cap K$ is maximally compact in $H$, and $\Gamma, \Delta$ are compatible arithmetic subgroups of $G, H$ respectively. Let $n$ and $m$ be dimensions of the symmetric spaces $H / H \cap K$ and $G / K$ respectively. Then, for each degree $q, j$ yields the restriction map of cohomology

$$
j_{\odot}^{q}: \mathrm{H}_{\odot}^{q}(\Gamma \backslash G / K, \mathbb{C}) \rightarrow \mathrm{H}_{\odot}^{q}(\Delta \backslash H / H \cap K, \mathbb{C}),
$$

where $\varnothing \in\{$ empty, c, ! $\}$ is a support condition of cohomology theories. Then we have the Poincaré dual map

$$
j_{\boldsymbol{\uparrow}}^{q}: \mathrm{H}_{\boldsymbol{\wedge}}^{m-q}(\Delta \backslash H / H \cap K, \mathbb{C}) \rightarrow \mathrm{H}_{\boldsymbol{\uparrow}}^{n-q}(\Gamma \backslash G / K, \mathbb{C})
$$

with the support condition dual to $\odot$. We propose here a

Problem: Construct the Poincaré dual map $j_{\text {a }}^{q}$ explicitly.

This problem seems to be quite difficult to answer generally. But at least for some special cases, we have a tractable method: to use Poincaré series and derived Green currents.

In a previous paper [26], we discuss the case when the complex codimension of $\Delta \backslash H / H \cap K$ in $\Gamma \backslash G / K$ is one. We can extend the similar construction for the higher codimensional case associated with the symmetric pair $\mathrm{U}(p, q), \mathrm{U}(p-$ $1, q) \times \mathrm{U}(1)$ in this article. We note that its dual symmetric pair $\mathrm{U}(p+q-$ $1,1), \mathrm{U}(p-1,1) \times \mathrm{U}(q)$, which yields a class of higher codimensional cycles in a discrete quotient of a complex hyperball, is already treated in [32] by a similar method. This paper is a continuation of our previous works [26] and [32], which we follow technically and logically. 
Let us explain the organization of this paper briefly. The aim of this paper is twofold: one is a local investigation of the secondary spherical function $(\S \S 4-5)$, and the other is a global investigation of the associated Poincaré series ( $\S \S 6-8)$. Our spherical functions are left $H$-invariant smooth functions on $G$ which have special right $K$-types and are eigenfunctions under the Casimir operator. The secondary spherical functions have similar properties, but they are distributions on $G$ with singularities along $H K$. These kinds of functions play a crucial role in our construction of Poincaré series and Green currents.

Here is a more detailed explanation of each section. The second section is preliminary, where we fix basic notations and assemble relevant facts about our symmetric pair $(G, H)=(\mathrm{U}(p, q), \mathrm{U}(p-1, q) \times \mathrm{U}(1))$, and also fix a normalization of Haar measures. In $\S 3$, we study a certain invariant tensor associated with the submanifold $H / H \cap K$ by means of representation theory of compact groups. The $\S 4$ is an analytical preliminary, where we give a concrete expression of the Hodge Laplacian and the operator $\partial \bar{\partial}$ on the symmetric space $G / K$ in terms of the 'polar coordinates' on $H \backslash G$. The secondary spherical function is studied in $\S 5$. In Theorem 18, we define the secondary spherical function $\varphi_{s}^{(d)}$ for each $0 \leqslant$ $d \leqslant q$ as a family of $H$-invariant $(d, d)$-forms on $G / K$ with singularities along the submanifold $H / H \cap K$ which holomorphically depends on a complex parameter $s$ and satisfies the five characterizing conditions (i)-(v). We explicitly construct such a family by using the Gaussian hypergeometric series. The characterizing conditions in Theorem 18 are effectively used to prove the equation in Theorem 26, which is important to show the Green's equation (7.1) in $\S 7$ Theorem 35.

The remaining sections are occupied by the investigation of global currents. We study a modular cycle $C_{H}^{\Gamma}: \Gamma \cap H \backslash H / H \cap K \rightarrow \Gamma \backslash G / K$ defined by a uniform lattice $\Gamma$ of $G$ such that $\Gamma \cap H \backslash H$ is also compact. In order to obtain the Poincaré dual of $C_{H}^{\Gamma}$, in $\S 6$, we define a $(q, q)$-current $\Psi_{H}^{\Gamma}(s)=\Phi_{s, 0}^{(q)}$ and the related $(d, d)$ currents $\Phi_{s, r}^{(d)}$ on $\Gamma \backslash G / K$ as the Poincaré series using the secondary spherical function $\varphi_{s}^{(d)}$ as the seeds of the 'geometric current ' constructed in the last part of the paper. After establishment of its $L^{1}$-convergence in the range $\operatorname{Re}(s)>p+q-1$, in Proposition 31, we show the generalized Poisson equation for $\Phi_{s, r}^{(d)}$.

Although the form $\Psi_{H}^{\Gamma}(s)$ is not square-integrable itself, we can establish the square-integrability for the auxiliary currents $\Phi_{s, r}^{(d)}$ with sufficiently large $r$ by a similar way to [26]. We obtain the spectral decomposition of the square-integrable 
form $\Phi_{s, r}^{(d)}$ as a Fourier series of the eigenfunction of the Hodge Laplacian, which eventually yields the meromorphic continuation of $\Psi_{H}^{\Gamma}(s)$ from the original convergence region $\operatorname{Re}(s)>p+q-1$ to the whole complex plane.

In $\S 7$, we establish Green's equation (7.1) in Theorem 35, which, together with the generalized Poisson equation, is used in Theorem 36 to show the current $\Psi_{H}^{\Gamma}=$ $\frac{p+q-1}{2} \operatorname{Res}_{s=p+q-1} \Psi_{H}^{\Gamma}(s)$ is harmonic and is cohomologus to the fundamental class of $C_{H}^{\Gamma}$. We also show that the constant term of $\Phi_{s, 0}^{(q-1)}$ at $s=p+q-1$ yields a Green current of $C_{H}^{\Gamma}$ in the sense of Gillet-Soulé [5], though the conditions at singularities are different.

In $\S 8$, we study some representation theoretical aspects of our global construction $\Psi_{H}^{\Gamma}$. We collect miscellaneous remarks and perspectives related to the theme of this paper in $\S 9$.

Finally, we should say a few words about existing works related to the theme of this article.

When the complex codimension of $H / H \cap K$ in $G / K$ is one, the modular construction of a Green current of $C_{H}^{\Gamma}$ is obtained in [26] in the same way as this paper. If $G / K$ and $H / H \cap K$ are type IV symmetric domains and if $\Gamma$ is a discriminant group of some rational quadratic form, Bruinier [2] constructed a Green function for a 'Heegner divisor' (which is a member of the divisor class group of $\Gamma \backslash G / K$ expressed as a linear combination of $C_{H_{i}}^{\Gamma}$ for various $H_{i}$ 's defined over $\mathbb{Q}$ ) by a 'regularized theta lifting'. It turns out the Green function in [2] is built from the one in [26] according to the formation of the relevant Heegner divisor.

Though the relation is somewhat indirect from technical point of view, there are some results strongly related from the geometric point of view, which are worked in the context of Weil representation and the theta correspondence ([23], $[24],[14],[15],[4],[16],[17])$. To explain the connection in detail takes some space; therefore, we leave that to the readers.

The paper [13] is also related to this paper. There we also considered modular symbols derived from the injection $H \hookrightarrow G$ for a symmetric pair $(G, H)$. But in this case the image of the locally symmetric space associated with the subgroup $H$ is totally real, in contrast with the fact that here in this paper we consider the holomorphic embedding. Actually we have some evidence to believe that the 
modular symbols considered in [13] have the extremal Hodge components (i.e., $(m, 0)$-type components), but our cycles are algebraic hence have only $(p, p)$-type Hodge components. In this sense, the two results in this paper and [13] might be the two edges of some more general phenomena.

Based on a work of Oshima-Matsuki, Tong-Wang [33] provides a fairly general and simple method to construct an automorphic realization of a discrete series of a symmetric space, which yields a modular construction of the Poincaré dual form associated with a cohomology class defined by the symmetric subgroup in a cohomology group with a local coefficient system. For analytical reasons, they need to assume that the coefficient system should be sufficiently regular. The assumption is related to the $L^{1}$-condition of the discrete series, which is indispensable to guarantee the convergence of the Poincaré series they use. This is a serious technical limitation to obtaining the Poincaré dual forms in the cohomology with constant coefficient. To be more concrete, let us pick the representation $A_{\mathfrak{q}_{1 p}}$ defined in $\S 8$. It is easy to see that $A_{\mathfrak{q}_{1 p}}$ is not integrable; so one can not expect the convergence of the Poincaré series ' $\sum_{\gamma \in \Gamma \cap H \backslash \Gamma} \psi_{H}(\gamma g)$ ' used in [33]. Though the secondary spherical function $\psi_{s}$ has a singularity, it will be shown in this papaer that $\psi_{s}$ is good enough to assure the convergence of the Poincaré series $\Psi_{H}^{\Gamma}(s ; g)=\sum_{\gamma \in \Gamma \cap H \backslash \Gamma} \psi_{s}(\gamma g)$ for large $\operatorname{Re}(s)$. We can recover the object ' $\sum_{\gamma \in \Gamma \cap H \backslash \Gamma} \psi_{H}(\gamma g)$ ' properly by taking the residue at $s=p+q-1$ after the meromorphic continuation of the series $\Psi_{H}^{\Gamma}(s)$. This regularization procedure reminds us of the 'Hecke's trick' which is used to obtain an Eisenstein series with low weight in the classical theory of elliptic modular forms ([37]). In this analogy, the construction of the automorphic Green current can be regarded as a kind of the second limit formula of Kronecker ([37]).

Acknowledgement: The authors thank Professor Eric Stade for linguistic help.

\section{Notations:}

The number 0 is included in the set of natural numbers: $\mathbb{N}=\{0,1,2, \ldots\}$. We understand all the marix in this paper have complex coefficients. For any matrix $B=\left(b_{i j}\right), B^{*}=\left(\bar{b}_{j i}\right)$ denotes its conjugate-transpose matrix. For a poistive integer $p, 1_{p}$ denotes the identity matrix of size $p$. For $r$ matrices $A_{j}(1 \leqslant j \leqslant r)$, $\operatorname{diag}\left(A_{1}, \ldots, A_{r}\right)$ deontes the 'block diagonal matrix 'corresponding to the linear endomorphism $A_{1} \oplus \cdots \oplus A_{r}$. 
We follow the usual convention that the Lie algebra of a real Lie group $G$ is denoted by the corresponding German letter $\mathfrak{g}$.

\section{Preliminaries}

2.1. Unitary group and its symmetric space. Let $G$ be the unitary group of the Hermitian form $I_{p, q}=\operatorname{diag}\left(1_{p},-1_{q}\right)$ with signature $(p+, q-)$, i.e., $G=\{g \in$ $\left.\mathrm{GL}_{p+q}(\mathbb{C}) \mid g^{*} I_{p, q} g=I_{p, q}\right\}$. We assume $p \geqslant q \geqslant 2$ from now on.

The inner automorphism $\theta: g \mapsto I_{p, q} g I_{p, q}$ is a Cartan involution of $G$ and its fixed point set

$$
K=\left\{\operatorname{diag}\left(k_{1}, k_{2}\right) \mid k_{1} \in \mathrm{U}(p), k_{2} \in \mathrm{U}(q)\right\}
$$

yields a maximal compact subgroup of $G$. The (-1)-eigenspace of $d \theta: \mathfrak{g} \rightarrow \mathfrak{g}$ denoted by $\mathfrak{p}$ is identified with the tangent space of the $G$-homogeneous manifold $G / K$ at its origin $o=K$. The adjoint action $J=\operatorname{Ad}\left(z_{o}\right) \mid \mathfrak{p}$ by the element $z_{o}=\operatorname{diag}\left(\sqrt{-1} 1_{p}, 1_{q}\right)$ in the center of $K$ yields a $K$-invariant complex structure on $\mathfrak{p} \cong T_{o}(G / K)$, which propagates a $G$-invariant complex structure on $G / K$. The complexification $\mathfrak{p}_{\mathbb{C}}$ is decomposed to its holomorphic and anti-holomorphic subspaces: $\mathfrak{p}_{\mathbb{C}}=\mathfrak{p}_{+} \oplus \mathfrak{p}_{-}$with $\mathfrak{p}_{ \pm}=\left\{X \in \mathfrak{p}_{\mathbb{C}} \mid J(X)= \pm \sqrt{-1} X\right\}$. If we identify $\mathfrak{g}_{\mathbb{C}}=\mathfrak{g l}_{p+q}(\mathbb{C})$ naturally, we have

$$
\begin{aligned}
& \mathfrak{p}_{+}=\left\{p_{+}\left(x^{\prime}\right)=\left[\begin{array}{cc}
0 & x^{\prime} \\
0 & 0
\end{array}\right] \in \mathfrak{g l}_{p+q}(\mathbb{C}) \mid x^{\prime} \in \mathrm{M}_{p, q}(\mathbb{C})\right\}, \\
& \mathfrak{p}_{-}=\left\{p_{-}\left(x^{\prime \prime}\right)=\left[\begin{array}{cc}
0 & 0 \\
{ }^{\prime \prime} x^{\prime \prime} & 0
\end{array}\right] \in \mathfrak{g l}_{p+q}(\mathbb{C}) \mid x^{\prime \prime} \in \mathrm{M}_{p, q}(\mathbb{C})\right\} .
\end{aligned}
$$

Let $X \mapsto \bar{X}$ be the complex conjugate in $\mathfrak{g l}_{p+q}(\mathbb{C})$ with respect to its real form $\mathfrak{g}$. Then $\bar{X}=-I_{p, q} X^{*} I_{p, q}\left(\forall X \in \mathfrak{g}_{\mathbb{C}}\right)$ and $\overline{p_{ \pm}(x)}=p_{\mp}\left({ }^{t} x^{*}\right)\left(\forall x \in \mathrm{M}_{p, q}(\mathbb{C})\right)$. The nondegenerate $\mathbb{R}$-bilinear form $B_{\mathfrak{g}}(X, Y)=2^{-1} \operatorname{tr}(X Y)$ on $\mathfrak{g}$ entails a positive definite $K$-invariant inner product $B_{\mathfrak{p}}$ on $\mathfrak{p}$, which propagates a $G$-invariant metric on $G / K$. The mertic on $G / K$ is Kählerian and the associated 2-form is given by

$$
\omega_{\mathfrak{p}}(X, Y)=B_{\mathfrak{p}}(X, J Y), \quad X, Y \in \mathfrak{p}
$$

on $\mathfrak{p} \cong T_{o}(G / K)$. Let $B_{\mathfrak{p}_{\mathbb{C}}^{*}}$ be the complex bilinear extension of the inner product $B_{\mathfrak{p}^{*}}$ on $\mathfrak{p}^{*}$ dual to $B_{\mathfrak{p}}$. Then $\mathfrak{p}_{\mathbb{C}}^{*}$ is equipped with the hermitian inner product $\left(\xi \mid \xi^{\prime}\right)=B_{\mathfrak{p}_{\mathbb{C}}^{*}}\left(\xi, \bar{\xi}^{\prime}\right)$, which is extended to the exterior algebra $\bigwedge \mathfrak{p}_{\mathbb{C}}^{*}$ canonically. Note the natural decomposition of $\wedge \mathfrak{p}_{\mathbb{C}}^{*}$ to its bidegree $(a, b)$ part

$$
\bigwedge^{a, b} \mathfrak{p}_{\mathbb{C}}^{*}=\bigwedge^{a} \mathfrak{p}_{+}^{*} \otimes \bigwedge^{b} \mathfrak{p}_{-}^{*}
$$

is orthogonal. 
The Hodge star operator $*$ is defined to be the $\mathbb{C}$-linear endomorphism of $\bigwedge \mathfrak{p}_{\mathbb{C}}^{*}$ such that $* \bar{\alpha}=\overline{* \alpha}$ and such that $(\alpha \mid \beta) \operatorname{vol}_{\mathfrak{p}}=\alpha \wedge * \bar{\beta}$. Here $\operatorname{vol}_{\mathfrak{p}}=\frac{1}{(p q) !} \omega_{\mathfrak{p}}^{p q}$ is the Kähler volume form. For $\alpha \in \wedge \mathfrak{p}_{\mathbb{C}}^{*}$, let us define $e(\alpha): \wedge \mathfrak{p}_{\mathbb{C}}^{*} \rightarrow \wedge \mathfrak{p}_{\mathbb{C}}^{*}$ by $e(\alpha) \beta=\alpha \wedge \beta$. The operator $L=e\left(\omega_{\mathfrak{p}}\right)$ is commonly called the Lefschetz operator. The adjoint of $e(\alpha)$ with respect to the hermitian inner product of $\bigwedge \mathfrak{p}_{\mathbb{C}}^{*}$ is denoted by $e^{*}(\alpha)$. In particular, the operator $e^{*}\left(\omega_{\mathfrak{p}}\right)$, the adjoint of the Lefschetz operator, is denoted by $\Lambda$ (cf. [1, Chap.II, §4]

2.2. A symmetric subgroup. Let us consider the involution $\sigma$ of $G$ defined by

$$
\sigma(g)=\operatorname{diag}\left(1_{p-1},-1,1_{q}\right) g \operatorname{diag}\left(1_{p-1},-1,1_{q}\right) .
$$

Let $H=G^{\sigma}$ be the $\sigma$-fixed point subgroup of $G$. Since $\theta$ commutes with $\sigma$, the restriction $\theta \mid H$ provides $H$ with a Cartan involution. The $\theta$-fixed points

$$
H^{\theta}=H \cap K=\left\{\operatorname{diag}\left(h_{1}, u, h_{2}\right) \mid h_{1} \in \mathrm{U}(p-1), u \in \mathrm{U}(1), h_{2} \in \mathrm{U}(q)\right\}
$$

is a maximal compact subgroup of $H$. The Cartan decomposition of the Lie algebra $\mathfrak{h}$ of $H$ is $\mathfrak{h}=(\mathfrak{k} \cap \mathfrak{h}) \oplus(\mathfrak{p} \cap \mathfrak{h})$. Since the element $z_{o}$ defining the complex structure $J$ of $\mathfrak{p}$ belongs to the center of $H \cap K, J$ yields an $H \cap K$-invariant complex structure of the real vector space $\mathfrak{h} \cap \mathfrak{p} \cong T_{o}(H / H \cap K)$, which propagates an $H$-invariant complex structure of $H$-homogeneous manifold $H / H \cap K$. We put $H / H \cap K$ the $H$-invariant metric coming from the restriction of $B_{\mathfrak{p}}$ to $\mathfrak{h} \cap \mathfrak{p}$. The metric is Kählerian and the associated 2-form on $\mathfrak{p} \cap \mathfrak{h} \cong T_{o}(H / H \cap K)$ is $\omega_{\mathfrak{p} \cap \mathfrak{h}}=\omega_{\mathfrak{p}} \mid(\mathfrak{p} \cap \mathfrak{h}) \times(\mathfrak{p} \cap \mathfrak{h})$.

As a consequence of the constructions so far, the inclusion $H / H \cap K \hookrightarrow G / K$ is a holomorphic map between Kähler manifolds and $\operatorname{codim}_{\mathbb{C}}(G / K ; H / H \cap K)=q$.

In the following subsection, we recall the standard set up to investigate the affine symmetric pair $(G, H)$ (cf. Rossmann [29], Oshima-Sekiguchi [28] and Schlichtkrull [31]).

2.3. Root vectors. For $1 \leqslant i, j \leqslant p+q$, let $E_{i, j}=\left(\delta_{i \alpha} \delta_{j \beta}\right)_{1 \leqslant \alpha, \beta \leqslant p+q}$ denotes the matrix unit in $\mathrm{M}_{p+q}(\mathbb{C})$. The matrices $E_{i, j}$ comprise a $\mathbb{C}$-basis of the complexified Lie algebra $\mathfrak{g}_{\mathbb{C}}=\mathfrak{g l}_{p+q}(\mathbb{C})$.

Let $\mathfrak{q}$ be the $(-1)$-eigenspace of $d \sigma: \mathfrak{g} \rightarrow \mathfrak{g}$. Since $\theta$ and $\sigma$ are mutually commutative involutions, $\mathfrak{g}$ is decomposed to their joint eigenspaces: $\mathfrak{g}=(\mathfrak{k} \cap$ $\mathfrak{h}) \oplus(\mathfrak{p} \cap \mathfrak{q}) \oplus(\mathfrak{p} \cap \mathfrak{h}) \oplus(\mathfrak{k} \cap \mathfrak{q})$. The pair $(\mathfrak{g}, \mathfrak{h})$ is a symmetric pair of split rank 
one, and $\mathfrak{a}=\mathbb{R} Y_{0}$ with $Y_{0}=E_{p, p+1}+E_{p+1, p}$ is a maximal abelian subspace of $\mathfrak{p} \cap \mathfrak{q}$. The set of $\mathfrak{a}$-roots in $\mathfrak{g}$ is $\Sigma(\mathfrak{a})=\{ \pm \lambda, \pm 2 \lambda\}$. Here $\lambda \in \mathfrak{a}^{*}$ is the unique simple root such that $\lambda\left(Y_{0}\right)=1$. The multiplicity ([28]) of each root is computed as $\left(\begin{array}{l}m^{+}(\lambda) m^{+}(2 \lambda) \\ m^{-}(\lambda) m^{-}(2 \lambda)\end{array}\right)=\left(\begin{array}{ll}2(q-1) & 1 \\ 2(p-1) & 0\end{array}\right)$.

Set $M=Z_{H \cap K}(\mathfrak{a})$. Then

$$
M=\left\{\operatorname{diag}\left(x_{1}, u, u, x_{2}\right) \mid x_{1} \in \mathrm{U}(p-1), u \in \mathrm{U}(1), x_{2} \in \mathrm{U}(q-1)\right\}
$$

coincides with $Z_{K}(\mathfrak{a})$.

For $1 \leqslant i \leqslant p-1$ and $1 \leqslant j \leqslant q-1$, set

$$
\begin{array}{rlrl}
X_{0}^{\mathfrak{q}} & =E_{p, p+1}, & \bar{X}_{0}^{\mathfrak{q}} & =E_{p+1, p}, \quad Z_{0}^{\mathfrak{h}}=\sqrt{-1}\left(E_{p, p}-E_{p+1, p+1}\right), \\
X_{j}^{\mathfrak{q}} & =E_{p, p+j+1}, & \bar{X}_{j}^{\mathfrak{q}} & =E_{p+j+1, p}, \\
Z_{j}^{\mathfrak{h}} & =-E_{p+1, p+j+1}, \bar{Z}_{j}^{\mathfrak{h}}=E_{p+j+1, p+1}, \\
X_{i}^{\mathfrak{h}} & =E_{i, p+1}, & \bar{X}_{i}^{\mathfrak{h}} & =E_{p+1, i}, \\
Z_{i}^{\mathfrak{q}} & =E_{i, p}, & \bar{Z}_{i}^{\mathfrak{q}} & =-E_{p, i}, \\
X_{i j}^{\mathfrak{h}} & =E_{i, p+j+1}, & \bar{X}_{i j}^{\mathfrak{h}} & =E_{p+j+1, i} .
\end{array}
$$

This notation is consistent with the complex conjugation, and

$$
\begin{aligned}
& \mathfrak{p}_{+} \cap \mathfrak{q}_{\mathbb{C}}=\left\langle X_{j}^{\mathfrak{q}}(0 \leqslant j \leqslant q-1)\right\rangle_{\mathbb{C}}, \\
& \mathfrak{p}_{-} \cap \mathfrak{q}_{\mathbb{C}}=\left\langle\bar{X}_{j}^{\mathfrak{q}}(0 \leqslant j \leqslant q-1)\right\rangle_{\mathbb{C}}, \\
& \mathfrak{p}_{+} \cap \mathfrak{h}_{\mathbb{C}}=\left\langle X_{i}^{\mathfrak{h}}(1 \leqslant i \leqslant p-1)\right\rangle_{\mathbb{C}} \oplus\left\langle X_{i j}^{\mathfrak{h}}(1 \leqslant i \leqslant p-1,1 \leqslant j \leqslant q-1)\right\rangle_{\mathbb{C}}, \\
& \mathfrak{p}_{-} \cap \mathfrak{h}_{\mathbb{C}}=\left\langle\bar{X}_{i}^{\mathfrak{h}}(1 \leqslant i \leqslant p-1)\right\rangle_{\mathbb{C}} \oplus\left\langle\bar{X}_{i j}^{\mathfrak{h}}(1 \leqslant i \leqslant p-1,1 \leqslant j \leqslant q-1)\right\rangle_{\mathbb{C}}, \\
& (\mathfrak{k} \cap \mathfrak{h})_{\mathbb{C}}=\left\langle Z_{0}^{\mathfrak{h}}, Z_{j}^{\mathfrak{h}}, \bar{Z}_{j}^{\mathfrak{h}}(1 \leqslant j \leqslant q-1)\right\rangle_{\mathbb{C}} \oplus \mathfrak{m}_{\mathbb{C}}, \\
& (\mathfrak{k} \cap \mathfrak{q})_{\mathbb{C}}=\left\langle Z_{i}^{\mathfrak{q}}, \bar{Z}_{i}^{\mathfrak{q}}(1 \leqslant i \leqslant p-1)\right\rangle_{\mathbb{C}} .
\end{aligned}
$$

Here is a list of useful bracket relations, which is checked by a direct computation: For $1 \leqslant i \leqslant p-1$ and $1 \leqslant j \leqslant q-1$,

$$
\begin{aligned}
& {\left[Z_{0}^{\mathfrak{h}}, X_{0}^{\mathfrak{q}}\right]=2 \sqrt{-1} X_{0}^{\mathfrak{q}},\left[Z_{0}^{\mathfrak{h}}, \bar{X}_{0}^{\mathfrak{q}}\right]=-2 \sqrt{-1} \bar{X}_{0}^{\mathfrak{q}},\left[X_{0}^{\mathfrak{q}}, \bar{X}_{0}^{\mathfrak{q}}\right]=-\sqrt{-1} Z_{0}^{\mathfrak{h}},} \\
& {\left[Z_{0}^{\mathfrak{h}}, Z_{j}^{\mathfrak{h}}\right]=-\sqrt{-1} Z_{j}^{\mathfrak{h}},\left[\bar{X}_{0}^{\mathfrak{q}}, Z_{j}^{\mathfrak{h}}\right]=0, \quad\left[X_{0}^{\mathfrak{q}}, Z_{j}^{\mathfrak{h}}\right]=-X_{j}^{\mathfrak{q}},} \\
& {\left[Z_{0}^{\mathfrak{h}}, X_{j}^{\mathfrak{q}}\right]=\sqrt{-1} X_{j}^{\mathfrak{q}}, \quad\left[\bar{X}_{0}^{\mathfrak{q}}, X_{j}^{\mathfrak{q}}\right]=-Z_{j}^{\mathfrak{h}}, \quad\left[X_{0}^{\mathfrak{q}}, X_{j}^{\mathfrak{q}}\right]=0,} \\
& {\left[Z_{0}^{\mathfrak{h}}, Z_{i}^{\mathfrak{q}}\right]=-\sqrt{-1} Z_{i}^{\mathfrak{q}},\left[\bar{X}_{0}^{\mathfrak{q}}, Z_{i}^{\mathfrak{q}}\right]=0, \quad\left[X_{0}^{\mathfrak{q}}, Z_{i}^{\mathfrak{q}}\right]=-X_{i}^{\mathfrak{h}},} \\
& {\left[Z_{0}^{\mathfrak{h}}, X_{i}^{\mathfrak{h}}\right]=\sqrt{-1} X_{i}^{\mathfrak{h}}, \quad\left[\bar{X}_{0}^{\mathfrak{q}}, X_{i}^{\mathfrak{h}}\right]=-Z_{i}^{\mathfrak{q}}, \quad\left[X_{0}^{\mathfrak{q}}, X_{i}^{\mathfrak{h}}\right]=0,} \\
& {\left[Z_{0}^{\mathfrak{h}}, X_{i j}^{\mathfrak{h}}\right]=0, \quad\left[\bar{X}_{0}^{\mathfrak{q}}, X_{i j}^{\mathfrak{h}}\right]=0, \quad\left[X_{0}^{\mathfrak{q}}, X_{i j}^{\mathfrak{h}}\right]=0 .}
\end{aligned}
$$


Consider the one parameter subgroup

$$
a_{t}=\exp \left(t Y_{0}\right)=\operatorname{diag}\left(1_{p-1},\left[\begin{array}{l}
\cosh t \sinh t \\
\sinh t
\end{array} \cosh t\right], 1_{q-1}\right), \quad(t \in \mathbb{R})
$$

of $G$. Then by general theory, the group $G$ is a disjoint union of double cosets $H a_{t} K(t \geqslant 0)$ and the Lie algebra $\mathfrak{g}=\operatorname{Ad}\left(a_{t}\right)^{-1} \mathfrak{h}+\mathfrak{a}+\mathfrak{k}$ if $t>0$. By direct computation, we have

$$
\begin{aligned}
& X_{0}^{\mathfrak{q}}=\frac{1}{2} Y_{0}-\frac{1}{2} \frac{\sqrt{-1}}{\sinh (2 t)} \operatorname{Ad}\left(a_{t}\right)^{-1} Z_{0}^{\mathfrak{h}}+\frac{\sqrt{-1}}{2} \frac{\cosh (2 t)}{\sinh (2 t)} Z_{0}^{\mathfrak{h}}, \\
& X_{j}^{\mathfrak{q}}=\frac{1}{\sinh t} \operatorname{Ad}\left(a_{t}\right)^{-1} Z_{j}^{\mathfrak{h}}-\frac{\cosh t}{\sinh t} Z_{j}^{\mathfrak{h}}, \quad(1 \leqslant j \leqslant q-1), \\
& X_{i}^{\mathfrak{h}}=\frac{1}{\cosh t} \operatorname{Ad}\left(a_{t}\right)^{-1} X_{i}^{\mathfrak{h}}-\frac{\sinh t}{\cosh t} Z_{i}^{\mathfrak{q}}, \quad(1 \leqslant i \leqslant p-1) .
\end{aligned}
$$

Lemma 1. For $1 \leqslant i, \alpha \leqslant p-1$ and $1 \leqslant j, \beta \leqslant q-1$ and $t>0$, the following hold in $U\left(\mathfrak{g}_{\mathbb{C}}\right)$ modulo $\left(\operatorname{Ad}\left(a_{t}\right)^{-1} \mathfrak{h}_{\mathbb{C}}\right) U\left(\mathfrak{g}_{\mathbb{C}}\right)$ :

$$
\begin{aligned}
X_{0}^{\mathfrak{q}} \bar{X}_{0}^{\mathfrak{q}} & \equiv \frac{1}{4} Y_{0}^{2}+\frac{1}{4}(\tanh t+\operatorname{coth} t) Y_{0}+\frac{1}{16}(\tanh t+\operatorname{coth} t)^{2}\left(Z_{0}^{\mathfrak{h}}\right)^{2}-\frac{\sqrt{-1}}{2} Z_{0}^{\mathfrak{h}}, \\
X_{0}^{\mathfrak{q}} \bar{X}_{i}^{\mathfrak{h}} & \equiv-\frac{\tanh t}{2} Y_{0} \bar{Z}_{i}^{\mathfrak{q}}-\frac{\sqrt{-1}}{4}\left(1+\tanh ^{2} t\right) Z_{0}^{\mathfrak{h}} \bar{Z}_{i}^{\mathfrak{q}}-\bar{Z}_{i}^{\mathfrak{q}}, \\
X_{i}^{\mathfrak{h}} \bar{X}_{0}^{\mathfrak{q}} & \equiv-\frac{\tanh t}{2} Y_{0} Z_{i}^{\mathfrak{q}}+\frac{\sqrt{-1}}{4}\left(1+\tanh ^{2} t\right) Z_{0}^{\mathfrak{h}} Z_{i}^{\mathfrak{q}}, \\
X_{0}^{\mathfrak{q}} \bar{X}_{j}^{\mathfrak{q}} & \equiv-\frac{\operatorname{coth} t}{2} Y_{0} \bar{Z}_{j}^{\mathfrak{h}}-\frac{\sqrt{-1}}{4}\left(1+\operatorname{coth}^{2} t\right) Z_{0}^{\mathfrak{h}} \bar{Z}_{j}^{\mathfrak{h}}-\bar{Z}_{j}^{\mathfrak{h}}, \\
X_{j}^{\mathfrak{q}} \bar{X}_{0}^{\mathfrak{q}} & \equiv-\frac{\operatorname{coth} t}{2} Y_{0} Z_{j}^{\mathfrak{h}}+\frac{\sqrt{-1}}{4}\left(1+\operatorname{coth}^{2} t\right) Z_{0}^{\mathfrak{h}} Z_{j}^{\mathfrak{h}}, \\
X_{i}^{\mathfrak{h}} \bar{X}_{\alpha}^{\mathfrak{h}} & \equiv \delta_{i \alpha} \frac{\tanh t}{2} Y_{0}+\tanh ^{2} t \bar{Z}_{\alpha}^{\mathfrak{q}} Z_{i}^{\mathfrak{q}}-\frac{\sqrt{-1}}{4}\left(1+\tanh ^{2} t\right) \delta_{i \alpha} Z_{0}^{\mathfrak{h}}, \\
X_{j}^{\mathfrak{q}} \bar{X}_{\beta}^{\mathfrak{q}} & \equiv \delta_{j \beta} \frac{\operatorname{coth} t}{2} Y_{0}+\operatorname{coth}^{2} t \bar{Z}_{\beta}^{\mathfrak{h}} Z_{j}^{\mathfrak{h}}-\frac{\sqrt{-1}}{4}\left(1+\operatorname{coth}^{2} t\right) \delta_{j \beta} Z_{0}^{\mathfrak{h}}, \\
X_{j}^{\mathfrak{q}} \bar{X}_{i}^{\mathfrak{h}} & \equiv \bar{Z}_{i}^{\mathfrak{q}} Z_{j}^{\mathfrak{h}}, \\
X_{i}^{\mathfrak{h}} \bar{X}_{j}^{\mathfrak{q}} & \equiv \bar{Z}_{j}^{\mathfrak{h}} Z_{i}^{\mathfrak{q}} .
\end{aligned}
$$

Proof. By using the formulas (2.2), we prove this lemma in a similar way to [26, Lemma 7.1.2].

The definitions and formulas of this section are used in $\S 4$.

2.4. Invariant measures. Let $\mathrm{d} k$ and $\mathrm{d} k_{0}$ be the Haar measures of the compact groups $K$ and $H \cap K$ with total volume 1 respectively. Then we can take a unique Haar measure $\mathrm{d} g$ (resp. $\mathrm{d} h)$ of $G($ resp. $H)$ such that the quotient measure $\frac{\mathrm{d} g}{\mathrm{~d} k}$ (resp. $\frac{\mathrm{d} h}{\mathrm{~d} k_{0}}$ ) coincides with the invariant measure on the symmetric space $G / K$ (resp. $H / K_{H}$ ) determined by the Kähler volume form. 
Lemma 2. For any integrable function $f$ on $G$, we have

$$
\int_{G} f(g) \mathrm{d} g=\int_{H} \mathrm{~d} h \int_{K} \mathrm{~d} k \int_{0}^{+\infty} f\left(h a_{t} k\right) \varrho(t) \mathrm{d} t
$$

with $\mathrm{d} t$ the Lebesgue measure of $\mathbb{R}$ and

$$
\varrho(t)=\frac{2 \pi^{q}}{\Gamma(q)}(\sinh t)^{2 q-1}(\cosh t)^{2 p-1} .
$$

Proof. Similar to [32, Lemma 4.1].

\section{Certain invariant tensors}

For a $C^{\infty}$-manifold $U$, let $\mathcal{A}(U)$ denote the space of $C^{\infty}$-differential forms on $U$ and $\mathcal{A}_{\mathrm{c}}(U)$ the subspace of those forms with compact support; when necessary we topologize these spaces in the usual way. When $U$ has a complex structure, $\mathcal{A}^{a, b}(U)$ denotes the space of $C^{\infty}$-differential forms of bidegree $(a, b)$.

In this section, for $U=G / K$ we define some element dual to $\mathcal{A}_{\mathrm{c}}(U)$ by the $H$-orbit in $U$. Some of the contents of this section may be not found in the literature.

3.1. A current defined by the symmetric subgroup. Let $j: H / H \cap K \hookrightarrow$ $G / K$ be the natural inclusion. Then a $(q, q)$-current $\delta_{H / H \cap K}$ on $G / K$ is defined by the integration

$$
\left\langle\delta_{H / H \cap K}, \alpha\right\rangle=\int_{H / H \cap K} j^{*} \alpha, \quad \alpha \in \mathcal{A}_{\mathrm{c}}(G / K) .
$$

Lemma 3. For $\alpha \in \mathcal{A}_{\mathrm{c}}(G / K)$, we have

$$
\left\langle\Lambda^{q-d} \delta_{H / H \cap K}, * \bar{\alpha}\right\rangle=\int_{H}\left(\Lambda^{q-d}\left(* \operatorname{vol}_{\mathfrak{p} \cap \mathfrak{h}}\right) \mid \alpha(h)\right) \mathrm{d} h .
$$

Here

$$
\operatorname{vol}_{\mathfrak{p} \cap \mathfrak{h}}=\frac{1}{(q(p-1)) !} \omega_{\mathfrak{p} \cap \mathfrak{h}}^{q(p-1)} \in \bigwedge^{(p-1) q,(p-1) q} \mathfrak{p}_{\mathbb{C}}^{*}
$$

is the $K \cap H$-invariant tensor corresponding to the Kähler volume form of $H / H \cap K$.

Proof. We may assume the bidegree of $\alpha$ is $(d, d)$. Let $v_{H / H \cap K}$ be the Kähler volume form of $H / H \cap K$ and $*_{H}$ the Hodge star operator of $H / H \cap K$. Then the 0 form $\left\{*_{H} j^{*}\left(\Lambda^{q-d_{*}} \bar{\alpha}\right)\right\}$ on $H / H \cap K$ corresponds to the function $\left(\Lambda^{q-d}(* \bar{\alpha}(h)) \mid \operatorname{vol}_{\mathfrak{p} \cap \mathfrak{h}}\right)$ 
on $H$. By this remark, we compute

$$
\begin{aligned}
\left\langle\Lambda^{q-d} \delta_{H / H \cap K}, * \bar{\alpha}\right\rangle & =\left\langle\delta_{H / H \cap K}, \Lambda^{q-d}(* \bar{\alpha})\right\rangle \\
& =\int_{H / H \cap K}\left(\Lambda^{q-d}(* \bar{\alpha}(h)) \mid \operatorname{vol}_{\mathfrak{p} \cap \mathfrak{h}}\right) \mathrm{d} h \\
& =\int_{H / H \cap K}\left(\operatorname{vol}_{\mathfrak{p} \cap \mathfrak{h} \mid} \mid \Lambda^{q-d}(* \alpha(h))\right) \mathrm{d} h \\
& =\int_{H / H \cap K}\left(\Lambda^{q-d}\left(* \operatorname{vol}_{\mathfrak{p} \cap \mathfrak{h}}\right) \mid \alpha(h)\right) \mathrm{d} h .
\end{aligned}
$$

3.2. $K$-spectrum of a certain cyclic $K$-module. For our purpose, it is important to understand the nature of the tensor $\Lambda^{q-d}\left(* \operatorname{vol}_{\mathfrak{p} \cap \mathfrak{h}}\right)$ in some detail. The aim of this subsection is to obtain an $\Omega_{\mathfrak{k}}$-eigendecomposition of the tensor $\Lambda^{q-d}\left(* \operatorname{vol}_{\mathfrak{p} \cap \mathfrak{h}}\right)$. Here $\Omega_{\mathfrak{k}}$ is the Casimir element of $K$ corresponding to the invariant form $B_{\mathfrak{g}}$. For the construction of the secondary spherical function in $\S 5$, we need the decomposition of $\Lambda^{q-d}\left(* \operatorname{vol}_{\mathfrak{p} \cap \mathfrak{h}}\right)$ given in Proposition 11.

The coadjoint representation of $K$ on $\mathfrak{p}_{\mathbb{C}}^{*}$ is naturally extended to a unitary representation $\tau: K \rightarrow \operatorname{GL}\left(\wedge \mathfrak{p}_{\mathbb{C}}^{*}\right)$ in such a way that $\tau(k)(\alpha \wedge \beta)=\tau(k) \alpha \wedge$ $\tau(k) \beta$ holds for $\alpha, \beta \in \Lambda \mathfrak{p}_{\mathbb{C}}^{*}$ and $k \in K$. For $(a, b) \in \mathbb{N}^{2}, \tau^{a, b}$ denotes the subrepresentation of $\tau$ on $\bigwedge^{a, b} \mathfrak{p}_{\mathbb{C}}^{*}$.

For $1 \leqslant i \leqslant p, 1 \leqslant j \leqslant q$, let us define $\omega_{i j} \in \mathfrak{p}_{\mathbb{C}}^{*}$ by $\omega_{i j}\left(E_{\alpha, p+\beta}\right)=\delta_{i \alpha} \delta_{j \beta}(1 \leqslant$ $\alpha \leqslant p, 1 \leqslant \beta \leqslant q), \omega_{i j} \mid \mathfrak{p}_{-}=0$. Then $\omega_{i j}$ 's and their complex conjugates $\bar{\omega}_{i j}$ comprise a $\mathbb{C}$-basis of $\mathfrak{p}_{\mathbb{C}}^{*}$ dual to the basis of matrix units in $\mathfrak{p}_{\mathbb{C}}$.

Lemma 4. (1) The family $2^{-1}\left(\omega_{i j}+\bar{\omega}_{i j}\right), 2^{-1} \sqrt{-1}\left(\bar{\omega}_{i j}-\omega_{i j}\right),(1 \leqslant i \leqslant p, 1 \leqslant$ $j \leqslant q)$ is an orthonormal basis of $\mathfrak{p}^{*}$ with respect to $B_{\mathfrak{p}^{*}}$ and is dual to the orthonormal basis $E_{i, p+j}+E_{p+j, i}, \sqrt{-1}\left(E_{i, p+j}-E_{p+j, i}\right)$ of $\mathfrak{p}$.

(2) We have $\left(\omega_{i j} \mid \omega_{\alpha \beta}\right)=\left(\bar{\omega}_{i j} \mid \bar{\omega}_{\alpha \beta}\right)=2 \delta_{i \alpha} \delta_{j \beta}$ and $\left(\omega_{i j} \mid \bar{\omega}_{\alpha \beta}\right)=0$.

(3) The action of the matrix units in $\mathfrak{k}_{\mathbb{C}}$ on $\omega_{i j}(1 \leqslant i \leqslant p, 1 \leqslant j \leqslant q)$ is given by

$$
\begin{aligned}
\tau\left(E_{\mu, \alpha}\right) \omega_{i j} & =-\delta_{i \mu} \omega_{\alpha j}, \quad \tau\left(E_{\mu, \alpha}\right) \bar{\omega}_{i j}=\delta_{i \alpha} \bar{\omega}_{\mu j}, \quad(1 \leqslant \mu, \alpha \leqslant p), \\
\tau\left(E_{p+\nu, p+\beta}\right) \omega_{i j} & =\delta_{j \beta} \omega_{i \nu}, \quad \tau\left(E_{p+\nu, p+\beta}\right) \bar{\omega}_{i j}=-\delta_{j \nu} \bar{\omega}_{i \beta}, \quad(1 \leqslant \nu, \beta \leqslant q) .
\end{aligned}
$$

Proof. Direct computation. 
For $1 \leqslant \mu, \alpha \leqslant p$, set

$$
\gamma_{\mu \alpha}=\frac{\sqrt{-1}}{2} \sum_{j=1}^{q} \omega_{\mu j} \wedge \bar{\omega}_{\alpha j} \in \bigwedge^{1,1} \mathfrak{p}_{\mathbb{C}}^{*} .
$$

We have a concise expression of Kähler forms in terms of $\gamma_{\mu \alpha}$ :

Lemma 5. We have

$$
\omega_{\mathfrak{p}}=\sum_{\mu=1}^{p} \gamma_{\mu \mu}, \quad \omega_{\mathfrak{p} \cap \mathfrak{h}}=\omega_{\mathfrak{p}}-\gamma_{p p} .
$$

The tensor $\gamma_{p p}$ is $H \cap K$-invariant.

Proof. Direct consequence of definitions.

The action of $\mathfrak{k}_{\mathbb{C}}$ on the tensors $\gamma_{\mu \alpha}$ is given as follows.

Lemma 6. For $1 \leqslant i, \alpha, \mu \leqslant p-1$, we have

$$
\begin{array}{rlrl}
\tau\left(Z_{i}^{\mathfrak{q}}\right) \gamma_{p p} & =\gamma_{p i}, & & \tau\left(\bar{Z}_{i}^{\mathfrak{q}}\right) \gamma_{p p}=\gamma_{i p}, \\
\tau\left(Z_{i}^{\mathfrak{q}}\right) \gamma_{\alpha p} & =-\delta_{i \alpha} \gamma_{p p}+\gamma_{\alpha i}, & & \tau\left(\bar{Z}_{i}^{\mathfrak{q}}\right) \gamma_{p \alpha}=-\delta_{i \alpha} \gamma_{p p}+\gamma_{i \alpha}, \\
\tau\left(Z_{i}^{\mathfrak{q}}\right) \gamma_{p \alpha}=0, & & \tau\left(\bar{Z}_{i}^{\mathfrak{q}}\right) \gamma_{\alpha p}=0, \\
\tau\left(Z_{i}^{\mathfrak{q}}\right) \gamma_{\alpha \mu} & =-\delta_{i \alpha} \gamma_{p \mu}, & & \tau\left(\bar{Z}_{i}^{\mathfrak{q}}\right) \gamma_{\alpha \mu}=-\delta_{i \mu} \gamma_{\alpha p} .
\end{array}
$$

For $1 \leqslant j, \beta \leqslant q-1,1 \leqslant \mu, \alpha \leqslant p$, we have

$$
\begin{aligned}
& \tau\left(Z_{j}^{\mathfrak{h}}\right) \gamma_{\mu \alpha}=\tau\left(\bar{Z}_{j}^{\mathfrak{h}}\right) \gamma_{\mu \alpha}=0, \\
& \tau\left(E_{p+j+1, p+\beta+1}\right) \gamma_{\mu \alpha}=\tau\left(E_{p+1, p+1}\right) \gamma_{\mu \alpha}=0 .
\end{aligned}
$$

For $1 \leqslant i, \alpha \leqslant p, 1 \leqslant \mu, \lambda \leqslant p$, we have

$$
\tau\left(E_{i, \alpha}\right) \gamma_{\mu \lambda}=-\delta_{i \mu} \gamma_{\alpha \lambda}+\delta_{\alpha \lambda} \gamma_{\mu i} .
$$

Proof. This follows from Lemma 4 readily.

For $\gamma \in \bigwedge^{1,1} \mathfrak{p}_{\mathbb{C}}^{*}$, the $r$-fold wedge product $\gamma \wedge \gamma \wedge \cdots \wedge \gamma$ is denoted by $\gamma^{r}$.

Lemma 7. For $0 \leqslant d \leqslant q$,

$$
\Lambda^{q-d}\left(* \operatorname{vol}_{\mathfrak{p} \cap \mathfrak{h}}\right)=\frac{(q-d) !}{d !} \gamma_{p p}^{d}
$$


Proof. For any subset $M$ of $\{(i, j) \mid 1 \leqslant i \leqslant p, 1 \leqslant j \leqslant q\}$, set $w_{M}=\prod_{(i, j) \in M} \omega_{i j} \wedge$ $\bar{\omega}_{i j}$. Then from $[38$, p.20,p.21], we have

$$
\begin{aligned}
* w_{M} & =\left(\frac{2}{\sqrt{-1}}\right)^{2 \nu(M)-p q} w_{M^{\prime}}, \\
\Lambda\left(w_{M}\right) & =\frac{2}{\sqrt{-1}} \sum_{\mu \in M} w_{M-\{\mu\}}
\end{aligned}
$$

with $\nu(M)$ the cardinality of $M$, and $M^{\prime}$ the complement of $M$.

If suffices to show

$$
\begin{aligned}
* \operatorname{vol}_{\mathfrak{p} \cap \mathfrak{h}} & =\frac{1}{q !} \gamma_{p p}^{q}, \\
\Lambda\left(\gamma_{p p}^{d}\right) & =d(q-d+1) \gamma_{p p}^{d-1}, \quad(1 \leqslant d \leqslant q) .
\end{aligned}
$$

Since

$$
\operatorname{vol}_{\mathfrak{p} \cap \mathfrak{h}}=\left(\frac{\sqrt{-1}}{2}\right)^{p q-q} \prod_{i=1}^{p-1} \prod_{j=1}^{q} \omega_{i j} \wedge \bar{\omega}_{i j}, \quad \gamma_{p p}^{q}=q ! \prod_{j=1}^{q} \omega_{p j} \wedge \bar{\omega}_{p j},
$$

by (3.1), we compute

$$
* \operatorname{vol}_{\mathfrak{p} \cap \mathfrak{h}}=\left(\frac{\sqrt{-1}}{2}\right)^{p q-q} *\left(\prod_{i=1}^{p-1} \prod_{j=1}^{q} \omega_{i j} \wedge \bar{\omega}_{i j}\right)=\left(\frac{\sqrt{-1}}{2}\right)^{q} \prod_{j=1}^{q} \omega_{p j} \wedge \bar{\omega}_{p j}=\frac{1}{q !} \gamma_{p p}^{q} .
$$

This completes the proof of (3.3). Let $S_{d}$ be the set of all $M \subset\{(p, j) \mid 1 \leqslant j \leqslant q\}$ with $\nu(M)=d$. Since $\gamma_{p p}^{d}=\left(\frac{\sqrt{-1}}{2}\right)^{d} d ! \sum_{M \in S_{d}} w_{M}$, by (3.2), we compute

$$
\begin{aligned}
\Lambda\left(\gamma_{p p}^{d}\right) & =\left(\frac{\sqrt{-1}}{2}\right)^{d} d ! \sum_{M \in S_{d}} \frac{2}{\sqrt{-1}} \sum_{\mu \in M} w_{M-\{\mu\}} \\
& =\left(\frac{\sqrt{-1}}{2}\right)^{d-1} d !(q-d+1) \sum_{N \in S_{d-1}} w_{N}=d(q-d+1) \gamma_{p p}^{d-1}
\end{aligned}
$$

to have (3.4).

In order to have a decomposition of $\gamma_{p p}^{d}$ into eigenvectors of the Casimir operator $\Omega_{\mathfrak{k}}$, we first analyze the $K$-spectrum of $U\left(\mathfrak{k}_{\mathbb{C}}\right) \gamma_{p p}^{d}$, the cyclic $U\left(\mathfrak{k}_{\mathbb{C}}\right)$-submodule of $\bigwedge^{d, d} \mathfrak{p}_{\mathbb{C}}^{*}$ generated by $\gamma_{p p}^{d}$.

Since $\mathfrak{k}_{\mathbb{C}} \cong \mathfrak{g l}_{p}(\mathbb{C}) \oplus \mathfrak{g l}_{q}(\mathbb{C})$, the highest weight of an irreducible representation of $\mathfrak{k}$ is supposed to take the form

$$
\lambda=\left[l_{1}, l_{2}, \ldots, l_{p}\right] \oplus\left[m_{1}, m_{2}, \ldots, m_{q}\right]
$$


with $l_{i}, m_{j} \in \mathbb{Z}$ such that $l_{1} \geqslant l_{2} \geqslant \cdots \geqslant l_{p}, m_{1} \geqslant m_{2} \geqslant \cdots m_{q}$.

Lemma 8. Let $0 \leqslant d \leqslant q$ and $V$ an irreducible $K$-submodule of $U\left(\mathfrak{k}_{\mathbb{C}}\right) \gamma_{p p}^{d}$. Then the highest weight of $V$ is of the form $[\kappa, 0, \ldots, 0,-\kappa] \oplus[0, \ldots, 0]$ with an integer $0 \leqslant \kappa \leqslant d$.

Proof. Let us fix a $K$-invariant inner product on $U\left(\mathfrak{k}_{\mathbb{C}}\right) \gamma_{p p}^{d}$ and take the orthogonal complement $V^{\perp}$ of $V$ in $U\left(\mathfrak{k}_{\mathbb{C}}\right) \gamma_{p p}^{d}$. Since the projector pr $: U\left(\mathfrak{k}_{\mathbb{C}}\right) \gamma_{p p}^{d} \rightarrow V$ associated with the decomposition $\mathrm{U}\left(\mathfrak{k}_{\mathbb{C}}\right) \gamma_{p p}^{d}=V \oplus V^{\perp}$ is a surjective $K$-homomorphism and since $\gamma_{p p}^{d}$ is $H \cap K$-invariant, the vector $v=\operatorname{pr}\left(\gamma_{p p}^{d}\right) \in V$ yields an $H \cap K$ invariant $U\left(\mathfrak{k}_{\mathbb{C}}\right)$-cyclic vector of $V$. In particular, $v \neq 0$ and $V^{H \cap K} \neq\{0\}$. Let $\lambda$ be the highest weight of $V$, which is supposed to have the form (3.5). Since $\mathfrak{k}_{\mathbb{C}} \cap \mathfrak{h}_{\mathbb{C}}=\left(\mathfrak{g l}_{p-1}(\mathbb{C}) \oplus \mathbb{C}\right) \oplus \mathfrak{g l}_{q}(\mathbb{C})$, the condition $V^{H \cap K} \neq\{0\}$ yields that the irreducible $\mathfrak{g l}_{p}(\mathbb{C})$-module of highest weight $\left[l_{1}, \ldots, l_{p}\right]$ contains the trivial representation of $\mathfrak{g l}_{p-1}(\mathbb{C})$ and that the irreducible $\mathfrak{g l}_{q}(\mathbb{C})$-module with highest weight $\left[m_{1}, \ldots, m_{q}\right]$ is trivial. Hence

$$
l_{i}=0(\forall i \in\{2, \ldots, p-1\}), \quad m_{j}=0(\forall j \in\{1, \ldots, q\})
$$

by the $\mathfrak{g l}_{p-1} \rightarrow \mathfrak{g l}_{p}$ branching law ([6, Theorem 8.1.1 (p.350)]). The center of $K$ acts on $V$ trivially because a central element of $K$ fixes the tensor $\gamma_{p p}^{d}$. Hence the sum $l_{1}+\cdots+l_{p}$ should be zero. This, combined with the condition (3.6), forces that $\lambda=[\kappa, 0 \ldots,-\kappa] \oplus[0, \ldots, 0]$ with some $\kappa \in \mathbb{N}$. It remains to show $0 \leqslant \kappa \leqslant d$. For that, we examine the $T$-weights occurring in $V$, where $T=$ $\left\{\operatorname{diag}\left(t_{1}, \ldots, t_{p+q}\right) \mid t_{i} \in \mathrm{U}(1)\right\}$. For $1 \leqslant i \leqslant p+q$, let $\epsilon_{i}: T \rightarrow \mathrm{U}(1)$ be the character defined by $\epsilon_{i}\left(\operatorname{diag}\left(t_{1}, \ldots, t_{p+q}\right)\right)=t_{i}$. From Lemma 6 , the $T$-weight of the element $\gamma_{\mu \alpha}(1 \leqslant \mu, \alpha \leqslant p)$ equals $\epsilon_{\alpha}-\epsilon_{\mu}$. Lemma 6 also shows that $\mathrm{U}\left(\mathfrak{k}_{\mathbb{C}}\right) \gamma_{p p}^{d}$ is contained in $\mathbb{C}[\Gamma]^{(d)}$, the subspace of $\Lambda \mathfrak{p}_{\mathbb{C}}^{*}$ spanned by the products of $d$ of $p^{2}$ tensors $\gamma_{\mu \alpha}(1 \leqslant \mu, \alpha \leqslant p)$. In particular the highest weight $\lambda=\kappa\left(\epsilon_{1}-\epsilon_{p}\right)$ of $V$ is one of $T$-weights occurring in $\mathbb{C}[\Gamma]^{(d)}$. It is obvious that a $T$-weight of $\mathbb{C}[\Gamma]^{(d)}$, especially $\kappa\left(\epsilon_{1}-\epsilon_{p}\right)$, is a sum of $d$ weights of the form $\epsilon_{\mu}-\epsilon_{\alpha}(1 \leqslant \mu, \alpha \leqslant p)$. This implies $0 \leqslant \kappa \leqslant d$.

For $0 \leqslant \kappa \leqslant d$, let $V_{\kappa}^{(d)}$ be the $[\kappa, 0, \ldots, 0,-\kappa] \oplus[0, \ldots, 0]$-isotypic part of $U\left(\mathfrak{k}_{\mathbb{C}}\right) \gamma_{p p}^{d}$. Then Lemma 8 implies

$$
U\left(\mathfrak{k}_{\mathbb{C}}\right) \gamma_{p p}^{d}=\bigoplus_{\kappa=0}^{d} V_{\kappa}^{(d)} .
$$


Note that $V_{0}^{(d)}$ is a trivial representation of $K$.

Lemma 9. For $0 \leqslant d \leqslant q$, the $K$-module $V_{d}^{(d)}$ is irreducible.

Proof. We have $\tau\left(\bar{Z}_{1}^{\mathfrak{q}}\right)^{d} \gamma_{p p}^{d}=d ! \gamma_{1 p}^{d}$ by a short computation using formulas in Lemma 6. Hence the tensor $\gamma_{1 p}^{d}$ belongs to $U\left(\mathfrak{k}_{\mathbb{C}}\right) \gamma_{p p}^{d}$. Let $\mathfrak{u}$ be the nilpotent subalgebra of $\mathfrak{g l}_{p+q}(\mathbb{C})$ formed by all the lower triangular nilpotent matrices. Then by the formulas in Lemma 6 , it is easy to see that $\gamma_{1 p}^{d}$ is annihilated by all the matrix units $E_{\alpha \beta} \in \mathfrak{k}_{\mathbb{C}} \cap \mathfrak{u}$. This proves that $\gamma_{1 p}^{d}$ is an extremal vector of $\mathfrak{k}_{\mathbb{C}}$ lying in $U\left(\mathfrak{k}_{\mathbb{C}}\right) \gamma_{p p}^{d}$, which generates an irreducible $K$-module of lowest weight $-d\left(\epsilon_{1}-\epsilon_{p}\right)$. Therefore $V_{d}^{(d)} \neq\{0\}$. It is easy to show that the $d\left(\epsilon_{p}-\epsilon_{1}\right)$ weight space of $\mathbb{C}[\Gamma]^{(d)}$ coincides with the one dimensional space $\mathbb{C} \gamma_{1 p}^{d}$. This implies $d\left(\epsilon_{p}-\epsilon_{1}\right)$-weight space of $V_{d}^{(d)}$ also coincides with $\mathbb{C} \gamma_{1 p}^{d}$. Hence $V_{d}^{(d)}$ is irreducible.

Corollary 10. Let $0 \leqslant d \leqslant q$. Then the operator $\prod_{\kappa=0}^{d}\left(-4^{-1} \Omega_{\mathfrak{k}}+\kappa(\kappa+p-1)\right)$ annihilates the tensor $\gamma_{p p}^{d}$ :

$$
\prod_{\kappa=0}^{d}\left(-4^{-1} \Omega_{\mathfrak{k}}+\kappa(\kappa+p-1)\right) \gamma_{p p}^{d}=0 .
$$

Proof. Write the element $\gamma_{p p}^{d}$ as the sum

$$
\gamma_{p p}^{d}=\sum_{\kappa=0}^{d} v_{\kappa}, \quad v_{\kappa} \in V_{\kappa}^{(d)}
$$

along the decomposition (3.7). Since the eigenvalue of $\Omega_{\mathfrak{k}}$ on an irreducible $K$ module of highest weight $[\kappa, 0, \ldots, 0,-\kappa] \oplus[0, \ldots, 0]$ is $4 \kappa(\kappa+p-1)$, the element $v_{\kappa}$ is annihilated by $-4^{-1} \Omega_{\mathfrak{k}}+\kappa(\kappa+p-1)$, a factor of $\prod_{\kappa=0}^{d}\left(-4^{-1} \Omega_{\mathfrak{k}}+\kappa(\kappa+p-1)\right)$. The conclusion follows from this remark and the decomposition (3.8).

For $0 \leqslant d \leqslant q, 0 \leqslant \kappa \leqslant d$, set

$$
\theta_{\kappa}^{(d)}=\frac{(q-d) !}{d !} \prod_{\substack{0 \leqslant \alpha \leqslant d \\ \alpha \neq \kappa}} \frac{-4^{-1} \Omega_{\mathfrak{k}}+\alpha(\alpha+p-1)}{(\alpha-\kappa)(\alpha+\kappa+p-1)} \cdot \gamma_{p p}^{d} \in \bigwedge^{d, d} \mathfrak{p}_{\mathbb{C}}^{*}
$$

Proposition 11. Let $0 \leqslant d \leqslant q$. 
(1) For each $0 \leqslant \kappa \leqslant d$, the tensor $\theta_{\kappa}^{(d)}$ is a nonzero eigenvector of $\Omega_{\mathfrak{k}}$ with the eigenvalue $4 \kappa(\kappa+p-1)$, i.e.,

$$
\Omega_{\mathfrak{k}} \theta_{\kappa}^{(d)}=4 \kappa(\kappa+p-1) \theta_{\kappa}^{(d)}, \quad \theta_{\kappa}^{(d)} \neq 0 .
$$

The tensor $\theta_{\kappa}^{(d)}$ is $H \cap K$-invariant and is a $U\left(\mathfrak{k}_{\mathbb{C}}\right)$-cyclic vector of $V_{\kappa}^{(d)}$.

(2) We have

$$
\Lambda^{q-d}\left(* \operatorname{vol}_{\mathfrak{p} \cap \mathfrak{h}}\right)=\sum_{\kappa=0}^{d} \theta_{\kappa}^{(d)} .
$$

Moreover, the tensors $\theta_{d}^{(d)}(0 \leqslant d \leqslant q)$ are primitive, i.e., $\Lambda \theta_{d}^{(d)}=0$; we have $\Lambda \theta_{\kappa}^{(d)}=\theta_{\kappa}^{(d-1)}(0 \leqslant \kappa<d)$.

Proof. Let $T$ be an indeterminate and consider the polynomial $F_{d}(T)=\prod_{\kappa=0}^{d}(T+$ $\kappa(\kappa+p-1))$. The $d+1$ integers $\alpha_{\kappa}=-\kappa(\kappa+p-1)(0 \leqslant \kappa \leqslant d)$ are mutually distinct and coincides with the set of roots of $F_{d}(T)$. Hence the formula $\frac{1}{F_{d}(T)}=$ $\sum_{\kappa=0}^{d} \frac{1}{F_{d}^{\prime}\left(\alpha_{\kappa}\right)\left(T-\alpha_{\kappa}\right)}$, or equivalently

$$
1=\sum_{\kappa=0}^{d} F_{d}^{\prime}\left(\alpha_{\kappa}\right)^{-1} F_{d, \kappa}(T)
$$

holds, where $F_{d}(T)=\left(T-\alpha_{\kappa}\right) F_{d, \kappa}(T)$. A computation shows

$$
\theta_{\kappa}^{(d)}=F_{d}^{\prime}\left(\alpha_{\kappa}\right)^{-1} F_{d, \kappa}\left(-4^{-1} \Omega_{\mathfrak{k}}\right) \frac{(q-d) !}{d !} \gamma_{p p}^{d} .
$$

The substitution $T=-4^{-1} \Omega_{\mathfrak{k}}$ in the identity (3.11) yields yet another identity of operators on $\Lambda \mathfrak{p}_{\mathbb{C}}^{*}$; apply this to the element $\Lambda^{q-d}\left(* \operatorname{vol}_{\mathfrak{p} \cap \mathfrak{h}}\right)$. Then we obtain the identity (3.10) by (3.12) and Lemma 7 .

Since $(T+\kappa(\kappa+p-1)) F_{d, \kappa}(T)=F_{d}(T)$, we compute

$$
\left\{-4^{-1} \Omega_{\mathfrak{k}}+\kappa(\kappa+p-1)\right\} \theta_{\kappa}^{(d)}=F_{d}^{\prime}\left(\alpha_{\kappa}\right)^{-1} F_{d}\left(-4^{-1} \Omega_{\mathfrak{k}}\right) \frac{(q-d) !}{d !} \gamma_{p p}^{d}=0
$$

using Corollary 10 to prove the second equality. This shows the second statement of (1). Since $\gamma_{p p}^{d}$ is $H \cap K$-invariant (Lemma 5), the defining formula (3.12) shows the $H \cap K$-invariance of $\theta_{\kappa}^{(d)}$.

Lemma 7 implies

$$
\Lambda\left(\frac{(q-d) !}{d !} \gamma_{p p}^{d}\right)=\frac{(q-d+1) !}{(d-1) !} \gamma_{p p}^{d-1}
$$


Let $0 \leqslant \kappa<d$. Then $F_{d}^{\prime}\left(\alpha_{\kappa}\right)=\left(\alpha_{\kappa}+d(d+p-1)\right) F_{d-1}^{\prime}\left(\alpha_{\kappa}\right)$ and $F_{d, \kappa}(T)=$ $(T+d(d+p-1)) F_{d-1, \kappa}(T)$. Using these formulas and noting that $\Lambda$ commutes with $\Omega_{\mathfrak{k}}$, we compute

$$
\begin{aligned}
\Lambda\left(\theta_{\kappa}^{(d)}\right)= & F_{d}^{\prime}\left(\alpha_{\kappa}\right)^{-1} F_{d, \kappa}\left(-4^{-1} \Omega_{\mathfrak{k}}\right) \Lambda\left(\frac{(q-d) !}{d !} \gamma_{p p}^{d}\right) \\
= & F_{d}^{\prime}\left(\alpha_{\kappa}\right)^{-1} F_{d, \kappa}\left(-4^{-1} \Omega_{\mathfrak{k}}\right) \frac{(q-d+1) !}{(d-1) !} \gamma_{p p}^{d-1} \\
= & \left\{\alpha_{\kappa}+d(d+p-1)\right\}^{-1} F_{d-1}^{\prime}\left(\alpha_{\kappa}\right)^{-1} \\
& \times F_{d-1, \kappa}\left(-4^{-1} \Omega_{\mathfrak{k}}\right)\left\{-4^{-1} \Omega_{\mathfrak{k}}+d(d+p-1)\right\} \frac{(q-d+1) !}{(d-1) !} \gamma_{p p}^{d-1} \\
= & \left\{\alpha_{\kappa}+d(d+p-1)\right\}^{-1}\left\{-4^{-1} \Omega_{\mathfrak{k}}+d(d+p-1)\right\} \theta_{\kappa}^{(d-1)} \\
= & \theta_{\kappa}^{(d-1)} .
\end{aligned}
$$

Note we use $\Omega_{\mathfrak{k}} \theta_{\kappa}^{(d-1)}=4 \kappa(\kappa+p-1) \theta_{\kappa}^{(d-1)}$ to obtain the last equality. This proves the last statement of (3). In the computation above, the first two equalities (3.13) and (3.14) are true even for $\kappa=d$. Since $F_{d, d}(T)=F_{d-1}(T)$, the right-hand side of (3.14) equals zero by Corollary 10. This proves $\Lambda\left(\theta_{d}^{(d)}\right)=0$.

The element $v_{\kappa}$ in the decomposition (3.8) has to be a $U\left(\mathfrak{k}_{\mathbb{C}}\right)$-cyclic vector of

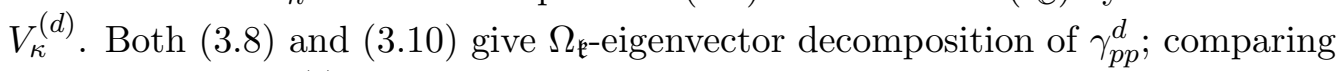
them we obtain $\theta_{\kappa}^{(d)}=\frac{(q-d) !}{d !} v_{\kappa}$ because the relevant eigenvalues $-4 \alpha_{\kappa}$ of $\Omega_{\mathfrak{k}}$ are different for different $\kappa$. Consequently $\theta_{\kappa}^{(d)}$ yields a $U\left(\mathfrak{k}_{\mathbb{C}}\right)$-cyclic vector of $V_{\kappa}^{(d)}$. Since $V_{d}^{(d)} \neq\{0\}$ (Lemma 9), $\theta_{d}^{(d)} \neq 0$. When $\kappa<d$, the formula $\Lambda^{d-\kappa}\left(\theta_{\kappa}^{(d)}\right)=$ $\theta_{\kappa}^{(\kappa)} \neq 0$ shows $\theta_{\kappa}^{(d)} \neq 0$.

Remark: The $\mathfrak{k}$-module $V_{\kappa}^{(d)}$ with $0 \leqslant \kappa<d$ is not necessarily irreducible. For example, when $p=q=2, V_{0}^{(2)}=\mathbb{C} \oplus \mathbb{C}$ is two dimensional and contains a non trivial $K$-invariant tensor orthogonal to $\omega_{\mathfrak{p}}^{2}$.

Example: Consider the case of the rank 2 unitary group $G=\mathrm{U}(p, 2)(p \geqslant 2)$ as an example. We can make the invariant tensors $\theta_{\kappa}^{(2)}$ defined by (3.9) more explicit:

$$
\begin{aligned}
& \theta_{0}^{(2)}=\frac{-1}{2 p(p+1)}(A+2 B-C-E), \\
& \theta_{1}^{(2)}=\frac{1}{p(p+2)}\{A-(p-2) B+(p-1) C+p D-E\} \\
& \theta_{2}^{(2)}=\frac{-1}{2(p+1)(p+2)}\left\{A-2 p B-\left(p^{2}+p+1\right) C+2(p+1) D-E\right\}
\end{aligned}
$$


where

$$
\begin{aligned}
& A=-\sum_{i=1}^{p-1} \sum_{\alpha=1}^{p-1} \gamma_{i \alpha} \wedge \gamma_{\alpha i}, \quad B=-\sum_{i=1}^{p-1} \gamma_{i p} \wedge \gamma_{p i}, \quad C=\gamma_{p p}^{2}, \\
& D=\omega_{\mathfrak{p}} \wedge \gamma_{p p}, \quad E=\omega_{\mathfrak{p}}^{2} .
\end{aligned}
$$

\section{Polar DeComposition of SeVEral Differential operators}

In this subsection we have an expression of several differential operators acting on the space of $H$-invariant forms $\mathcal{A}((G-H K) / K)^{H}$.

4.1. Differential forms. For a right $K$-stable open subset $S$ of $G$ and a unitary representation $(\rho, W)$ of $K$, let $C^{\infty}(S / K ; \rho)$ denote the space of all the $C^{\infty}$ functions $\varphi: S \rightarrow W$ such that

$$
\varphi(g k)=\rho(k)^{-1} \varphi(g), \quad(\forall g \in S, \forall k \in K) .
$$

For $g \in G$, let $L_{g}: x K \mapsto g x K$ be the left translation on $G / K$ by $g$. Its tangent map $T_{o}\left(L_{g}\right)$ at the origin $o=K$ is regarded as a linear map $\mathfrak{p} \rightarrow T_{g K}(G / K)$. Given $\alpha \in \mathcal{A}(S / K)$, a function $\tilde{\alpha} \in C^{\infty}(S / K ; \tau)$ is defined by the formula

$$
\langle\tilde{\alpha}(g), \xi\rangle=\left\langle\alpha(g K),\left\{\wedge T_{o}\left(L_{g}\right)\right\} \xi\right\rangle, \quad(\forall g \in S, \forall \xi \in \bigwedge \mathfrak{p}) .
$$

The map $\alpha \mapsto \tilde{\alpha}$ yields a linear bijection from the space of forms $\mathcal{A}^{a, b}(S / K)$ onto the space of functions $C^{\infty}\left(S / K ; \tau^{a, b}\right)$; we identify these two spaces by this isomorphism.

Since $G-H K$ is a left $H$-stable and right $K$-stable open subset of $G$, both $\mathcal{A}^{a, b}((G-H K) / K)$ and $C^{\infty}\left((G-H K) / K ; \tau^{a, b}\right)$ have natural left actions by $H$, and the isomorphism $\mathcal{A}^{a, b}((G-H K) / K) \cong C^{\infty}\left((G-H K) / K ; \tau^{a, b}\right)$ preserves the $H$-actions.

Lemma 12. Let $\varphi \in C^{\infty}((G-H K) / K ; \tau)^{H}$. Then for each $t>0$, the value $\varphi\left(a_{t}\right)$ belongs to the $M$-invariant part $\left(\bigwedge \mathfrak{p}_{\mathbb{C}}^{*}\right)^{M}$. Conversely, given a $C^{\infty}$-function $\phi$ : $(0,+\infty) \rightarrow\left(\bigwedge \mathfrak{p}_{\mathbb{C}}^{*}\right)^{M}$, there exists a unique function $\varphi \in C^{\infty}((G-H K) / K ; \tau)^{H}$ such that $\varphi\left(a_{t}\right)=\phi(t)(\forall t>0)$.

Proof. Let $t>0$. Since any $m \in M$ commutes with $a_{t}$ and since $M \subset H \cap K$, we have $\varphi\left(a_{t}\right)=\varphi\left(m a_{t} m^{-1}\right)=\tau(m) \varphi\left(a_{t}\right)(\forall m \in M)$, which implies $\phi(t) \in\left(\bigwedge \mathfrak{p}_{\mathbb{C}}^{*}\right)^{M}$. Let us show the converse. Given a $C^{\infty}$-function $\phi:(0,+\infty) \rightarrow\left(\bigwedge \mathfrak{p}_{\mathbb{C}}^{*}\right)^{M}$, we 
define a function $\tilde{\varphi}: H \times(0,+\infty) \times K \rightarrow \bigwedge \mathfrak{p}_{\mathbb{C}}^{*}$ by $\tilde{\varphi}(h, t, k)=\tau(k)^{-1} \phi(t)$. Obviously, $\tilde{\varphi}$ is a $C^{\infty}$-function and is constant on an $M$-orbit in $H \times(0,+\infty) \times K$ with the $M$-action $m \cdot(h, t, k)=\left(h m^{-1}, t, m k\right)$. Since $(h, t, k) \mapsto h a_{t} k$ induces a diffeomorphism $(H \times(0,+\infty) \times K) / M \cong G-H K([29$, Theorem 9,10]), $\tilde{\varphi}$ yields a function $\varphi \in C^{\infty}((G-H K) / K ; \tau)^{H}$ such that $\varphi\left(a_{t}\right)=\phi(t)(\forall t>0)$.

Lemma 13. We have $\tau^{d, d}\left(Z_{0}^{\mathfrak{h}}\right) \xi=0\left(\forall \xi \in\left(\bigwedge^{d, d} \mathfrak{p}_{\mathbb{C}}^{*}\right)^{M}\right)$ for any $d \in \mathbb{N}$.

Proof. The operator $\tau\left(W_{0}\right)$ with $W_{0}=\sqrt{-1} \operatorname{diag}\left(1_{p},-1_{q}\right) \in \mathfrak{k}$ acts on $\mathfrak{p}_{ \pm}$by the scalar $\pm 2 \sqrt{-1}$; hence $\tau^{d, d}\left(W_{0}\right)=0$. Since the difference

$$
Z_{0}^{\mathfrak{h}}-W_{0}=\sqrt{-1} \operatorname{diag}\left(-1_{p-1}, 0,0,1_{q-1}\right)
$$

belongs to $\mathfrak{m}, \tau\left(Z_{0}^{\mathfrak{h}}-W_{0}\right)$ is zero on $\left(\bigwedge^{d, d} \mathfrak{p}_{\mathbb{C}}^{*}\right)^{M}$. From these, the conclusion follows.

4.2. Laplacians. Let $\Omega_{\mathfrak{m}}, \Omega_{\mathfrak{k}}, \Omega_{\mathfrak{h} \cap \mathfrak{k}}$ and $\Omega_{\mathfrak{g}}$ be Casimir elements of $M, K, H \cap K$ and $G$ respectively, corresponding to the invariant form $B_{\mathfrak{g}}$. Then

$$
\begin{aligned}
\Omega_{\mathfrak{k} \cap \mathfrak{h}}= & \Omega_{\mathfrak{m}}-\left(Z_{0}^{\mathfrak{h}}\right)^{2}-2 \sum_{j=1}^{q-1}\left(Z_{j}^{\mathfrak{h}} \bar{Z}_{j}^{\mathfrak{h}}+\bar{Z}_{j}^{\mathfrak{h}} Z_{j}^{\mathfrak{h}}\right), \\
\Omega_{\mathfrak{k}}= & \Omega_{\mathfrak{m}}-\left(Z_{0}^{\mathfrak{h}}\right)^{2}-2 \sum_{j=1}^{q-1}\left(Z_{j}^{\mathfrak{h}} \bar{Z}_{j}^{\mathfrak{h}}+\bar{Z}_{j}^{\mathfrak{h}} Z_{j}^{\mathfrak{h}}\right)-2 \sum_{i=1}^{p-1}\left(Z_{i}^{\mathfrak{q}} \bar{Z}_{i}^{\mathfrak{q}}+\bar{Z}_{i}^{\mathfrak{q}} Z_{i}^{\mathfrak{q}}\right), \\
\Omega_{\mathfrak{g}}= & \Omega_{\mathfrak{k}}+2 \sum_{j=0}^{q-1}\left(X_{j}^{\mathfrak{q}} \bar{X}_{j}^{\mathfrak{q}}+\bar{X}_{j}^{\mathfrak{q}} X_{j}^{\mathfrak{q}}\right)+2 \sum_{i=1}^{p-1}\left(X_{i}^{\mathfrak{h}} \bar{X}_{i}^{\mathfrak{h}}+\bar{X}_{i}^{\mathfrak{h}} X_{i}^{\mathfrak{h}}\right) \\
& +2 \sum_{i=1}^{p-1} \sum_{j=1}^{q-1}\left(X_{i j}^{\mathfrak{h}} \bar{X}_{i j}^{\mathfrak{h}}+\bar{X}_{i j}^{\mathfrak{h}} X_{i j}^{\mathfrak{h}}\right) .
\end{aligned}
$$

Let us introduce the operators

$$
\begin{aligned}
& \mathcal{S}_{\mathfrak{k}, \mathfrak{q}}=\frac{1}{2} \sum_{i=1}^{p-1} \tau\left(Z_{i}^{\mathfrak{q}} \bar{Z}_{i}^{\mathfrak{q}}+\bar{Z}_{i}^{\mathfrak{q}} Z_{i}^{\mathfrak{q}}\right)=\frac{-1}{4}\left\{\tau\left(\Omega_{\mathfrak{k}}\right)-\tau\left(\Omega_{\mathfrak{k} \cap \mathfrak{h}}\right)\right\}, \\
& \mathcal{S}_{\mathfrak{k}, \mathfrak{h}}=\frac{1}{2} \sum_{j=1}^{q-1} \tau\left(Z_{j}^{\mathfrak{h}} \bar{Z}_{j}^{\mathfrak{h}}+\bar{Z}_{j}^{\mathfrak{h}} Z_{j}^{\mathfrak{h}}\right)=\frac{-1}{4}\left\{\tau\left(\Omega_{\mathfrak{k} \cap \mathfrak{h}}\right)-\tau\left(\Omega_{\mathfrak{m}}\right)+\tau\left(Z_{0}^{\mathfrak{h}}\right)^{2}\right\}
\end{aligned}
$$

acting on $\wedge \mathfrak{p}_{\mathbb{C}}^{*}$. Let $\triangle$ be the Hodge Laplacian acting on $\mathcal{A}((G-H K) / K)$. 
Proposition 14. Let $\varphi \in C^{\infty}((G-H K) / K ; \tau)^{H}$ and set $\phi(t)=\varphi\left(a_{t}\right)(t>$ $0)$. Then $(\triangle \varphi)\left(a_{t}\right)=-\mathcal{D}_{t} \phi(t)(t>0)$ with $\mathcal{D}_{t}$ the $\left(\bigwedge \mathfrak{p}_{\mathbb{C}}^{*}\right)^{M}$-valued differential operator

$$
\begin{aligned}
\mathcal{D}_{t}= & \frac{\mathrm{d}^{2}}{\mathrm{~d} t^{2}}+((2 p-1) \tanh t+(2 q-1) \operatorname{coth} t) \frac{\mathrm{d}}{\mathrm{d} t} \\
& +\frac{4 \mathcal{S}_{\mathfrak{k}, \mathfrak{h}}}{\sinh ^{2} t}+\frac{-4 \mathcal{S}_{\mathfrak{k}, \mathfrak{q}}}{\cosh ^{2} t}+\frac{1}{4}(\operatorname{coth} t-\tanh t)^{2} \tau\left(Z_{0}^{\mathfrak{h}}\right)^{2}+\tau\left(\Omega_{\mathfrak{m}}\right) .
\end{aligned}
$$

Proof. By Kuga's lemma ([1, Chap.II, Theorem 2.5]), the action of Laplacian $\triangle$ on $\mathcal{A}_{\mathrm{c}}(G / K) \cong C_{\mathrm{c}}^{\infty}(G / K ; \tau)$ is given by the action of the Casimir $R_{\Omega_{\mathfrak{g}}}$. Hence the formula follows from (4.2), (4.1) and Lemma 1 by a direct computation.

The next lemma, which is obtained by integration-by-part, will play a key role in this paper ( $c f$. Propositions 23 and 31).

Lemma 15. Let $\alpha, \beta \in C^{\infty}\left((0,+\infty) ;\left(\bigwedge \mathfrak{p}_{\mathbb{C}}^{*}\right)^{M}\right)$ and $0<\epsilon<R$. The formula

$$
\int_{\epsilon}^{R}\left(\alpha(t) \mid \mathcal{D}_{t} \beta(t)\right) \varrho(t) \mathrm{d} t=\mathfrak{R}(\alpha, \beta ; \epsilon)-\mathfrak{R}(\alpha, \beta ; R)+\int_{\epsilon}^{R}\left(\mathcal{D}_{t} \alpha(t) \mid \beta(t)\right) \varrho(t) \mathrm{d} t
$$

holds, where

$$
\mathfrak{R}(\alpha, \beta ; t)=\varrho(t)\left\{\left(\alpha^{\prime}(t) \mid \beta(t)\right)-\left(\alpha(t) \mid \beta^{\prime}(t)\right)\right\}
$$

Proof. Fix $0<\epsilon<R$. In the following computation we use the relation $\frac{\mathrm{d}^{2}}{\mathrm{~d} t^{2}}+$ $((2 p-1) \tanh t+(2 q-1) \operatorname{coth} t) \frac{\mathrm{d}}{\mathrm{d} t}=\varrho(t)^{-1} \frac{\mathrm{d}}{\mathrm{d} t} \varrho(t) \frac{\mathrm{d}}{\mathrm{d} t}$ and the fact that the operators $\mathcal{S}_{\mathfrak{k}, \mathfrak{h}}, \mathcal{S}_{\mathfrak{k}, \mathfrak{q}}, \tau\left(Z_{0}\right)$ and $\tau\left(\Omega_{\mathfrak{m}}\right)$ are self-adjoint. Then by applying the integration-bypart twice, we have

$$
\begin{aligned}
& \int_{\epsilon}^{R}\left(\alpha(t) \mid \mathcal{D}_{t} \beta(t)\right) \varrho(t) \mathrm{d} t \\
& =\int_{\epsilon}^{R}\left(\alpha(t) \mid \frac{\mathrm{d}}{\mathrm{d} t} \varrho(t) \frac{\mathrm{d}}{\mathrm{d} t} \beta(t)\right) \mathrm{d} t \\
& \quad+\int_{\epsilon}^{R}\left(\alpha(t) \mid\left\{\frac{4 \mathcal{S}_{\mathfrak{k}, \mathfrak{h}}}{\sinh ^{2} t}+\frac{-4 \mathcal{S}_{\mathfrak{k}, \mathfrak{q}}}{\cosh ^{2} t}+\frac{(\operatorname{coth} t-\tanh t)^{2}}{4} \tau\left(Z_{0}^{\mathfrak{h}}\right)^{2}+\tau\left(\Omega_{\mathfrak{m}}\right)\right\} \beta(t)\right) \varrho(t) \mathrm{d} t \\
& =-\left(\alpha(\epsilon) \mid \varrho(\epsilon) \beta^{\prime}(\epsilon)\right)+\left(\alpha(R) \mid \varrho(R) \beta^{\prime}(R)\right)-\int_{\epsilon}^{R}\left(\frac{d}{d t} \alpha(t) \mid \varrho(t) \frac{\mathrm{d}}{\mathrm{d} t} \beta(t)\right) \mathrm{d} t \\
& \quad+\int_{\epsilon}^{R}\left(\left\{\frac{4 \mathcal{S}_{\mathfrak{k}, \mathfrak{h}}}{\sinh ^{2} t}+\frac{-4 \mathcal{S}_{\mathfrak{k}, \mathfrak{q}}}{\cosh ^{2} t}+\frac{(\operatorname{coth} t-\tanh t)^{2}}{4} \tau\left(Z_{0}^{\mathfrak{h}}\right)^{2}+\tau\left(\Omega_{\mathfrak{m}}\right)\right\} \alpha(t) \mid \beta(t)\right) \varrho(t) \mathrm{d} t \\
& =-\left(\alpha(\epsilon) \mid \varrho(\epsilon) \beta^{\prime}(\epsilon)\right)+\left(\alpha(R) \mid \varrho(R) \beta^{\prime}(R)\right)-\left(\varrho(R) \alpha^{\prime}(R) \mid \beta(R)\right)+\left(\varrho(\epsilon) \alpha^{\prime}(\epsilon) \mid \beta(\epsilon)\right)
\end{aligned}
$$




$$
\begin{aligned}
& \quad+\int_{\epsilon}^{R}\left(\frac{\mathrm{d}}{\mathrm{d} t} \varrho(t) \frac{\mathrm{d}}{\mathrm{d} t} \alpha(t) \mid \beta(t)\right) \mathrm{d} t \\
& \quad+\int_{\epsilon}^{R}\left(\left\{\frac{4 \mathcal{S}_{\mathfrak{B}, \mathfrak{h}}}{\sinh ^{2} t}+\frac{-4 \mathcal{S}_{\mathfrak{e}, \mathfrak{q}}}{\cosh ^{2} t}+\frac{(\operatorname{coth} t-\tanh t)^{2}}{4} \tau\left(Z_{0}^{\mathfrak{h}}\right)^{2}+\tau\left(\Omega_{\mathfrak{m}}\right)\right\} \alpha(t) \mid \beta(t)\right) \varrho(t) \mathrm{d} t \\
& =\mathfrak{R}(\alpha, \beta ; \epsilon)-\mathfrak{R}(\alpha, \beta ; R)+\int_{\epsilon}^{R}\left(\mathcal{D}_{t} \alpha(t) \mid \beta(t)\right) \varrho(t) \mathrm{d} t .
\end{aligned}
$$

4.3. $\partial \bar{\partial}$-operator. Since $(G-H K) / K$ is an open subset of the complex manifold $G / K$, we have the usual operators $\partial, \bar{\partial}$ and their formal adjoints $\partial^{*}, \bar{\partial}^{*}$ acting on $\mathcal{A}((G-H K) / K) \cong C^{\infty}((G-H K) / K ; \tau)$ :

$$
\begin{aligned}
\partial & =\sum_{i=1}^{p} \sum_{j=1}^{q} e\left(\omega_{i j}\right) R_{E_{i, p+j}}, & \bar{\partial} & =\sum_{i=1}^{p} \sum_{j=1}^{q} e\left(\bar{\omega}_{i j}\right) R_{E_{p+j, i}}, \\
\partial^{*} & =-\sum_{i=1}^{p} \sum_{j=1}^{q} e^{*}\left(\omega_{i j}\right) R_{E_{p+j, i}}, & \bar{\partial}^{*} & =-\sum_{i=1}^{p} \sum_{j=1}^{q} e^{*}\left(\bar{\omega}_{i j}\right) R_{E_{i, p+j}} .
\end{aligned}
$$

In order to describe the composite operator $\partial \bar{\partial}$ and $\bar{\partial}^{*} \partial^{*}$ on the $H$-invariant forms concretely, let us introduce operators acting on $\Lambda \mathfrak{p}_{\mathbb{C}}^{*}$ :

$$
\begin{aligned}
\mathcal{P}_{+} & =\sum_{i=1}^{p-1} e\left(\bar{\omega}_{i 1}\right) \tau\left(\bar{Z}_{i}^{\mathfrak{q}}\right), \quad \mathcal{P}_{-}=\sum_{i=1}^{p-1} e\left(\omega_{i 1}\right) \tau\left(Z_{i}^{\mathfrak{q}}\right), \quad e\left(\eta_{\mathfrak{h}}\right)=\sum_{i=1}^{p-1} e\left(\omega_{i 1} \wedge \bar{\omega}_{i 1}\right), \\
\mathcal{R}_{+} & =\sum_{j=1}^{q-1} e\left(\bar{\omega}_{p, j+1}\right) \tau\left(\bar{Z}_{j}^{\mathfrak{h}}\right), \quad \mathcal{R}_{-}=\sum_{j=1}^{q-1} e\left(\omega_{p, j+1}\right) \tau\left(Z_{j}^{\mathfrak{h}}\right), \\
e\left(\eta_{\mathfrak{q}}\right) & =\sum_{j=1}^{q-1} e\left(\omega_{p, j+1} \wedge \bar{\omega}_{p, j+1}\right),
\end{aligned}
$$

and

$$
\begin{aligned}
\mathcal{A} & =e\left(\eta_{\mathfrak{h}}\right)+\frac{1}{2} e\left(\omega_{0} \wedge \bar{\omega}_{0}\right)-e\left(\bar{\omega}_{0}\right) \mathcal{P}_{-}+e\left(\omega_{0}\right) \mathcal{P}_{+} \\
\mathcal{B} & =e\left(\eta_{\mathfrak{q}}\right)+\frac{1}{2} e\left(\omega_{0} \wedge \bar{\omega}_{0}\right)-e\left(\bar{\omega}_{0}\right) \mathcal{R}_{-}+e\left(\omega_{0}\right) \mathcal{R}_{+} \\
\mathcal{C} & =e\left(\omega_{0}\right)\left(\mathcal{P}_{+}+\mathcal{R}_{+}\right)+\mathcal{P}_{-} \mathcal{R}_{+}+\mathcal{R}_{-} \mathcal{P}_{+}
\end{aligned}
$$

Here we set $\omega_{0}=\omega_{p, 1}$. 
Proposition 16. Let $\varphi \in C^{\infty}((G-H K) / K ; \tau)^{H}$ and set $\phi(t)=\varphi\left(a_{t}\right)(t>0)$. Then $(\partial \bar{\partial} \varphi)\left(a_{t}\right)=\mathcal{E}_{t} \phi(t)(t>0)$ with $\mathcal{E}_{t}$ the $\left(\bigwedge \mathfrak{p}_{\mathbb{C}}^{*}\right)^{M}$-valued differential operator

$$
\begin{aligned}
\mathcal{E}_{t} & =\frac{1}{4} e\left(\omega_{0} \wedge \bar{\omega}_{0}\right) \frac{\mathrm{d}^{2}}{\mathrm{~d} t^{2}}+\frac{1}{2}(\tanh t \mathcal{A}+\operatorname{coth} t \mathcal{B}) \frac{\mathrm{d}}{\mathrm{d} t} \\
& +\tanh ^{2} t \mathcal{P}_{-} \mathcal{P}_{+}+\operatorname{coth}^{2} t \mathcal{R}_{-} \mathcal{R}_{+}+\mathcal{C} \\
& +\frac{\sqrt{-1}}{4}\left(1+\tanh ^{2} t\right)\left(\frac{2}{\sqrt{-1}} e\left(\eta_{\mathfrak{h}}\right)-e\left(\bar{\omega}_{0}\right) \mathcal{P}_{-}-e\left(\omega_{0}\right) \mathcal{P}_{+}\right) \tau\left(Z_{0}^{\mathfrak{h}}\right) \\
& +\frac{\sqrt{-1}}{4}\left(1+\operatorname{coth}^{2} t\right)\left(\frac{2}{\sqrt{-1}} e\left(\eta_{\mathfrak{q}}\right)-e\left(\bar{\omega}_{0}\right) \mathcal{R}_{-}-e\left(\omega_{0}\right) \mathcal{R}_{+}\right) \tau\left(Z_{0}^{\mathfrak{h}}\right) \\
& +\frac{\sqrt{-1}}{2} e\left(\omega_{0} \wedge \bar{\omega}_{0}\right) \tau\left(Z_{0}^{\mathfrak{h}}\right)+\frac{1}{16}(\tanh t+\operatorname{coth} t)^{2} \tau\left(Z_{0}^{\mathfrak{h}}\right)^{2} .
\end{aligned}
$$

We have $\left(\bar{\partial}^{*} \partial^{*} \varphi\right)\left(a_{t}\right)=\varepsilon_{t}^{*} \phi(t)(\forall t>0)$ with $\varepsilon_{t}^{*}$ the formal adjoint of $\varepsilon_{t}$ defined by

$$
\begin{aligned}
& \int_{0}^{+\infty}\left(\mathcal{E}_{t} \alpha(t) \mid \beta(t)\right) \varrho(t) \mathrm{d} t=\int_{0}^{+\infty}\left(\alpha(t) \mid \mathcal{E}_{t}^{*} \beta(t)\right) \varrho(t) \mathrm{d} t \\
&\left(\forall \alpha, \beta \in C_{\mathrm{c}}^{\infty}\left((0,+\infty) ;\left(\bigwedge \mathfrak{p}_{\mathbb{C}}^{*}\right)^{M}\right) .\right.
\end{aligned}
$$

Proof. Using the expression of $\partial$ and $\bar{\partial}$ above and also the formulas in Lemma 1 , we prove the formula $(\partial \bar{\partial} \varphi)\left(a_{t}\right)=\mathcal{E}_{t} \phi(t)$ by a direct computation.

Lemma 17. Let $\left.\alpha, \beta \in C^{\infty}((0,+\infty)) ;\left(\bigwedge \mathfrak{p}_{\mathbb{C}}^{*}\right)^{M}\right)$ and $0<\epsilon<R$. The formula

$$
\int_{\epsilon}^{R}\left(\mathcal{E}_{t} \beta(t) \mid \alpha(t)\right) \varrho(t) \mathrm{d} t=\mathfrak{S}(R)-\mathfrak{S}(\epsilon)+\int_{\epsilon}^{R}\left(\beta(t) \mid \mathcal{E}_{t}^{*} \alpha(t)\right) \varrho(t) \mathrm{d} t
$$

holds, where

$$
\begin{aligned}
\mathfrak{S}(t)= & \frac{\varrho(t)}{4}\left\{\left(e\left(\omega_{0} \wedge \bar{\omega}_{0}\right) \beta^{\prime}(t) \mid \alpha(t)\right)-\left(e\left(\omega_{0} \wedge \bar{\omega}_{0}\right) \beta(t) \mid \alpha^{\prime}(t)\right)\right. \\
& +\tanh t\left(\left(2 \mathcal{A}-(2 p-1) e\left(\omega_{0} \wedge \bar{\omega}_{0}\right)\right) \beta(t) \mid \alpha(t)\right) \\
& \left.+\operatorname{coth} t\left(\left(2 \mathcal{B}-(2 q-1) e\left(\omega_{0} \wedge \bar{\omega}_{0}\right)\right) \beta(t) \mid \alpha(t)\right)\right\} .
\end{aligned}
$$

Proof. Similar to the proof of Lemma 15.

\section{THE SECONDARY SPHERICAL FUNCTIONS}

Set $\rho_{0}=p+q-1$. In this section, we fix an integer $0 \leqslant d \leqslant q$ and set

$$
D^{(d)}=\mathbb{C}-\left\{\rho_{0}-2(q-d+n) \mid n \in \mathbb{N}\right\} .
$$

Here is the main theorem of this section. 
Theorem 18. (1) There exists a unique family $\varphi_{s}^{(d)}\left(s \in D^{(d)}\right)$ of functions with the properties:

(i) For $s \in D^{(d)}, \varphi_{s}^{(d)} \in C^{\infty}\left((G-H K) / K ; \tau^{d, d}\right)^{H}$.

(ii) For each $g \in G-H K$, the value $\varphi_{s}^{(d)}(g)$ depends on $s \in D^{(d)}$ holomorphically.

(iii) For each $s \in D^{(d)}$,

$$
\Omega_{\mathfrak{g}} \varphi_{s}^{(d)}(g)=\left(s^{2}-\rho_{0}^{2}\right) \varphi_{s}^{(d)}(g), \quad(g \in G-H K) .
$$

(iv) It has the 'small-time behavior'

$$
\lim _{t \rightarrow+0} t^{2(q-1)} \varphi_{s}^{(d)}\left(a_{t}\right)=\Lambda^{q-d}\left(* \operatorname{vol}_{\mathfrak{p} \cap \mathfrak{h}}\right) .
$$

(v) It has the 'large-time behavior'

$$
\varphi_{s}^{(d)}\left(a_{t}\right)=O\left(e^{-\left(\operatorname{Re}(s)+\rho_{0}\right) t}\right), \quad(t \rightarrow+\infty) .
$$

(2) The radial value $\varphi_{s}^{(d)}\left(a_{t}\right)$ is given by the explicit formula

$$
\varphi_{s}^{(d)}\left(a_{t}\right)=\sum_{\kappa=0}^{d} F_{\kappa}(s ; t) \theta_{\kappa}^{(d)}, \quad(t>0) .
$$

Here for each $\kappa \in \mathbb{N}, s \in \mathbb{C}$ and $t>0$, we set

$$
\begin{aligned}
F_{\kappa}(s ; t) & =\frac{\Gamma\left(\frac{s+\rho_{0}}{2}+\kappa\right) \Gamma\left(\frac{s-\rho_{0}}{2}+q-\kappa\right)}{\Gamma(s+1) \Gamma(q-1)} \\
& \times(\cosh t)^{-\left(s+\rho_{0}\right)}{ }_{2} F_{1}\left(\frac{s+\rho_{0}}{2}+\kappa, \frac{s-\rho_{0}}{2}+q-\kappa ; s+1 ; \frac{1}{\cosh ^{2} t}\right) .
\end{aligned}
$$

The next corollary says that only the function $\varphi_{s}^{(q)}$ is essential, from which others $\varphi_{s}^{(d)}$ with smaller bidegree $(d, d)$ are obtained by successive application of $\Lambda$.

Corollary 19. We have $\Lambda \varphi_{s}^{(d)}=\varphi_{s}^{(d-1)}$ whenever $1 \leqslant d \leqslant q, s \in D^{(d)}$.

Proof. This follows immediately from the explicit formula (5.1) and the last statement of Proposition 11 (2). Another proof is first to check that $\Lambda \varphi_{s}^{(d)}$ has the same properties (i) to (v) as $\varphi_{s}^{(d-1)}$, which is easy, and then to use the uniqueness of $\varphi_{s}^{(d-1)}$. 
5.1. Some properties of the secondary spherical functions. In this subsection, we fix a family of functions $\varphi_{s}^{(d)}\left(s \in D^{(d)}\right)$ satisfying the conditions (i), (ii), (iii), (iv) and (v) in Theorem 18. Starting with these five properties, we deduce several substantial results which will be used not only to prove Theorem 18 but also to study Poincaré series in the next section .

First of all, to study the local behavior of $\varphi_{s}^{(d)}\left(a_{t}\right)$ near the boundary points $t=0,+\infty$, we introduce the local coordinate $z=\tanh ^{2} t$ around $t=0$ and the one $\zeta=\frac{1}{\cosh ^{2} t}$ around $t=+\infty$.

Proposition 20. (1) There exist $0<\epsilon<1$ and a $\left(\bigwedge^{d, d} \mathfrak{p}_{\mathbb{C}}^{*}\right)^{M}$-valued holomorphic function $\mathrm{R}(s, \zeta)$ on $D^{(d)} \times\{|\zeta|<\epsilon\}$ such that

$$
\varphi_{s}^{(d)}\left(a_{t}\right)=\zeta^{\left(s+\rho_{0}\right) / 2} \mathrm{R}(s, \zeta), \quad\left(s \in D^{(d)}, \zeta \in(0, \epsilon)\right) .
$$

(2) There exist $N \in \mathbb{N}, 0<\delta<1$ and $\left(\bigwedge^{d, d} \mathfrak{p}_{\mathbb{C}}^{*}\right)^{M}$-valued holomorphic functions $\mathrm{P}_{h}(s, z)(0 \leqslant h \leqslant N)$ on $D^{(d)} \times\{|z|<\delta\}$ such that $\mathrm{P}_{0}(s, 0)=$ $\Lambda^{q-d}\left(* \operatorname{vol}_{\mathfrak{p} \cap \mathfrak{h}}\right)$ and

$$
\varphi_{s}^{(d)}\left(a_{t}\right)=z^{-(q-1)}\left\{\mathrm{P}_{0}(s, z)+\sum_{h=1}^{N} z(\log z)^{h} \mathrm{P}_{h}(s, z)\right\}, \quad\left(s \in D^{(d)}, z \in(0, \delta)\right) .
$$

Proof. Set $V=\left(\bigwedge \mathfrak{p}_{\mathbb{C}}^{*}\right)^{M}$. From the condition (iii), $\phi(t)=\varphi_{s}^{(d)}\left(a_{t}\right)$ satisfies the differential equation $\mathcal{D}_{t} \phi(t)=\left(s^{2}-\rho_{0}^{2}\right) \phi(t)$ on $t>0$. Here $\mathcal{D}_{t}$ is the differential operator given in Proposition 14. By the change of variable $z=\tanh ^{2} t$, which yields a diffeomorphism from $t>0$ to $0<z<1$, the equation $\mathcal{D}_{t} \phi(t)=\left(s^{2}-\right.$ $\left.\rho_{0}^{2}\right) \phi(t)$ becomes

$$
\left\{\frac{\mathrm{d}^{2}}{\mathrm{~d} z^{2}}+\left(\frac{q}{z}+\frac{\rho_{0}-1}{1-z}\right) \frac{\mathrm{d}}{\mathrm{d} z}+\mathrm{Q}(s ; z)\right\} \phi(z)=0, \quad(0<z<1)
$$

with

$$
\mathrm{Q}(s ; z)=\frac{-\mathcal{S}_{\mathfrak{L}, \mathfrak{q}}}{z(1-z)}+\frac{\mathcal{S}_{\mathfrak{k}, \mathfrak{b}}}{z^{2}(1-z)}+\frac{s^{2}-\rho_{0}^{2}}{4 z(1-z)^{2}} 1_{V}(\in \operatorname{End}(V)) .
$$

This implies that the function $\Phi(z)=\left[\begin{array}{c}\phi(z) \\ z \phi^{\prime}(z)\end{array}\right]\left(\in V^{2 \oplus}\right)$ is a solution of the first order differential equation

$$
\frac{d \Phi}{d z}(z)=\mathrm{A}(s ; z) \Phi(z), \quad \mathrm{A}(s ; z)=\left[\begin{array}{cc}
0 & \frac{1}{z} 1_{V} \\
-z \mathrm{Q}(s ; z) & \left(\frac{1-q}{z}+\frac{1-\rho_{0}}{1-z}\right) 1_{V}
\end{array}\right] .
$$


Since $z=0$ is a simple pole of $\mathrm{A}(s ; z), z=0$ is a regular singular point of the equation (5.5) ([3, Theorem 2.(p.111)]). The integer $-(q-1)$ is one of its characteristic roots at $z=0$, which are the eigenvalues of the operator

$$
\operatorname{Res}_{z=0} \mathrm{~A}(s ; z)=\mathrm{A}_{0}=\left[\begin{array}{cc}
0 & 1_{V} \\
-\mathcal{S}_{\mathfrak{k}, \mathfrak{h}} & (1-q) 1_{V}
\end{array}\right] .
$$

By Lemma 21 below, the characteristic roots are integers; hence by [3, Theorem $4.2(\mathrm{p} .121)]$ the solution $\Phi(z)$ has to be of the form $\Phi(z)=z^{\lambda_{0}} \sum_{h=0}^{N}(\log z)^{h} \tilde{\mathrm{F}}_{h}(s, z)$ with $\lambda_{0}$ the smallest characteristic root. Here $\tilde{\mathrm{F}}_{h}(s, z)$ 's are $V^{2 \oplus_{\text {-valued holomor- }}}$ phic functions on $D^{(s)} \times\{|z|<\delta\}$ with small $\delta>0$. Let $\tilde{P}_{h}(s, z)$ be the first projection of $\tilde{\mathrm{F}}_{h}(s, z)$ to $V$. Then we obtain this local expression of $\phi(z)$ :

$$
\phi(z)=z^{\lambda_{0}} \sum_{h=0}^{N}(\log z)^{h} \tilde{\mathrm{P}}_{h}(s, z), \quad(0<z<\delta) .
$$

By the condition (iv), the function $z^{q-1} \phi(z)$ has the limit $\Lambda^{q-d}\left(* \operatorname{vol}_{\mathfrak{p} \cap \mathfrak{h}}\right)$ as $z \rightarrow+0$ with $z \in \mathbb{R}$. This implies that $\mathrm{P}_{0}(s, z)=z^{\lambda_{0}+q-1} \tilde{\mathrm{P}}_{0}(s, z)$ should be holomorphic at $z=0$ with constant term $\Lambda^{q-d}\left(* \operatorname{vol}_{\mathfrak{p} \cap \mathfrak{h}}\right)$ and that $z^{\lambda_{0}+q-1} \tilde{\mathrm{P}}_{h}(s, z)$ should be of the form $z \mathrm{P}_{h}(s, z)$ with $\mathrm{P}_{h}(s, z)$ holomorphic at $z=0$. This completes the proof of (5.3). The proof of (5.2) is similar.

Lemma 21. The eigenvalues of the linear operator $\mathrm{A}_{0}$ coincides with the set of numbers $\lambda \in \mathbb{C}$ such that $\operatorname{det}\left(\mathcal{S}_{\mathfrak{k}, \mathfrak{h}}+\lambda(\lambda+q-1)\right)=0$, which consists of integers.

Proof. For a given $w=\left(v_{1}, v_{2}\right) \in V^{2}$, the equation $\mathrm{A}_{0} w=\lambda w$ is equivalent to the system of equations $v_{2}=\lambda v_{1},\left\{\mathcal{S}_{\mathfrak{k}, \mathfrak{h}}+\lambda(\lambda+q-1)\right\} v_{1}=0$. This shows the first assertion of our lemma.

Let $\Lambda \mathfrak{p}_{\mathbb{C}}^{*}=\bigoplus_{i \in I} W_{i}$ be a $K \cap H$-irreducible decomposition. Taking $M$-fixed part, we have the decomposition $V=\bigoplus_{i \in I} W_{i}^{M}$, by which the Casimir operator $\Omega_{\mathfrak{k} \cap \mathfrak{h}}$ is diagonalized. Since $\mathcal{S}_{\mathfrak{k}, \mathfrak{h}}=\frac{-1}{4} \tau\left(\Omega_{\mathfrak{k} \cap \mathfrak{h}}\right)$ on $V$ by (4.4), the eigenvalues of $\mathcal{S}_{\mathfrak{k}, \mathfrak{h}}$ on $V$ are computable from this decomposition. Identify $\mathfrak{k}_{\mathbb{C}} \cap \mathfrak{h}_{\mathbb{C}}=\mathfrak{g l}_{p-1}(\mathbb{C}) \oplus \mathbb{C} \oplus$ $\mathfrak{g l}_{q}(\mathbb{C})$ and $\mathfrak{m}_{\mathbb{C}}=\mathfrak{g l}_{p-1}(\mathbb{C}) \oplus \mathbb{C} \oplus \mathfrak{g l}_{q-1}(\mathbb{C})$ naturally; then by $\mathfrak{g l}_{q-1} \rightarrow \mathfrak{g l}_{q}$-branching rule $\left(\left[6\right.\right.$, Theorem 8.1 .1 (p.350)]), the highest weight of $W_{i}$ such that $W_{i}^{M} \neq\{0\}$ is of the form $[a, 0, \cdots, 0,-a] \oplus[0] \oplus[0, \ldots, 0]$ with $a \in \mathbb{N}$. The eigenvalue of $\Omega_{\mathfrak{k} \cap \mathfrak{h}}$ on this $W_{i}$ is easily calculated as $4 a(a+q-1)$. Hence $\mathcal{S}_{\mathfrak{k}, \mathfrak{h}} \mid W_{i}^{M}=-a(a+p-1)$. The argument so far shows an eigenvalue of $\mathcal{S}_{\mathfrak{k}, \mathfrak{h}}$ on $V$ belongs to $\{-a(a+q-1) \mid a \in \mathbb{N}\}$.

If $\lambda$ satisfies $\operatorname{det}\left(\mathcal{S}_{\mathfrak{k}, \mathfrak{h}}+\lambda(\lambda+q-1)\right)=0$, then $\lambda(\lambda+q-1)=a(a+q-1)$ with some $a \in \mathbb{N}$. Hence $\lambda=a, 1-q-a$, in particular $\lambda \in \mathbb{Z}$. 
Since $H K$ is a zero set of $G$ with respect to the Haar measure, the form $\varphi_{s}^{(d)}$ is regarded as a measurable form on $G / K$.

Lemma 22. The measurable form $\varphi_{s}^{(d)}$ on $G / K$ is locally integrable.

Proof. Let $\left\{U_{n}\right\}_{n \in \mathbb{N}}$ be an open covering of $H$ by relatively compact subsets. The sets $G_{\epsilon, n}=U_{n} \exp \left([0, \epsilon] Y_{0}\right) K,(\epsilon>0, n \in \mathbb{N})$ form an open covering of $G$ by relatively compact sets. Fix $\epsilon>0$ and $n \in \mathbb{N}$. From the property (iv), there exists a constant $C_{\epsilon}>0$ such that $\left\|\varphi_{s}^{(d)}\left(a_{t}\right)\right\| \leqslant C_{\epsilon} t^{-2(q-1)}(\forall t \in[0, \epsilon])$. Using this estimation, by Lemma 2 , we have

$$
\int_{G_{\epsilon, n}}\left\|\varphi_{s}^{(d)}(g)\right\| \mathrm{d} g=\operatorname{vol}\left(U_{n}\right) \int_{0}^{\epsilon}\left\|\varphi^{(d)}\left(a_{t}\right)\right\| \varrho(t) \mathrm{d} t \leqslant C_{\epsilon} \operatorname{vol}\left(U_{n}\right) \int_{0}^{\epsilon} t^{-2(q-1)} \varrho(t) \mathrm{d} t .
$$

Since $t^{-2(q-1)} \varrho(t)=O(t),(|t| \leqslant \epsilon)$ by $(2.4)$, the last integral is convergent.

Proposition 23. Assume $\operatorname{Re}(s)>\rho_{0}$. Then the $(d, d)$-current $\varphi_{s}^{(d)}$ on $G / K$ satisfies the differential equation:

$$
\left(\triangle+s^{2}-\rho_{0}^{2}\right) \varphi_{s}^{(d)}=\frac{4 \pi^{q}}{\Gamma(q-1)} \Lambda^{q-d} \delta_{H / H \cap K} .
$$

Proof. Let $f \in \mathcal{A}_{\mathrm{c}}(G / K)$. Then

$$
\begin{aligned}
\left\langle\left(\triangle+s^{2}-\rho_{0}^{2}\right) \varphi_{s}^{(d)}, * \bar{f}\right\rangle & =\left\langle\varphi_{s}^{(d)},\left(\triangle+s^{2}-\rho_{0}^{2}\right) * \bar{f}\right\rangle \\
& =\int_{0}^{+\infty}\left(\varphi_{s}^{(d)}\left(a_{t}\right) \mid \int_{H}\left(\triangle+\bar{s}^{2}-\rho_{0}^{2}\right) f\left(h a_{t}\right) d h\right) \varrho(t) \mathrm{d} t .
\end{aligned}
$$

Since $f$ is of compact support, the integral $f^{H}(g)=\int_{H} f(h g) \mathrm{d} h(g \in G)$ converges absolutely and defines an $H$-invariant function $f^{H} \in C^{\infty}(G / K ; \tau)^{H}$. Moreover, $f \mapsto f^{H}$ is a $Z\left(\mathfrak{g}_{\mathbb{C}}\right)$-homomorphism from $\mathcal{A}_{\mathrm{c}}(G / K)$ to $C^{\infty}(G / K ; \tau)^{H}$. Here $Z\left(\mathfrak{g}_{\mathbb{C}}\right)$ is the center of the universal enveloping algebra of $\mathfrak{g}_{\mathbb{C}}$. By this remark and Proposition 14 , we have

$$
\begin{aligned}
\int_{H}\left(\triangle+\bar{s}^{2}-\rho_{0}^{2}\right) f(h g) \mathrm{d} h & =\left(\triangle+\bar{s}^{2}-\rho_{0}^{2}\right) f^{H}\left(a_{t}\right) \\
& =\left(-\mathcal{D}_{t}+\bar{s}^{2}-\rho_{0}^{2}\right) f^{H}\left(a_{t}\right)
\end{aligned}
$$

for $t>0$. Use this formula to obtain the expression of the paring (5.7) in terms of $f^{H}$

$$
\left\langle\left(\triangle+s^{2}-\rho_{0}^{2}\right) \varphi_{s}^{(d)}, * \bar{f}\right\rangle=\int_{0}^{+\infty}\left(\varphi_{s}^{(d)}\left(a_{t}\right) \mid\left(-\mathcal{D}_{t}+\bar{s}^{2}-\rho_{0}^{2}\right) f^{H}\left(a_{t}\right)\right) \varrho(t) \mathrm{d} t .
$$


The Secondary Spherical Functions and Automorphic Green ... 1003

Fix $0<\epsilon<R$ and apply Lemma 15 with $\alpha(t)=\varphi_{s}^{(d)}\left(a_{t}\right)$ and $\beta(t)=f^{H}\left(a_{t}\right)$. Then

$$
\int_{\epsilon}^{R}\left(\varphi_{s}^{(d)}\left(a_{t}\right) \mid\left(-\mathcal{D}_{t}+\bar{s}^{2}-\rho_{0}^{2}\right) f^{H}\left(a_{t}\right)\right) \varrho(t) \mathrm{d} t=\mathfrak{R}(R)-\mathfrak{R}(\epsilon)
$$

since $\left(-\mathcal{D}_{t}+s^{2}-\rho_{0}^{2}\right) \varphi_{s}^{(d)}\left(a_{t}\right)=0(\forall t>0)$ by the property (i) and Proposition 14 . Here

$$
\mathfrak{R}(t)=-\varrho(t)\left(\varphi_{s}^{(d)}\left(a_{t}\right) \mid \frac{d}{d t} f^{H}\left(a_{t}\right)\right)+\varrho(t)\left(\frac{\mathrm{d}}{\mathrm{d} t} \varphi_{s}^{(d)}\left(a_{t}\right) \mid f^{H}\left(a_{t}\right)\right) .
$$

Let us compute the limit of $\mathfrak{R}(\epsilon)$ as $\epsilon \rightarrow+0$. By differentiation of (5.3), we have

$$
\begin{aligned}
\frac{\mathrm{d}}{\mathrm{d} t} \varphi_{s}^{(d)}\left(a_{t}\right)=- & (q-1) z^{-q} \frac{\mathrm{d} z}{\mathrm{~d} t}\left\{\mathrm{P}_{0}(s, z)+\sum_{h=1}^{N} z(\log z)^{h} \mathrm{P}_{h}(s, z)\right\} \\
& +z^{-q+1} \frac{\mathrm{d} z}{\mathrm{~d} t}\left\{\frac{\partial \mathrm{P}_{0}}{\partial z}(s, z)+\sum_{h=1}^{N}(\log z+h)(\log z)^{h-1} \mathrm{P}_{h}(s, z)\right. \\
& \left.+\sum_{h=1}^{N} z(\log z)^{h} \frac{\partial \mathrm{P}_{h}}{\partial z}(s, z)\right\}
\end{aligned}
$$

for small $z$. Since $\mathrm{P}_{0}(s, z)=\Lambda^{q-d}\left(* \operatorname{vol}_{\mathfrak{p} \cap \mathfrak{h}}\right)+O(z), \mathrm{P}_{h}(s, z)=O(1), \frac{\partial \mathrm{P}_{h}}{\partial z}(s, z)=$ $O(1)$ and $\frac{\mathrm{d} z}{\mathrm{~d} t}=2 z^{\frac{1}{2}}(1-z)$, we have the estimation

$$
\begin{aligned}
\frac{\mathrm{d}}{\mathrm{d} t} \varphi_{s}^{(d)}\left(a_{t}\right)= & -2(q-1) z^{\frac{1}{2}-q}(1+O(z))\left\{\Lambda^{q-d}\left(* \operatorname{vol}_{\mathfrak{p} \cap \mathfrak{h}}\right)+O(z)+\sum_{h=1}^{N} O\left(z(\log z)^{h}\right)\right\} \\
& +z^{1-q}\left\{O(1)+\sum_{h=1}^{N} O\left((\log z)^{h}\right)+\sum_{h=1}^{N} O\left(z^{\frac{3}{2}}(\log z)^{h}\right)\right\} \\
= & -2(q-1) z^{\frac{1}{2}-q} \Lambda^{q-d}\left(* \operatorname{vol}_{\mathfrak{p} \cap \mathfrak{h}}\right)+O\left(z^{1-q}(\log z)^{N}\right) \\
= & -2(q-1) t^{-2 q+1} \Lambda^{q-d}\left(* \operatorname{vol}_{\mathfrak{p} \cap \mathfrak{h}}\right)+O\left(t^{2-2 q}(\log t)^{N}\right)
\end{aligned}
$$

for small $t>0$. Since $\frac{\mathrm{d}}{\mathrm{d} t} f^{H}\left(a_{t}\right)$ is continuous at $t=0$ and $\varphi_{s}^{(d)}\left(a_{t}\right)=O\left(t^{-2 q+2}\right)$, $\varrho(t)=\frac{2 \pi^{q}}{\Gamma(q)} t^{2 q-1}(1+O(t))$ for small $t$, the first term of (5.10) is majorized by $O\left(\varrho(t) t^{-2 q+2}\right)=O(t)$. Hence

$$
\begin{aligned}
\lim _{\epsilon \rightarrow+0} \mathfrak{R}(\epsilon) & =\lim _{\epsilon \rightarrow+0} \varrho(t)\left(\frac{\mathrm{d}}{\mathrm{d} t} \varphi_{s}^{(d)}\left(a_{t}\right) \mid f^{H}\left(a_{t}\right)\right) \\
& =\frac{-4 \pi^{q}}{\Gamma(q-1)}\left(\Lambda^{q-d}\left(* \operatorname{vol}_{\mathfrak{p} \cap \mathfrak{h}}\right) \mid f^{H}(e)\right) .
\end{aligned}
$$

Let us compute the limit of $\Re(R)$ as $R \rightarrow+\infty$. Since $f$ is of compact support in $G$ and $H \exp \left([0, R) Y_{0}\right) K,(R>0)$ is an open covering of $G$, there exists an 
$R_{0}>0$ such that $\operatorname{supp}(f) \subset H \exp \left(\left[0, R_{0}\right] Y_{0}\right) K$. Hence $f^{H}\left(a_{t}\right)=0\left(\forall t>R_{0}\right)$. This yields $\mathfrak{R}(R)=0\left(\forall R>R_{0}\right)$, especially

$$
\lim _{R \rightarrow+\infty} \mathfrak{R}(R)=0 .
$$

By (5.8), (5.9), (5.11), (5.12) and Lemma 3, we obtain

$$
\begin{aligned}
\left\langle\left(\triangle+s^{2}-\rho_{0}^{2}\right) \varphi_{s}^{(d)}, * \bar{f}\right\rangle & =\frac{4 \pi^{q}}{\Gamma(q-1)} \int_{H}\left(\Lambda^{q-d}\left(* \operatorname{vol}_{\mathfrak{p} \cap \mathfrak{h}}\right) \mid f(h)\right) \mathrm{d} h \\
& =\frac{4 \pi^{q}}{\Gamma(q-1)}\left\langle\Lambda^{q-d} \delta_{H / H \cap K}, * \bar{f}\right\rangle .
\end{aligned}
$$

\subsection{The proof of Theorem 18.}

5.2.1. The construction of a solution. By Proposition 14 and Lemma 12, finding a function $\varphi_{s}^{(d)}$ with the properties (i) and (iii) is equivalent to finding the function $\phi(t)=\varphi_{s}^{(d)}\left(a_{t}\right)$ on $t>0$ which takes its values in the vector space $\left(\bigwedge \mathfrak{p}_{\mathbb{C}}^{*}\right)^{M}$ and satisfies the ordinary differential equation

$$
\mathcal{D}_{t} \phi(t)=\left(s^{2}-\rho_{0}^{2}\right) \phi(t) .
$$

We search for a solution of (5.13) assuming the form

$$
\phi(t)=\sum_{\kappa=0}^{d} \phi_{\kappa}(t) \theta_{\kappa}^{(d)} \quad(t>0)
$$

with $d+1$ unknown functions $\phi_{\kappa}(t)$. By (3.10), the condition (iv) for (5.14) is equivalent to requiring

$$
\lim _{t \rightarrow+0} t^{2(q-1)} \phi_{\kappa}(t)=1 .
$$

For the function (5.14) to meet the condition (v), we also have to require

$$
\phi_{\kappa}(t)=O\left(e^{-\left(\operatorname{Re}(s)+\rho_{0}\right) t}\right), \quad(t \rightarrow+\infty) .
$$

By Lemma 13 and $H \cap K$-invariance of $\theta_{\kappa}^{(d)}$ (Lemma 5$)$, the operators $\tau\left(Z_{0}^{\mathfrak{h}}\right), \mathcal{S}_{\mathfrak{k}, \mathfrak{h}}$, $\tau\left(\Omega_{\mathfrak{k} \cap \mathfrak{h}}\right)$ and $\tau\left(\Omega_{\mathfrak{m}}\right)$ are all zero when applied to $\theta_{\kappa}^{(d)}$. By (4.3) and Proposition 11 (1), we have $\mathcal{S}_{\mathfrak{k}, \mathfrak{q}} \theta_{\kappa}^{(d)}=\frac{-1}{4} \tau\left(\Omega_{\mathfrak{k}}\right) \theta_{\kappa}^{(d)}=-\kappa(\kappa+p-1) \theta_{\kappa}^{(d)}$. Hence the equation (5.13) is simplified as

$$
\left\{\frac{\mathrm{d}^{2}}{\mathrm{~d} t^{2}}+((2 p-1) \tanh t+(2 q-1) \operatorname{coth} t) \frac{\mathrm{d}}{\mathrm{d} t}+\frac{4 \kappa(\kappa+p-1)}{\cosh ^{2} t}+\rho_{0}^{2}-s^{2}\right\} \phi_{\kappa}(t)=0 .
$$


Lemma 24. Let $p, q, A, B, C \in \mathbb{C}$ and choose $\alpha$ and $\beta$ such that

$$
\begin{aligned}
& \beta^{2}+(q-1) \beta+\frac{B}{4}=0, \\
& (\alpha+\beta)(\alpha+\beta+p+q-1)+\frac{C}{4}=0 .
\end{aligned}
$$

Then, by the change of variable $\zeta=\frac{1}{\cosh ^{2} t}$, the ordinary differential equation

$$
\left\{\frac{\mathrm{d}^{2}}{\mathrm{~d} t^{2}}+((2 p-1) \tanh t+(2 q-1) \operatorname{coth} t) \frac{\mathrm{d}}{\mathrm{d} t}+\left(\frac{A}{\cosh ^{2} t}+\frac{B}{\sinh ^{2} t}+C\right)\right\} f(t)=0
$$

for a unknown function $f(t)$ on $t>0$ is transformed to the Gaussian hypergeometric equation

$$
\zeta(1-\zeta) F^{\prime \prime}(\zeta)+\{c-(a+b+1) \zeta\} F^{\prime}(\zeta)-a b F(\zeta)=0
$$

with

$$
(a+b, a b, c)=\left(-2 \alpha-p+1, \alpha^{2}+(p-1) \alpha-\frac{A}{4},-(p+q+2 \alpha+2 \beta-2)\right),
$$

for the unknown function $F(\zeta)=\left(\cosh ^{2} t\right)^{-\alpha}\left(\sinh ^{2} t\right)^{-\beta} f(t)$ on $0<\zeta<1$.

Proof. A direct computation.

Let us apply this lemma to our equation (5.17) taking $A=4 \kappa(\kappa+p-1), B=0$ and $C=\rho_{0}^{2}-s^{2}$. Then $\beta=0, \alpha=-\frac{1}{2}\left(\rho_{0}+s\right)$ satisfies (5.18) and (5.19). We can easily find

$$
(a, b, c)=\left(\frac{s+p+q-1}{2}+\kappa, \frac{s-p+q+1}{2}-\kappa, s+1\right) .
$$

satisfies (5.21). The condition (5.16) is equivalent to the condition that $F(\zeta)=$ $(\cosh t)^{s+\rho_{0}} \phi_{\kappa}(t)$ should be bounded as $\zeta \rightarrow+0$. The solution of (5.20) given by the hypergeometric series

$$
F(\zeta)={ }_{2} F_{1}(a, b ; c ; \zeta)=\frac{\Gamma(c)}{\Gamma(a) \Gamma(b)} \sum_{n=0}^{\infty} \frac{\Gamma(a+n) \Gamma(b+n)}{\Gamma(c+n) n !} \zeta^{n}, \quad(|\zeta|<1)
$$

meets this requirement. Thus we have shown that the function

$$
\phi_{\kappa}(t)=C_{\kappa}(\cosh t)^{-\left(s+\rho_{0}\right)}{ }_{2} F_{1}\left(\frac{s+p+q-1}{2}+\kappa, \frac{s-p+q+1}{2}-\kappa ; s+1 ; \frac{1}{\cosh ^{2} t}\right)
$$

with a constant $C_{\kappa} \in \mathbb{C}$ satisfies all the conditions we require except (5.15). For this function to satisfy (5.15), the constant $C_{\kappa}$ has to be

$$
C_{\kappa}=\frac{\Gamma\left(\frac{s+p+q-1}{2}+\kappa\right) \Gamma\left(\frac{s-p+q+1}{2}-\kappa\right)}{\Gamma(s+1) \Gamma(q-1)}
$$


by a formula in $[19, \mathrm{p} .49]$. This completes the proof that the function given by (5.1) satisfies all the conditions in Theorem 18.

5.2.2. The uniqueness. Let us prove the uniqueness of $\varphi_{s}^{(d)}\left(s \in D^{(d)}\right)$ satisfying the conditions (i) to (v) in Theorem 18. For that, take another family $\psi_{s}^{(d)}(s \in$ $\left.D^{(d)}\right)$ with the same properties as $\varphi_{s}^{(d)}$. Fix $s \in \mathbb{C}$ such that $\operatorname{Re}(s)>\rho_{0}$ and consider the difference $f_{s}(g)=\varphi_{s}^{(d)}(g)-\psi_{s}^{(d)}(g)$, which defines a $(d, d)$-current on $G / K$ by Lemma 22 and satisfies the differential equation $\triangle f_{s}=\left(\rho_{0}^{2}-s^{2}\right) f_{s}$ by Proposition 23. Since $\triangle$ is an elliptic differential operator, $f_{s}$ is automatically real analytic on $G / K$.

Let $0<\epsilon<R$. We obtain $\mathcal{D}_{t} f_{s}\left(a_{t}\right)=\left(\rho_{0}^{2}-s^{2}\right) f_{s}\left(a_{t}\right)(\forall t>0)$ from $\triangle f_{s}=$ $\left(\rho_{0}^{2}-s^{2}\right) f_{s}$ by Proposition 14; thus, Lemma 15 yields the identity

$$
\left(s^{2}-\bar{s}^{2}\right) \int_{\epsilon}^{R}\left\|f_{s}\left(a_{t}\right)\right\|^{2} \varrho(t) \mathrm{d} t=\mathfrak{F}(\epsilon)-\mathfrak{F}(R)
$$

with

$$
\mathfrak{F}(t)=\varrho(t)\left\{\left(f_{s}\left(a_{t}\right) \mid \frac{\mathrm{d}}{\mathrm{d} t} f_{s}\left(a_{t}\right)\right)-\left(\frac{\mathrm{d}}{\mathrm{d} t} f_{s}\left(a_{t}\right) \mid f_{s}\left(a_{t}\right)\right)\right\} .
$$

Since $f_{s}$ is real analytic on $G / K$, the function $t \mapsto f_{s}\left(a_{t}\right)$ is smooth on $\mathbb{R}$. Noting this, the limit of $\mathfrak{F}(\epsilon)$ as $\epsilon \rightarrow+0$ is easily computed as

$$
\lim _{\epsilon \rightarrow+0} \mathfrak{F}(\epsilon)=0 .
$$

By (5.2), the function $t \mapsto f_{s}\left(a_{t}\right)$ as well as its derivative $\frac{d}{d t} f_{s}\left(a_{t}\right)$ is majorized by $O\left(e^{-\left(\operatorname{Re}(s)+\rho_{0}\right) t}\right)$ for large $t$. Hence $\mathfrak{F}(R)=O\left(\varrho(t) e^{-\left(\operatorname{Re}(s)+\rho_{0}\right) t}\right)=O\left(e^{-\left(\operatorname{Re}(s)-\rho_{0}\right) t}\right)$, which implies

$$
\lim _{R \rightarrow+\infty} \mathfrak{F}(R)=0 .
$$

By (5.22), (5.23) and (5.24), we obtain

$$
\left(s^{2}-\bar{s}^{2}\right) \int_{0}^{+\infty}\left\|f_{s}\left(a_{t}\right)\right\|^{2} \varrho(t) \mathrm{d} t=0 .
$$

This identity yields $\int_{0}^{+\infty}\left\|f_{s}\left(a_{t}\right)\right\|^{2} \varrho(t) \mathrm{d} t=0$ as long as $s^{2} \notin \mathbb{R}$. Since the function $\left\|f_{s}\left(a_{t}\right)\right\|^{2} \varrho(t)$ on $t \geqslant 0$ is continuous and non-negative, the vanishing of its integral implies the vanishing of the function itself: $\left\|f_{s}\left(a_{t}\right)\right\|^{2} \varrho(t)=0(\forall t \geqslant 0)$. Noting $\varrho(t)>0(\forall t>0)$, we consequently obtain $f_{s}\left(a_{t}\right)=0(\forall t \geqslant 0)$ under the assumption $\operatorname{Re}(s)>\rho_{0}, s^{2} \notin \mathbb{R}$. By the decomposition $G=H\left\{a_{t} \mid t \geqslant 0\right\} K$ and by the equivariance $f_{s}(h g k)=\tau(k)^{-1} f_{s}(g)(\forall(h, k) \in H \times K)$, the value $f_{s}(g)$ 
has to be zero for all $g \in G$ and for all $s$ such that $s^{2} \notin \mathbb{R}, \operatorname{Re}(s)>\rho_{0}$. Using the condition (ii), we finally conclude $\varphi_{s}^{(d)}(g)=\psi_{s}^{(d)}(g)(\forall g \in G-H K)$ for all $s \in D^{(d)}$ by analytic continuation.

5.3. A finer form of small-time asymptotic. By the explicit formula (5.1), we obtain a finer small-time asymptotic than (5.3).

Proposition 25. There exists a unique family $\mathrm{c}_{\alpha}(s)(0 \leqslant \alpha \leqslant q-2)$ of tensors in $\left(\bigwedge^{d, d} \mathfrak{p}_{\mathbb{C}}^{*}\right)^{H \cap K}$ such that the following properties hold.

(1) There exist $\left(\bigwedge \mathfrak{p}_{\mathbb{C}}^{*}\right)^{H \cap K}$-valued holomorphic functions $\mathrm{P}(s, z)$ and $\mathrm{Q}(s, z)$ on $D^{(d)} \times\{|z|<1\}$ such that

$$
\begin{gathered}
\varphi_{s}^{(d)}\left(a_{t}\right)=\sum_{\alpha=0}^{q-2} \mathrm{c}_{\alpha}(s) z^{-q+\alpha+1}+\mathrm{P}(s, z)+\log z \mathrm{Q}(s, z), \\
\left(s \in D^{(d)}, z=\tanh ^{2} t \in(0,1)\right) .
\end{gathered}
$$

(2) We have $\mathrm{c}_{0}(s)=\Lambda^{q-d}\left(* \mathrm{vol}_{\mathfrak{p} \cap \mathfrak{h}}\right)$, and $\mathrm{c}_{\alpha}(s)$ satisfies the recurrence relation:

$$
\begin{aligned}
4 \alpha(q-\alpha-1) \mathrm{c}_{\alpha}(s)= & \sum_{\kappa=0}^{\alpha-1}\left\{\tau\left(\Omega_{\mathfrak{k}}\right)+(\alpha-\kappa)\left(\rho_{0}^{2}-s^{2}\right)\right. \\
& \left.\quad-4\left(\rho_{0}-1\right)(q-\kappa-1)\right\} \mathrm{c}_{\kappa}(s), \quad(0<\alpha \leqslant q-2) .
\end{aligned}
$$

(3) For $\alpha, r \in \mathbb{N}$ such that $0 \leqslant \alpha \leqslant \inf (r-1, q-2)$,

$$
\left(\frac{-1}{2 s} \frac{\mathrm{d}}{\mathrm{d} s}\right)^{r} \mathrm{c}_{\alpha}(s)=0 .
$$

Proof. The formula (5.25) follows readily from our explicit formula (5.1) combined with a property of the hypergeometric function [19, p.49].

The recurrence formula (5.26) is obtained first by substituting the expansion (5.25) to the equation (5.4) and then by equating the coefficient of $z^{-q+\alpha-1}$ in the left-hand side with 0 .

By (2), it is obvious that $\mathrm{c}_{\alpha}(s)(0 \leqslant \alpha \leqslant q-2)$ is a polynomial function in $s \in \mathbb{C}$ such that $\mathrm{c}_{\alpha}(s)=\mathrm{c}_{\alpha}(-s)$ and $\operatorname{deg} c_{\alpha}(s)=2 \alpha$. Hence $\mathrm{c}_{\alpha}(s)=\mathrm{b}\left(s^{2}\right)$ with some polynomial $\mathrm{b}(t)$ of degree $\alpha$. If we set $t=s^{2}$, then $\left(\frac{-1}{2 s} \frac{d}{d s}\right)^{r} \mathrm{c}_{\alpha}(s)=\left(-\frac{d}{d t}\right)^{r} \mathrm{~b}(t)=$ 0 since $r>\alpha$. This shows (3). 
5.4. A differential relation. The functions $\phi_{s}=\varphi_{s}^{(q-1)}=\Lambda \varphi_{s}^{(q)}$ and $\psi_{s}=\varphi_{s}^{(q)}$ are of particular importance in our investigation of the modular cycles arising from $H$. They are related by the simple formula:

Theorem 26. Let $s \in D^{(q)}$. Then we have

$$
\partial \bar{\partial} \phi_{s}(g)=\frac{-\sqrt{-1}\left(s^{2}-\rho_{0}^{2}\right)}{2} \psi_{s}(g), \quad g \in G-H K .
$$

In order to prove this, it suffices to show that $\tilde{\psi}_{s}=2 \sqrt{-1}\left(s^{2}-\rho_{0}^{2}\right)^{-1} \partial \bar{\partial} \phi_{s}$ has the properties (i) to (v) for $d=q$ which characterizes the function $\psi_{s}$ by Theorem 18.

The property (i) for $\tilde{\psi}_{s}$ is obvious by definition. The property (ii) is also obvious by the explicit formula (5.1) and Proposition 16. The property (iii) for $\tilde{\psi}_{s}$ follows from the corresponding equation for $\phi_{s}$ because $\Omega_{\mathfrak{g}}$ is commutative with the operators $\partial, \bar{\partial}$. The large-time asymptotic of $\tilde{\psi}_{s}\left(a_{t}\right)$ is easy to prove by Proposition 16, because (5.2) shows that any derivative $\frac{d^{j}}{d t^{j}} \phi_{s}\left(a_{t}\right)$ is majorized by $O\left(e^{-\left(\operatorname{Re}(s)+\rho_{0}\right) t}\right)$ for large $t$. It remains to show

$$
\lim _{t \rightarrow+0} t^{2(q-1)} \partial \bar{\partial} \phi_{s}\left(a_{t}\right)=\frac{-\sqrt{-1}\left(s^{2}-\rho_{0}^{2}\right)}{2} * \operatorname{vol}_{\mathfrak{p} \cap \mathfrak{h}} .
$$

The rest of this subsection is devoted to the proof of (5.27).

From the equation $\Omega_{\mathfrak{g}} \phi_{s}(g)=\left(s^{2}-\rho_{0}^{2}\right) \phi_{s}(g)(g \in G-H K)$ and Proposition 14, we have

$$
\frac{\mathrm{d}^{2}}{\mathrm{~d} t^{2}} \phi(t)=-((2 p-1) \tanh t+(2 q-1) \operatorname{coth} t) \frac{\mathrm{d}}{\mathrm{d} t} \phi(t)+\frac{4 \mathcal{S}_{\mathrm{E}, \mathfrak{q}}}{\cosh ^{2} t} \phi(t)+\left(s^{2}-\rho_{0}^{2}\right) \phi(t)
$$

with $\phi(t)=\phi_{s}\left(a_{t}\right)$. By this, we eliminate the second derivative $\phi^{\prime \prime}(t)$ in the formula $\varepsilon_{t} \phi(t)$ in Proposition 16 to obtain

$$
\begin{aligned}
& \partial \bar{\partial} \phi_{s}\left(a_{t}\right) \\
& =\left\{-\frac{1}{4}((2 p-1) \tanh t+(2 q-1) \operatorname{coth} t) e\left(\omega_{0} \wedge \bar{\omega}_{0}\right)+\frac{1}{2}(\mathcal{A} \tanh t+\mathcal{B} \operatorname{coth} t)\right\} \phi^{\prime}(t) \\
& +\left\{\frac{e\left(\omega_{0} \wedge \bar{\omega}_{0}\right)}{\cosh ^{2} t} \mathcal{S}_{\mathfrak{k}, \mathfrak{q}}+\mathcal{P}_{-} \mathcal{P}_{+} \tanh ^{2} t+\mathcal{R}_{-} \mathcal{R}_{+} \operatorname{coth}^{2} t+\mathcal{C}+\frac{s^{2}-\rho_{0}^{2}}{4} e\left(\omega_{0} \wedge \bar{\omega}_{0}\right)\right\} \phi(t) .
\end{aligned}
$$

By the coordinate $z=\tanh ^{2} t$, this becomes

$$
\begin{aligned}
& \partial \bar{\partial} \phi_{s}\left(a_{t}\right) \\
& =(1-z)\left\{\frac{-1}{2}((2 p-1) z+2 q-1) e\left(\omega_{0} \wedge \bar{\omega}_{0}\right)+(\mathcal{A} z+\mathcal{B})\right\} \frac{\mathrm{d} \phi}{\mathrm{d} z}(z) \\
& +\left\{(1-z) e\left(\omega_{0} \wedge \bar{\omega}_{0}\right) \mathcal{S}_{\mathfrak{k}, \mathfrak{q}}+\mathcal{P}_{-} \mathcal{P}_{+} z+\mathcal{R}_{-} \mathcal{R}_{+} z^{-1}+\mathcal{C}+\frac{s^{2}-\rho_{0}^{2}}{4} e\left(\omega_{0} \wedge \bar{\omega}_{0}\right)\right\} \phi(z) .
\end{aligned}
$$


Using this and (5.25), we can compute the first two terms of the singular part of $\partial \bar{\partial} \phi_{s}\left(a_{t}\right)$ in the $z$-expansion, which is of the form

$$
\partial \bar{\partial} \phi_{s}\left(a_{t}\right)=\sum_{j=1}^{q} \frac{\mathrm{a}_{q-j}(s)}{z^{j}}+\mathrm{P}_{1}(s, z)+(\log z) \mathrm{P}_{2}(s, z)
$$

with some polynomial functions $\mathrm{a}_{j}(s)$ and some holomorphic functions $\mathrm{P}_{1}(s, z)$, $\mathrm{P}_{2}(s, z)$. We are interested in $\mathrm{a}_{0}(s)$ and $\mathrm{a}_{1}(s)$. A short computation yields

$$
\begin{aligned}
\mathrm{a}_{0}(s)= & \left\{-(q-1)\left(-\frac{2 q-1}{2} e\left(\omega_{0} \wedge \bar{\omega}_{0}\right)+\mathcal{B}\right)+\mathcal{R}_{-} \mathcal{R}_{+}\right\} \mathrm{c}_{0}(s), \\
\mathrm{a}_{1}(s)= & \left\{-(q-1)\left(-\frac{2 p-1}{2} e\left(\omega_{0} \wedge \bar{\omega}_{0}\right)+\mathcal{A}\right)+(q-1)\left(-\frac{2 q-1}{2} e\left(\omega_{0} \wedge \bar{\omega}_{0}\right)+\mathcal{B}\right)\right. \\
& \left.+e\left(\omega_{0} \wedge \bar{\omega}_{0}\right) \mathcal{S}_{\mathfrak{k}, \mathfrak{q}}+\mathcal{C}+\frac{s^{2}-\rho_{0}^{2}}{4} e\left(\omega_{0} \wedge \bar{\omega}_{0}\right)\right\} \mathrm{c}_{0}(s) \\
+ & \left\{\begin{array}{l}
\left\{-\mathcal{R} \_\mathcal{R}_{+}+(q-2)\left(-\frac{2 q-1}{2} e\left(\omega_{0} \wedge \bar{\omega}_{0}\right)+\mathcal{B}\right)\right\} \mathrm{c}_{1}(s), \quad(q>2), \\
\left.-\frac{2 q-1}{2} e\left(\omega_{0} \wedge \bar{\omega}_{0}\right)+\mathcal{B}\right\} \mathrm{Q}(s, 0), \quad(q=2) .
\end{array}\right.
\end{aligned}
$$

By (5.26), we have

$$
\begin{aligned}
(q-1) ! \mathrm{c}_{0}(s) & =\gamma_{p p}^{q-1}=\left(\frac{\sqrt{-1}}{2}\right)^{q-1}\left(\omega_{p 1} \wedge \bar{\omega}_{p 1}+\eta_{\mathfrak{q}}\right)^{q-1}, \\
(q-1) !(q-2) \mathrm{c}_{1}(s) & =\left\{\frac{s^{2}-\rho_{0}^{2}}{4}+(q-1)\left(\rho_{0}-1\right)\right\} \gamma_{p p}^{q-1}+\mathcal{S}_{\mathfrak{k}, \mathfrak{q}} \gamma_{p p}^{q-1}, \quad(q>2), \\
\mathrm{Q}(s, 0) & =\frac{s^{2}-\rho_{0}^{2}}{4} \gamma_{p p}+\mathcal{S}_{\mathfrak{k}, \mathfrak{q}} \gamma_{p p}, \quad(q=2)
\end{aligned}
$$

with $\eta_{\mathfrak{q}}=\sum_{j=1}^{q-1} \omega_{p, j+1} \wedge \bar{\omega}_{p, j+1}$.

\section{Lemma 27.}

$$
\left\{\frac{2 q-1}{2} e\left(\omega_{0} \wedge \bar{\omega}_{0}\right)-\mathcal{B}\right\} \gamma_{p p}^{q-1}=\left\{(q-1) \omega_{p 1} \wedge \bar{\omega}_{p 1}-\eta_{\mathfrak{q}}\right\} \wedge \gamma_{p p}^{q-1}=0
$$


Proof. Since $\gamma_{p p}$ is $H \cap K$-invariant, $\mathcal{R}_{ \pm} \gamma_{p p}^{q-1}=0$. Hence we have

$$
\begin{aligned}
& \left\{\frac{2 q-1}{2} e\left(\omega_{0} \wedge \bar{\omega}_{0}\right)-\mathcal{B}\right\} \gamma_{p p}^{q-1} \\
& =\left\{(q-1) \omega_{p 1} \wedge \bar{\omega}_{p 1}-\eta_{\mathfrak{q}}\right\} \wedge \gamma_{p p}^{q-1} \\
& =\left(\frac{\sqrt{-1}}{2}\right)^{q-1}\left\{(q-1) \omega_{p 1} \wedge \bar{\omega}_{p 1}-\eta_{\mathfrak{q}}\right\} \wedge\left(\omega_{p 1} \wedge \bar{\omega}_{p 1}+\eta_{\mathfrak{q}}\right)^{q-1} \\
& =\left(\frac{\sqrt{-1}}{2}\right)^{q-1}\left\{(q-1) \omega_{p 1} \wedge \bar{\omega}_{p 1}-\eta_{\mathfrak{q}}\right\} \wedge\left\{\eta_{\mathfrak{q}}^{q-1}+(q-1) \omega_{p 1} \wedge \bar{\omega}_{p 1} \wedge \eta_{\mathfrak{q}}^{q-2}\right\} \\
& =-\left(\frac{\sqrt{-1}}{2}\right)^{q-1} \eta_{\mathfrak{q}}^{q} \\
& =0 .
\end{aligned}
$$

Since $\mathrm{c}_{0}(s)$ and $\mathrm{c}_{1}(s)$ are $H \cap K$-invariant (Proposition 25),

$$
\mathcal{R}_{ \pm} \mathrm{C}_{0}(s)=\mathcal{R}_{ \pm} \mathrm{c}_{1}(s)=0 .
$$

Therefore, by Lemma $27, \mathrm{a}_{0}(s)=0$.

By $(4.5),(4.6),(4.7)$ and Lemma 27 , noting that $\mathcal{R}_{+} \mathrm{c}_{1}(s)=0$, we have

$$
\begin{aligned}
(q & -1) ! \mathrm{a}_{1}(s) \\
= & \left\{-(q-1)\left(-\frac{2 p-1}{2} e\left(\omega_{0} \wedge \bar{\omega}_{0}\right)+\mathcal{A}\right)+e\left(\omega_{0} \wedge \bar{\omega}_{0}\right) \mathcal{S}_{\mathfrak{k}, \mathfrak{q}}+\mathcal{C}+\frac{s^{2}-\rho_{0}^{2}}{4} e\left(\omega_{0} \wedge \bar{\omega}_{0}\right)\right\} \gamma_{p p}^{q-1} \\
& +\left\{-\frac{2 q-1}{2} e\left(\omega_{0} \wedge \bar{\omega}_{0}\right)+\mathcal{B}\right\} \mathcal{S}_{\mathfrak{k}, \mathfrak{q}} \gamma_{p p}^{q-1} \\
= & (q-1)\left\{(p-1) e\left(\omega_{p 1} \wedge \bar{\omega}_{p 1}\right)+e\left(\bar{\omega}_{p 1}\right) \mathcal{P}_{-}-e\left(\omega_{p 1}\right) \mathcal{P}_{+}-e\left(\eta_{\mathfrak{h}}\right)\right\} \gamma_{p p}^{q-1} \\
& +\left\{e\left(\omega_{p 1} \wedge \bar{\omega}_{p 1}\right) \mathcal{S}_{\mathfrak{k}, \mathfrak{q}}+e\left(\omega_{p 1}\right) \mathcal{P}_{+}+\mathcal{R}_{-} \mathcal{P}_{+}\right\} \gamma_{p p}^{q-1} \\
& +\left\{-(q-1) \omega_{p 1} \wedge \bar{\omega}_{p 1}+\eta_{\mathfrak{q}}\right\} \wedge \mathcal{S}_{\mathfrak{k}, \mathfrak{q}} \gamma_{p p}^{q-1} \\
& +\frac{s^{2}-\rho_{0}^{2}}{4} e\left(\omega_{p 1} \wedge \bar{\omega}_{p 1}\right) \gamma_{p p}^{q-1} .
\end{aligned}
$$

Lemma 28. Set $B=-\sum_{i=1}^{p-1} \gamma_{p i} \wedge \gamma_{i p}$. Then

$$
\begin{aligned}
e\left(\omega_{p 1} \wedge \bar{\omega}_{p 1}\right) \gamma_{p p}^{q-1} & =\frac{2}{q \sqrt{-1}} \gamma_{p p}^{q}, \\
\mathcal{P}_{-} \gamma_{p p}^{q-1} & =(q-1) \sum_{i=1}^{p-1} \omega_{i 1} \wedge \gamma_{p i} \wedge \gamma_{p p}^{q-2}, \\
\mathcal{P}_{+} \gamma_{p p}^{q-1} & =(q-1) \sum_{i=1}^{p-1} \bar{\omega}_{i 1} \wedge \gamma_{i p} \wedge \gamma_{p p}^{q-2},
\end{aligned}
$$


The Secondary Spherical Functions and Automorphic Green ... 1011

$$
\begin{aligned}
\mathcal{R}_{-} \mathcal{P}_{+} \gamma_{p p}^{q-1}= & (q-1)\left\{\frac{-2}{\sqrt{-1}} B \wedge \gamma_{p p}^{q-2}-\sum_{i=1}^{p-1} \omega_{p 1} \wedge \bar{\omega}_{i 1} \wedge \gamma_{i p} \wedge \gamma_{p p}^{q-2}\right\}, \\
\mathcal{S}_{\mathfrak{k}, \mathfrak{q}} \gamma_{p p}^{q-1}= & (q-1)\left\{-(p-1) \gamma_{p p}^{q-1}+\sum_{i=1}^{p-1} \gamma_{i i} \wedge \gamma_{p p}^{q-2}-(q-2) B \wedge \gamma_{p p}^{q-3}\right\}, \\
\left(\frac{\sqrt{-1}}{2}\right)^{q-1} \frac{1}{q} \mathcal{S}_{\mathfrak{k}, \mathfrak{q}} \eta_{\mathfrak{q}}^{q}= & \frac{2}{\sqrt{-1}}\left\{-(p-1) \gamma_{p p}^{q}+\sum_{i=1}^{p-1} \gamma_{i i} \wedge \gamma_{p p}^{q-1}-(q-1) B \wedge \gamma_{p p}^{q-2}\right\} \\
& -\omega_{p 1} \wedge \bar{\omega}_{p 1} \wedge \mathcal{S}_{\mathfrak{k}, \mathfrak{q}} \gamma_{p p}^{q-1}-e\left(\eta_{\mathfrak{h}}\right) \gamma_{p p}^{q-1}+(p-1) \omega_{p 1} \wedge \bar{\omega}_{p 1} \wedge \gamma_{p p}^{q-1} \\
& +(q-1) \bar{\omega}_{p 1} \wedge\left(\sum_{i=1}^{p-1} \omega_{i 1} \wedge \gamma_{p i}\right) \wedge \gamma_{p p}^{q-2} \\
& -(q-1) \omega_{p 1} \wedge\left(\sum_{i=1}^{p-1} \bar{\omega}_{i 1} \wedge \gamma_{i p}\right) \wedge \gamma_{p p}^{q-2} .
\end{aligned}
$$

Proof. A direct computation with the aid of Lemma 6 .

By this lemma, a direct computation yields the identity

$$
\begin{aligned}
& (q-1)\left\{(p-1) e\left(\omega_{p 1} \wedge \bar{\omega}_{p 1}\right)+e\left(\bar{\omega}_{p 1}\right) \mathcal{P}_{-}-e\left(\omega_{p 1}\right) \mathcal{P}_{+}-e\left(\eta_{\mathfrak{h}}\right)\right\} \gamma_{p p}^{q-1} \\
& \quad+\left\{e\left(\omega_{p 1} \wedge \bar{\omega}_{p 1}\right) \mathcal{S}_{\mathfrak{k}, \mathfrak{q}}+e\left(\omega_{p 1}\right) \mathcal{P}_{+}+\mathcal{R}_{-} \mathcal{P}_{+}\right\} \gamma_{p p}^{q-1} \\
& \quad+\left\{-(q-1) \omega_{p 1} \wedge \bar{\omega}_{p 1}+\eta_{\mathfrak{q}}\right\} \wedge \mathcal{S}_{\mathfrak{k}, \mathfrak{q}} \gamma_{p p}^{q-1} \\
& =\left(\frac{\sqrt{-1}}{2}\right)^{q-1} \frac{q-1}{q} \mathcal{S}_{\mathfrak{k}, \mathfrak{q}} \eta_{\mathfrak{q}}^{q},
\end{aligned}
$$

which simplifies (5.29) considerably:

$$
\begin{aligned}
(q-1) ! \mathrm{a}_{1}(s) & =\left(\frac{\sqrt{-1}}{2}\right)^{q-1} \frac{q-1}{q} \mathcal{S}_{\mathfrak{k}, \mathfrak{q}} \eta_{\mathfrak{q}}^{q}+\frac{s^{2}-\rho_{0}^{2}}{4} e\left(\omega_{p 1} \wedge \bar{\omega}_{p 1}\right) \gamma_{p p}^{q-1} \\
& =\frac{s^{2}-\rho_{0}^{2}}{2 q \sqrt{-1}} \gamma_{p p}^{q} \\
& =\frac{s^{2}-\rho_{0}^{2}}{2 \sqrt{-1}}(q-1) ! * \operatorname{vol}_{\mathfrak{p} \cap \mathfrak{h}},
\end{aligned}
$$

where the second equality follows from $\eta_{\mathfrak{q}}^{q}=0$ combined with the first formula in Lemma 28 and the third equality follows from Lemma 7. Summing up, we obtain

$$
\mathrm{a}_{0}(s)=0, \quad \mathrm{a}_{1}(s)=\frac{-\sqrt{-1}\left(s^{2}-\rho_{0}^{2}\right)}{2} * \operatorname{vol}_{\mathfrak{p} \cap \mathfrak{h}} .
$$

This, combined with (5.28), implies the desired limit (5.27). As explained at the begining of this subsection, this completes the proof of Theorem 26 . 


\section{Poincaré SERIES}

From now on, we fix a $\mathbb{Q}$-structure on $G$ such that the involution $\sigma$ defining $H$ is defined over $\mathbb{Q}$. Let $\Gamma$ be an arithmetic subgroup in $G$. Then $H$ is a $\mathbb{Q}$-subgroup of $G$ and the intersection $\Gamma \cap H$ is an arithmetric subgroup of $H$. The quotient spaces $\Gamma \backslash G$ and $\Gamma \cap H \backslash H$ have finite invariant volumes. For simplicity we set $\Gamma_{H}=\Gamma \cap H$ and $K_{H}=H \cap K$, and suppose that $\Gamma$ is neat. Then, the Kähler manifold structures on the discrete quotients $\Gamma_{H} \backslash H / K_{H}$ and $\Gamma \backslash G / K$ are pushed down from those on their universal coverings $H / K_{H}$ and $G / K$. Moreover, $\Gamma H$ is a closed subset of $G$ and the inclusion $\Gamma \cap H \backslash H \hookrightarrow \Gamma \backslash G$ has the closed image.

6.1. Currents defined by Poincaré series. Let $\varphi_{s}^{(d)}\left(s \in D^{(d)}\right)$ be the secondary spherical function of bidegree $(d, d)$ constructed in Theorem 18. For $r \in \mathbb{N}$, we define an auxiliary function $\varphi_{s, r}^{(d)}$ by

$$
\varphi_{s, r}^{(d)}(g)=\frac{1}{r !}\left(\frac{-1}{2 s} \frac{\mathrm{d}}{\mathrm{d} s}\right)^{r} \varphi_{s}^{(d)}(g), \quad\left(s \in D^{(d)}, g \in G-H K\right) .
$$

Let us consider the Poincaré series

$$
\Phi_{s, r}^{(d)}(g)=\frac{\Gamma(q-1)}{\pi^{q}} \sum_{\gamma \in \Gamma_{H} \backslash \Gamma} \varphi_{s, r}^{(d)}(\gamma g)
$$

for $(s, g)$ belonging to the set $\left\{s \in \mathbb{C} \mid \operatorname{Re}(s)>\rho_{0}\right\} \times(G-\Gamma H K)$, where the series is $L^{1}$-convergent as the next proposition shows. Note $\operatorname{Re}(s)>\rho_{0}$ is contained in the domain $D^{(d)}$.

Proposition 29. Suppose $\operatorname{Re}(s)>\rho_{0}$. Then

$$
\int_{\Gamma \backslash G} \sum_{\gamma \in \Gamma_{H} \backslash \Gamma}\left\|\varphi_{s, r}^{(d)}(g)\right\| \mathrm{d} g<+\infty .
$$

In particular, the measurable function $\Phi_{s, r}^{(d)}(g)$ on $\Gamma \backslash G / K$ is integrable.

Proof. By the integration formula (2.3), the integral in question equals

$$
\int_{\Gamma_{H} \backslash G}\left\|\varphi_{s, r}^{(d)}(g)\right\| \mathrm{d} g=\int_{\Gamma_{H} \backslash H} \mathrm{~d} h \int_{K} \mathrm{~d} k \int_{0}^{+\infty}\left\|\varphi_{s, r}^{(d)}\left(h a_{t} k\right)\right\| \varrho(t) \mathrm{d} t .
$$

Since $\varphi_{s, r}^{(d)}(h g k)=\tau(k)^{-1} \varphi_{s}^{(d)}(g)(\forall(h, k) \in H \times K)$, and since $\tau$ is unitary, the integral over $\Gamma_{H} \backslash H$ yields the factor $\operatorname{vol}\left(\Gamma_{H} \backslash H\right)$, which is finite as we remarked above, and the integral over $K$ yields the factor 1 . To complete the proof, it suffices to show the convergence of the integral $\int_{0}^{+\infty}\left\|\varphi_{s, r}^{(d)}\left(a_{t}\right)\right\| \varrho(t) \mathrm{d} t$. 
By applying the differential operator $\left(\frac{-1}{2 s} \frac{\partial}{\partial s}\right)^{r}$ to the formula (5.25), noting Proposition 25 (3), we obtain the estimation $\left\|\varphi_{s, r}^{(d)}\left(a_{t}\right)\right\|=O\left(t^{-2 q+2 r+2}\right)$ on $(0,1]$. Hence the function $\left\|\varphi_{s, r}^{(d)}\left(a_{t}\right)\right\|$ is majorized by $O\left(\varrho(t) t^{-2 q+2 r+2}\right)=O\left(t^{2 r+1}\right)$ on $(0,1]$, especially integrable there.

By applying $\left(\frac{-1}{2 s} \frac{\partial}{\partial s}\right)^{r}$ to the formula (5.2), we easily see that $\varphi_{s, r}^{(d)}\left(a_{t}\right)$ is still majorized by $O\left(e^{-\left(\operatorname{Re}(s)+\rho_{0}\right) t}\right)$ on the interval $[1,+\infty)$. Hence $\left\|\varphi_{s}^{(d)}\left(a_{t}\right)\right\| \varrho(t)=$ $O\left(e^{-\left(\operatorname{Re}(s)-\rho_{0}\right) t}\right)$, which implies the convergence of the improper integral over $[1,+\infty)$ when $\operatorname{Re}(s)>\rho_{0}$. This completes the proof.

Therefore the measurable $(d, d)$-form $\Phi_{s, r}^{(d)}$ on $\Gamma \backslash G / K$ yields a current, denoted by the same notation $\Phi_{s, r}^{(d)}$, by the integration:

$$
\left\langle\Phi_{s, r}^{(d)}, \alpha\right\rangle=\int_{\Gamma \backslash G / K} \Phi_{s, r}^{(d)} \wedge \alpha, \quad\left(\forall \alpha \in \mathcal{A}_{\mathrm{c}}(\Gamma \backslash G / K)\right) .
$$

6.2. The Poisson equation. Let $C_{H}^{\Gamma}: \Gamma_{H} \backslash H / K_{H} \rightarrow \Gamma \backslash G / K$ be the holomorphic map obtained from the inclusion $H / K_{H} \hookrightarrow G / K$ by passing to the discrete quotients. Since we assume that $\Gamma$ is neat, the map $C_{H}^{\Gamma}$ is an inclusion and its image is a closed complex analytic subset of $\Gamma \backslash G / K$. The integration

$$
\left\langle\delta_{C_{H}^{\Gamma}}, \alpha\right\rangle=\int_{\Gamma_{H} \backslash H / K_{H}}\left(C_{H}^{\Gamma}\right)^{*} \alpha, \quad \alpha \in \mathcal{A}_{\mathrm{c}}(\Gamma \backslash G / K)
$$

defines a $(q, q)$-current on $\Gamma \backslash G / K$.

Lemma 30. For $\alpha \in \mathcal{A}_{\mathrm{c}}(\Gamma \backslash G / K)$, we have

$$
\left\langle\Lambda^{q-d} \delta_{C_{H}^{\Gamma}}, * \bar{\alpha}\right\rangle=\left(\Lambda^{q-d}\left(* \operatorname{vol}_{\mathfrak{p} \cap \mathfrak{h}}\right) \mid \alpha^{\Gamma_{H} \backslash H}(e)\right),
$$

where

$$
\alpha^{\Gamma_{H} \backslash H}(g)=\int_{\Gamma_{H} \backslash H} \alpha(h g) \mathrm{d} h .
$$

Proof. This is proved by a similar way to Lemma 3. See also [32, Proposition 6.1].

For a while, we use the simpler notation $C$ for $C_{H}^{\Gamma}$. Our currents $\Phi_{s, r}^{(d)}$ satisfy the generalized Poisson equation: 


\section{Proposition 31.}

$$
\left(\triangle+s^{2}-\rho_{0}^{2}\right)^{r+1} \Phi_{s, r}^{(d)}=4 \Lambda^{q-d} \delta_{C}
$$

for $\operatorname{Re}(s)>\rho_{0}, r \in \mathbb{N}$.

Proof. The proof is similar to the local counterpart Proposition 23. For $\alpha \in$ $\mathcal{A}_{\mathrm{C}}(\Gamma \backslash G / K)$, set $I_{r}(\alpha)=\left\langle\left(\triangle+s^{2}-\rho_{0}^{2}\right)^{r+1} \Phi_{s, r}^{(d)}, * \bar{\alpha}\right\rangle$. Then it suffices to prove $I_{r}(\alpha)=I_{r-1}(\alpha)(\forall r \geqslant 1)$ and $I_{0}(\alpha)=\left\langle 4 \Lambda^{q-d} \delta_{C}, * \alpha\right\rangle$. We have

$$
\begin{aligned}
I_{r}(\alpha) & =\left\langle\Phi_{s, r}^{(d)},\left(\triangle+s^{2}-\rho_{0}^{2}\right)^{r+1} * \bar{\alpha}\right\rangle \\
& =\int_{\Gamma \backslash G / K}\left(\Phi_{s, r}^{(d)}(g) \mid\left(\triangle+\bar{s}^{2}-\rho_{0}^{2}\right)^{r+1} \alpha(g)\right) \mathrm{d} g \\
& =\frac{\Gamma(q-1)}{\pi^{q}} \int_{\Gamma \backslash G} \sum_{\gamma \in \Gamma_{H} \backslash \Gamma}\left(\varphi_{s, r}^{(d)}(\gamma g) \mid\left(\triangle+\bar{s}^{2}-\rho_{0}^{2}\right)^{r+1} \alpha(\gamma g)\right) \mathrm{d} g \\
& =\frac{\Gamma(q-1)}{\pi^{q}} \int_{\Gamma_{H} \backslash G}\left(\varphi_{s, r}^{(d)}(g) \mid\left(\triangle+\bar{s}^{2}-\rho_{0}^{2}\right)^{r+1} \alpha(g)\right) \mathrm{d} g \\
& =\frac{\Gamma(q-1)}{\pi^{q}} \int_{0}^{+\infty}\left(\varphi_{s, r}^{(d)}\left(a_{t}\right) \mid \int_{\Gamma_{H} \backslash H}\left(\triangle+\bar{s}^{2}-\rho_{0}^{2}\right)^{r+1} \alpha\left(h a_{t}\right) \mathrm{d} h\right) \varrho(t) \mathrm{d} t .
\end{aligned}
$$

Since the support of the function $\alpha$ is compact modulo $\Gamma$ and since $\Gamma_{H} \backslash H$ is closed in $\Gamma \backslash G$, the integral $\alpha^{\Gamma_{H} \backslash H}(g)$ converges absolutely and defines a function $\alpha^{\Gamma_{H} \backslash H} \in C^{\infty}(G / K ; \tau)^{H}$. The map $\alpha \mapsto \alpha^{\Gamma_{H} \backslash H}$ is a $Z\left(\mathfrak{g}_{\mathbb{C}}\right)$-homomorphism from $\mathcal{A}_{\mathrm{C}}(\Gamma \backslash G / K)$ to $C^{\infty}(G / K ; \tau)^{H}$. By this remark and Proposition 14, we have this expression of $I_{r}(\alpha)$ in terms of the integral $\alpha^{\Gamma_{H} \backslash H}$ :

$$
I_{r}(\alpha)=\frac{\Gamma(q-1)}{\pi^{q}} \int_{0}^{+\infty}\left(\varphi_{s, r}^{(d)}\left(a_{t}\right) \mid f_{r+1}(t)\right) \varrho(t) \mathrm{d} t
$$

with $f_{r}(t)=\left(-\mathcal{D}_{t}+\bar{s}^{2}-\rho_{0}^{2}\right)^{r} \alpha^{\Gamma_{H} \backslash H}\left(a_{t}\right)$. Fix $0<\epsilon<R$. Then by Lemma 15, we have

$$
\begin{aligned}
& \int_{\epsilon}^{R}\left(\varphi_{s, r}^{(d)}\left(a_{t}\right) \mid f_{r+1}(t)\right) \varrho(t) \mathrm{d} t \\
& =\Re(R)-\Re(\epsilon)+\int_{\epsilon}^{R}\left(\left(-\mathcal{D}_{t}+s^{2}-\rho_{0}^{2}\right) \varphi_{s, r}^{(d)}\left(a_{t}\right) \mid f_{r}(t)\right) \varrho(t) \mathrm{d} t
\end{aligned}
$$

with

$$
\Re(t)=\varrho(t)\left\{-\left(\varphi_{s, r}^{(d)}\left(a_{t}\right) \mid \frac{\mathrm{d}}{\mathrm{d} t} f_{r}(t)\right)+\left(\frac{\mathrm{d}}{\mathrm{d} t} \varphi_{s, r}^{(d)}\left(a_{t}\right) \mid f_{r}(t)\right)\right\} .
$$

Let us compute the limit of $\Re(\epsilon)$ as $\epsilon \rightarrow+0$. As we noticed in the proof of Proposition 29 , we have the estimations $\varrho(t) \varphi_{s, r}^{(d)}\left(a_{t}\right)=O\left(\varrho(t) t^{-2 q+2 r+2}\right)=O\left(t^{2 r+1}\right)$ 
and $\varrho(t) \frac{d}{d t} \varphi_{s, r}^{(d)}\left(a_{t}\right)=O\left(t^{2 r}\right)$ for small $t>0$. Since $f_{r}(t)$ is continuous, we have $f_{r}(t)=O(1)$. Hence if $r>0$, then $\mathfrak{R}(t)=O(t)$ for small $t>0$, which implies $\lim _{\epsilon \rightarrow+0} \mathfrak{R}(\epsilon)=0$. When $r=0$, we compute the limit exactly the same way as in the proof of Proposition 23. Summing up, we obtain

$$
\lim _{\epsilon \rightarrow+0} \mathfrak{R}(\epsilon)=\frac{-4 \pi^{q}}{\Gamma(q-1)}\left(\Lambda^{q-d}\left(* \operatorname{vol}_{\mathfrak{p} \cap \mathfrak{h}}\right) \mid f_{0}(0)\right) \delta_{r, 0} .
$$

Let us compute the limit of $\mathfrak{R}(R)$ as $R \rightarrow+\infty$. By (5.2), the estimations $\varrho(t) \varphi_{r, s}^{(d)}\left(a_{t}\right)=O\left(e^{-\left(\operatorname{Re}(s)-\rho_{0}\right) t}\right)$ and $\varrho(t) \frac{\mathrm{d}}{\mathrm{d} t} \varphi_{r, s}^{(d)}\left(a_{t}\right)=O\left(e^{-\left(\operatorname{Re}(s)-\rho_{0}\right) t}\right)$ hold for large $t$. Since $f_{r}(t)$ is bounded on $t>0$, we have

$$
\lim _{R \rightarrow+\infty} \mathfrak{R}(R)=0 .
$$

By the formula $\left[s^{2}, \frac{1}{r !}\left(\frac{-1}{2 s} \frac{\mathrm{d}}{\mathrm{d} s}\right)^{r}\right]=\frac{1}{(r-1) !}\left(\frac{-1}{2 s} \frac{\mathrm{d}}{\mathrm{d} s}\right)^{r-1}$, we have

$$
\begin{aligned}
\left(-\mathcal{D}_{t}+s^{2}-\rho_{0}^{2}\right) \varphi_{s, r}^{(d)}\left(a_{t}\right) & =\frac{1}{r !}\left(\frac{-1}{2 s} \frac{\mathrm{d}}{\mathrm{d} s}\right)^{r}\left(-\mathcal{D}_{t}+s^{2}-\rho_{0}^{2}\right) \varphi_{s}^{(d)}\left(a_{t}\right)+\varphi_{s, r-1}^{(d)}\left(a_{t}\right) \\
& =\varphi_{s, r-1}^{(d)}\left(a_{t}\right)
\end{aligned}
$$

using $\left(-\mathcal{D}_{t}+s^{2}-\rho_{0}^{2}\right) \varphi_{s}^{(d)}\left(a_{t}\right)=0(\forall t>0)$. Hence taking the limit as $\epsilon \rightarrow+0$, $R \rightarrow+\infty$ of (6.4) and using (6.5), (6.6) and (6.7), from (6.3), we obtain

$$
\begin{aligned}
& I_{r}(\alpha) \\
& =4\left(\Lambda^{q-d}\left(* \operatorname{vol}_{\mathfrak{p} \cap \mathfrak{h}}\right) \mid f_{0}(0)\right) \delta_{r, 0}+\frac{\Gamma(q-1)}{\pi^{q}} \int_{0}^{+\infty}\left(\left(-\mathcal{D}_{t}+s^{2}-\rho_{0}^{2}\right) \varphi_{s, r}^{(d)}\left(a_{t}\right) \mid f_{r}(t)\right) \varrho(t) \mathrm{d} t \\
& =4\left(\Lambda^{q-d}\left(* \operatorname{vol}_{\mathfrak{p} \cap \mathfrak{h}}\right) \mid \alpha^{\Gamma_{H} \backslash H}(e)\right) \delta_{r, 0}+\frac{\Gamma(q-1)}{\pi^{q}} \int_{0}^{+\infty}\left(\varphi_{s, r-1}^{(d)}\left(a_{t}\right) \mid f_{r}(t)\right) \varrho(t) \mathrm{d} t \\
& =\left\langle 4 \Lambda^{q-d}\left(* \operatorname{vol}_{\mathfrak{p} \cap \mathfrak{h}}\right), * \bar{\alpha}\right\rangle \delta_{r, 0}+I_{r-1}(\alpha) .
\end{aligned}
$$

Note the last equality is by Lemma 30 .

6.3. Spectral expansions of Poincaré series. In order to obtain a meromorphic continuation of the function $s \mapsto \Phi_{s, 0}^{(d)}$ beyond the convergence region $\operatorname{Re}(s)>\rho_{0}$, we want to use the $L^{2}$-theory, i.e., the spectral decomposition of the Laplace-Beltrami operator acting on the Hilbert space of square integrable $(d, d)$-forms. Unfortunately, the form $\Phi_{s, 0}^{(d)}$ is not square-integrable, even when $\Gamma \backslash G$ is compact. This difficulty is circumvented by considering $\Phi_{s, r}^{(d)}$ with a large $r(c f .[8])$. 
Proposition 32. Let $r \geqslant q-1$. Suppose one of the conditions (a) and (b) is satisfied:

(a) $\Gamma \backslash G$ is compact, and $\operatorname{Re}(s)>\rho_{0}$.

(b) $\Gamma \backslash G$ is non compact, and $\operatorname{Re}(s)>\rho_{0}\left(3-2 p^{-1}\right)$.

Then the measurable $(d, d)$-form $\Phi_{s, r}^{(d)}$ on $\Gamma \backslash G / K$ is $L^{2+\epsilon}$ for some $\epsilon>0$.

Proof. From Proposition 25 (3), the term $\sum_{\alpha=0}^{q-2} \mathrm{c}_{\alpha}(s) z^{-q+\alpha+1}$ in the formula (5.25) is annihilated by $\left(\frac{-1}{2 s} \frac{d}{d s}\right)^{r}$ if $r \geqslant q-1$. Hence

$$
\varphi_{s, r}^{(d)}\left(a_{t}\right)=\mathrm{P}_{r}\left(s, \tanh ^{2} t\right)+\log \left(\tanh ^{2} t\right) \mathrm{Q}_{r}\left(s, \tanh ^{2} t\right)
$$

for small $t>0$ with $\mathrm{P}_{r}(s, z)=\frac{1}{r !}\left(\frac{-1}{2 s} \frac{d}{d s}\right)^{r} \mathrm{P}(s, z)$ and $\mathrm{Q}_{r}(s, z)=\frac{1}{r !}\left(\frac{-1}{2 s} \frac{d}{d s}\right)^{r} \mathrm{Q}(s, z)$. For large $t>0$, the estimation $\varphi_{s, r}^{(d)}\left(a_{t}\right)=O\left(e^{-\left(\operatorname{Re}(s)+\rho_{0}\right) t}\right)$ holds as we noticed in Proposition 29. Using these estimation of $\varphi_{s, r}^{(d)}\left(a_{t}\right)$, we can argue exactly the same way as $[26$, Section 5] to have the conclusion.

Remark: Since $\operatorname{vol}(\Gamma \backslash G)<+\infty$, Hölder's inequality yields the inclusion

$$
L^{2+\epsilon}(\Gamma \backslash G) \subset L^{2}(\Gamma \backslash G) .
$$

Let $\mathcal{A}_{(2)}^{d, d}(\Gamma \backslash G / K)$ be the completion of the space $\mathcal{A}_{\mathrm{c}}^{d, d}(\Gamma \backslash G / K)$ by the inner product

$$
\langle\alpha \mid \beta\rangle=\int_{\Gamma \backslash G / K} \alpha \wedge * \bar{\beta} .
$$

From now on, we further assume that $\Gamma$ is a uniform lattice, i.e., the manifold $\Gamma \backslash G / K$ is compact. Then the Hodge Laplacian $\triangle$ with the domain $\mathcal{A}^{d, d}(\Gamma \backslash G / K)$ is essentially self-adjoint operator on the Hilbert space $\mathcal{A}_{(2)}^{d, d}(\Gamma \backslash G / K)$. The domain of $\bar{\triangle}$, the minimal closed extension of $\triangle$, consists of all $\alpha \in \mathcal{A}_{(2)}^{d, d}(\Gamma \backslash G / K)$ such that the distribution $\triangle \alpha$ belongs to $\mathcal{A}_{(2)}^{d, d}(\Gamma \backslash G / K)$. There exists an orthonormal basis $\left\{\alpha_{n}\right\}_{n \in \mathbb{N}}$ of $\mathcal{A}_{(2)}^{d, d}(\Gamma \backslash G / K)$ consisting of eigenvectors of $\bar{\triangle}$; let $\left\{\lambda_{n}\right\}$ be the corresponding system of eigenvalues: $\bar{\triangle} \alpha_{n}=\lambda_{n} \alpha_{n}$. Note $\alpha_{n}$ 's are $C^{\infty}$-forms and $\lambda_{n}$ 's are non-negative real numbers because the differential operator $\triangle$ is positive, formally self-adjoint and elliptic. 
The Secondary Spherical Functions and Automorphic Green ... 1017

Theorem 33. Let $r \geqslant q-1$ and $\operatorname{Re}(s)>\rho_{0}$. Then

$$
\Phi_{s, r}^{(d)}=\sum_{n=0}^{\infty} \frac{4\left\langle\Lambda^{q-d} \delta_{C}, * \bar{\alpha}_{n}\right\rangle}{\left(\lambda_{n}+s^{2}-\rho_{0}^{2}\right)^{r+1}} \alpha_{n}
$$

is the spectral expansion of $\Phi_{s, r}^{(d)} \in \mathcal{A}_{(2)}^{d, d}(\Gamma \backslash G / K)$.

Proof. Since $\Gamma \backslash G / K$ is compact by assumption and since $\Phi_{s, r}^{(d)} \in \mathcal{A}_{(2)}(\Gamma \backslash G / K)$ by Proposition 32, we have $\Phi_{s, r}^{(d)}=\sum_{n}\left\langle\Phi_{s, r}^{(d)} \mid \alpha_{n}\right\rangle \alpha_{n}$ in a weak sense. By Theorem 31, we can evaluate the coefficient of $\alpha_{n}$ concretely :

$$
\left\langle\Phi_{s, r}^{(d)} \mid \alpha_{n}\right\rangle=\frac{4\left\langle\Lambda^{q-d} \delta_{C}, * \bar{\alpha}_{n}\right\rangle}{\left(\lambda_{n}+s^{2}-\rho_{0}^{2}\right)^{r+1}} .
$$

The spectral expansion immediately yields a meromorphic continuation of $s \mapsto$ $\Phi_{s, r}^{(d)}$ if $r \geqslant q-1$. After a bit more argument, we can remove the restriction on $r$.

Theorem 34. Let $r \in \mathbb{N}$ and $\beta \in \mathcal{A}(\Gamma \backslash G / K)$. The function $s \mapsto\left\langle\Phi_{s, r}^{(d)} \mid \beta\right\rangle$ has a meromorphic continuation to the whole complex plane $\mathbb{C}$. A point $s_{0} \in \mathbb{C}$ with $\operatorname{Re}\left(s_{0}\right) \geqslant 0$ is a pole of $\left\langle\Phi_{s, r}^{(d)} \mid \beta\right\rangle$ if and only if there exists an index $n \in \mathbb{N}$ such that $\left\langle\Lambda^{q-d} \delta_{C}, * \bar{\alpha}_{n}\right\rangle \neq 0,\left\langle\alpha_{n} \mid \beta\right\rangle \neq 0$ and $s_{0}^{2}-\rho_{0}^{2}=-\lambda_{n}$. The function

$$
\left\langle\Phi_{s, r}^{(d)} \mid \beta\right\rangle+\sum_{\substack{n \in \mathbb{N} \\ \lambda_{n}=\rho_{0}^{2}-s_{0}^{2}}} \frac{-4\left\langle\Lambda^{q-d} \delta_{C}, * \bar{\alpha}_{n}\right\rangle}{\left(\lambda_{n}+s^{2}-\rho_{0}^{2}\right)^{r+1}}\left\langle\alpha_{n} \mid \beta\right\rangle
$$

is holomorphic at $s=s_{0}$. We have the functional equation $\Phi_{s, r}^{(d)}=\Phi_{-s, r}^{(d)}$.

Proof. Suppose first $r \geqslant q-1$ and fix a point $s_{0} \in \mathbb{C}$ such that $\operatorname{Re}\left(s_{0}\right)>\rho_{0}$. Given a relatively compact open set $U$ disjoint from the discrete set $S=\left\{s \in \mathbb{C} \mid s^{2}=\right.$ $\left.\rho_{0}^{2}-\lambda_{n}(\exists n \in \mathbb{N})\right\}$, there exists a constant $C_{U}>0$ such that $\left|\lambda_{n}-\rho_{0}^{2}+s_{0}^{2}\right| \leqslant$ $C_{U}\left|\lambda_{n}-\rho_{0}^{2}+s^{2}\right|(\forall n \in \mathbb{N}, \forall s \in U)$. Using this and the Parseval equality, we obtain

$$
\begin{aligned}
\sum_{n \in \mathbb{N}} \sup _{s \in U} \frac{\left|4\left\langle\Lambda^{q-d} \delta_{C}, * \bar{\alpha}_{n}\right\rangle\right|}{\left|\lambda_{n}+s^{2}-\rho_{0}^{2}\right| r^{r+1}}\left|\left\langle\alpha_{n} \mid \beta\right\rangle\right| & \leqslant C_{U}^{r+1}\left\{\sum_{n \in \mathbb{N}}\left|\left\langle\alpha_{n} \mid \beta\right\rangle\right|^{2}\right\}^{1 / 2} \cdot\left\{\sum_{n \in \mathbb{N}} \frac{\left|4\left\langle\Lambda^{q-d} \delta_{C}, * \bar{\alpha}_{n}\right\rangle\right|^{2}}{\left|\lambda_{n}+s_{0}^{2}-\rho_{0}^{2}\right|^{2(r+1)}}\right\}^{1 / 2} \\
& =C_{U}^{r+1}\|\beta\|\left\|\Phi_{s_{0}, r}^{(d)}\right\|<+\infty
\end{aligned}
$$


This shows that the series

$$
\left\langle\Phi_{s, r}^{(d)} \mid \beta\right\rangle=\sum_{n} \frac{4\left\langle\Lambda^{q-d} \delta_{C}, * \bar{\alpha}\right\rangle}{\left(\lambda_{n}-\rho_{0}^{2}+s^{2}\right)^{r+1}}\left\langle\alpha_{n} \mid \beta\right\rangle
$$

converges absolutely and uniformly on arbitrary compact set disjoint from $S$, providing a meromorphic analytic continuation of $\left\langle\Phi_{s, r}^{(d)} \mid \beta\right\rangle$ to the whole $\mathbb{C}$. The remaining assertions are also obvious from this formula.

We use a downward-induction to establish the theorem for $r$, assuming it holds for $r+1$. Fix $\beta \in \mathcal{A}(\Gamma \backslash G / K)$. Then there exists a meromorphic function $F_{r+1}(s)$ on $\mathbb{C}$ such that $F_{r+1}(s)=\left\langle\Phi_{s, r+1}^{(d)} \mid \beta\right\rangle\left(\operatorname{Re}(s)>\rho_{0}\right)$ and $F_{r+1}(s)+$ $\sum_{\substack{n \in \mathbb{N} \\ \lambda_{n}=\rho_{0}^{2}-s_{0}^{2}}} \frac{4\left\langle\Lambda^{q-d} \delta_{C}, * \bar{\alpha}_{n}\right\rangle}{\left(\lambda_{n}+s^{2}-\rho_{0}^{2}\right)^{r+2}}\left\langle\alpha_{n} \mid \beta\right\rangle$ is holomorphic at $s=s_{0}$ for each $s_{0} \in \mathbb{C}$. This implies the residue of $s F_{r+1}(s)$ at its arbitrary pole is zero, which guarantees that the integral

$$
F_{r}(s)=-2(r+1) \int_{L_{s}} \zeta F_{r+1}(\zeta) \mathrm{d} \zeta+\left\langle\Phi_{s_{0}, r}^{(d)} \mid \beta\right\rangle
$$

is independent of the choice of a path $L_{s}$ connecting $s_{0}$ and $s$ in $\mathbb{C}-S$. Since $\frac{-1}{2(r+1) s} \frac{\mathrm{d}}{\mathrm{d} s}\left\langle\Phi_{s, r}^{(d)} \mid \beta\right\rangle=F_{r+1}(s)$ on $\operatorname{Re}(s)>\rho_{0}$, the function $F_{r}(s)$ defined by (6.8) establishes a meromorphic analytic continuation of $\left\langle\Phi_{s, r}^{(d)} \mid \beta\right\rangle\left(\operatorname{Re}(s)>\rho_{0}\right)$ to the whole $\mathbb{C}$. All the assertions except the functional equation $F_{r}(s)=F_{r}(-s)$ are obvious from (6.8). It remains to prove the functional equation. By inductionassumption, $F_{r+1}(s)=F_{r+1}(-s)$ holds. Therefore,

$$
\frac{\mathrm{d}}{\mathrm{d} s}\left(F_{r}(s)-F_{r}(-s)\right)=F_{r}^{\prime}(s)+F_{r}^{\prime}(-s)=-2(r+1) s\left\{F_{r+1}(s)-F_{r+1}(-s)\right\}=0 .
$$

This implies the difference $F_{r}(s)-F_{r}(-s)$ is a constant, which should be zero since $F_{r}(s)-F_{r}(-s)$ is an odd function of $s$. Hence $F_{r}(s)=F_{r}(-s)$ as desired.

\section{Automorphic Poincaré dual forms and Green Currents}

Set $G_{H}^{\Gamma}(s)=\Phi_{s, 0}^{(q-1)}$ and $\Psi_{H}^{\Gamma}(s)=\Phi_{s, 0}^{(q)}$.

Theorem 35. The equations

$$
\begin{aligned}
\left(\triangle+s^{2}-\rho_{0}^{2}\right) G_{H}^{\Gamma}(s) & =4 \Lambda \delta_{C_{H}^{\Gamma},} \\
\left(\triangle+s^{2}-\rho_{0}^{2}\right) \Psi_{H}^{\Gamma}(s) & =4 \delta_{C_{H}^{\Gamma},} \\
\mathrm{d}_{\mathrm{c}} \mathrm{d} G_{H}^{\Gamma}(s)+\left(s^{2}-\rho_{0}^{2}\right) \Psi_{H}^{\Gamma}(s) & =4 \delta_{C_{H}^{\Gamma}}
\end{aligned}
$$


hold for $s$ outside the poles of $G_{H}^{\Gamma}(s)$ and $\Psi_{H}^{\Gamma}(s)$.

Proof. Since the first two equation is already proved in Theorem 31, it suffices to show the third equation (7.1). Let $\alpha \in \mathcal{A}_{\mathrm{c}}(\Gamma \backslash G / K)$. Then, in the same way as the computation (6.2),

$$
\begin{aligned}
\left\langle\frac{1}{4} \mathrm{~d}_{\mathrm{c}} \mathrm{d} G_{H}^{\Gamma}(s), * \bar{\alpha}\right\rangle & =\frac{1}{2 \sqrt{-1}}\left\langle\partial \bar{\partial} G_{H}^{\Gamma}(s), * \bar{\alpha}\right\rangle \\
& =\frac{\sqrt{-1}}{2}\left\langle G_{H}^{\Gamma}(s), \bar{\partial} \partial * \bar{\alpha}\right\rangle \\
& =\frac{-\sqrt{-1}}{2}\left\langle G_{H}^{\Gamma}(s), * \overline{\partial^{*}} \partial^{*} \alpha\right\rangle \\
& =\frac{-\sqrt{-1} \Gamma(q-1)}{2 \pi^{q}} \int_{0}^{+\infty} \varrho(t)\left(\phi_{s}\left(a_{t}\right) \mid\left(\bar{\partial}^{*} \partial^{*} \alpha\right)^{\Gamma_{H} \backslash H}\left(a_{t}\right)\right) \mathrm{d} t \\
& =\frac{-\sqrt{-1} \Gamma(q-1)}{2 \pi^{q}} \int_{0}^{+\infty} \varrho(t)\left(\phi_{s}\left(a_{t}\right) \mid\left(\bar{\partial}^{*} \partial^{*}\left(\alpha^{\Gamma_{H} \backslash H}\right)\right)\left(a_{t}\right)\right) \mathrm{d} t \\
& =\frac{-\sqrt{-1} \Gamma(q-1)}{2 \pi^{q}} \int_{0}^{+\infty} \varrho(t)\left(\phi_{s}\left(a_{t}\right) \mid \mathcal{E}_{t}^{*}\left\{\alpha^{\Gamma_{H} \backslash H}\left(a_{t}\right)\right\}\right) \mathrm{d} t .
\end{aligned}
$$

Here we use Lemma 16 to obtain the last equality. Fix $0<\epsilon<R$. Then by Lemma 17, using Theorem 26 and Proposition 16, we have

$\int_{\epsilon}^{R}\left(\phi_{s}\left(a_{t}\right) \mid \mathcal{E}_{t}^{*} f(t)\right) \varrho(t) \mathrm{d} t=-\mathfrak{S}(R)+\mathfrak{S}(\epsilon)+\frac{-\sqrt{-1}\left(s^{2}-\rho_{0}^{2}\right)}{2} \int_{\epsilon}^{R}\left(\psi_{s}\left(a_{t}\right) \mid f(t)\right) \varrho(t) \mathrm{d} t$,

with $f(t)=\alpha^{\Gamma_{H} \backslash H}\left(a_{t}\right)(\forall t>0)$ and

$$
\begin{aligned}
\mathfrak{S}(t)= & \frac{\varrho(t)}{4}\left\{\left(e\left(\omega_{0} \wedge \bar{\omega}_{0}\right) \frac{\mathrm{d}}{\mathrm{d} t} \phi_{s}\left(a_{t}\right) \mid f(t)\right)-\left(e\left(\omega_{0} \wedge \bar{\omega}_{0}\right) \phi_{s}\left(a_{t}\right) \mid f^{\prime}(t)\right)\right. \\
& +\tanh t\left(\left(2 \mathcal{A}-(2 p-1) e\left(\omega_{0} \wedge \bar{\omega}_{0}\right)\right) \phi_{s}\left(a_{t}\right) \mid f(t)\right) \\
& \left.+\operatorname{coth} t\left(\left(2 \mathcal{B}-(2 q-1) e\left(\omega_{0} \wedge \bar{\omega}_{0}\right)\right) \phi_{s}\left(a_{t}\right) \mid f(t)\right)\right\} .
\end{aligned}
$$

We have

$$
\begin{aligned}
\mathfrak{S}(t) & \\
= & \frac{\varrho(t)}{4}\left\{\left(e\left(\omega_{0} \wedge \bar{\omega}_{0}\right) \frac{\mathrm{d}}{\mathrm{d} t} \phi_{s}\left(a_{t}\right) \mid f(t)\right)\right. \\
& \left.+\operatorname{coth} t\left(\left(2 \mathcal{B}-(2 q-1) e\left(\omega_{0} \wedge \bar{\omega}_{0}\right)\right) \phi_{s}\left(a_{t}\right) \mid f(t)\right)\right\}+O(t \log t) \\
= & \frac{1}{4} \frac{2 \pi^{q}}{\Gamma(q)}\left(\left\{-2(q-1) e\left(\omega_{0} \wedge \bar{\omega}_{0}\right) \Lambda\left(* \operatorname{vol}_{\mathfrak{p} \cap \mathfrak{h}}\right)\right.\right. \\
& \left.\left.+\left(2 \mathcal{B}-(2 q-1) e\left(\omega_{0} \wedge \bar{\omega}_{0}\right)\right) \Lambda\left(* \operatorname{vol}_{\mathfrak{p} \cap \mathfrak{h}}\right)\right\} \mid f(0)\right)+O(t \log t) \\
= & \frac{-\pi^{q}}{\Gamma(q-1)}\left(e\left(\omega_{0} \wedge \bar{\omega}_{0}\right) \Lambda\left(* \operatorname{vol}_{\mathfrak{p} \cap \mathfrak{h}}\right) \mid f(0)\right)+O(t \log t),
\end{aligned}
$$


since $\left(2 \mathcal{B}-(2 q-1) e\left(\omega_{0} \wedge \bar{\omega}_{0}\right)\right) \Lambda\left(* \operatorname{vol}_{\mathfrak{p} \cap \mathfrak{h}}\right)=0$ by Lemma 27 and Lemma 7 . Therefore, we obtain

$$
\begin{aligned}
\lim _{\epsilon \rightarrow+0} \mathfrak{S}(\epsilon) & =\frac{-\pi^{q}}{\Gamma(q-1)}\left(e\left(\omega_{0} \wedge \bar{\omega}_{0}\right) \Lambda\left(* \operatorname{vol}_{\mathfrak{p} \cap \mathfrak{h}}\right) \mid f(0)\right) \\
& =\frac{-\pi^{q}}{\Gamma(q-1)}\left(e\left(\omega_{0} \wedge \bar{\omega}_{0}\right) \frac{1}{(q-1) !} \gamma_{p p}^{q-1} \mid f(0)\right) \\
& =\frac{-\pi^{q}}{\Gamma(q-1)} \frac{2}{\sqrt{-1}} \frac{1}{q !}\left(\gamma_{p p}^{q} \mid f(0)\right) \\
& =\frac{-2 \pi^{q}}{\sqrt{-1} \Gamma(q-1)}\left(* \operatorname{vol}_{\mathfrak{p} \cap \mathfrak{h}} \mid f(0)\right)
\end{aligned}
$$

by the first formula in Lemma 28 and Lemma 7. Since $f(t)$ is bounded,

$$
\lim _{R \rightarrow+\infty} \mathfrak{S}(R)=0
$$

is proved similarly to the corresponding part in the proof of Proposition 31. From (7.2), (7.3), (7.5) and (7.6),

$$
\begin{aligned}
\left\langle\frac{1}{4} \mathrm{~d}_{\mathrm{c}} \mathrm{d} G_{H}^{\Gamma}(s), * \bar{\alpha}\right\rangle & =\left(* \operatorname{vol}_{\mathfrak{p} \cap \mathfrak{h}} \mid f(0)\right)-\frac{s^{2}-\rho_{0}^{2}}{4} \frac{\Gamma(q-1)}{\pi^{q}} \int_{0}^{+\infty}\left(\psi_{s}\left(a_{t}\right) \mid f(t)\right) \varrho(t) \mathrm{d} t \\
& =\left\langle\delta_{C}, * \bar{\alpha}\right\rangle-\frac{s^{2}-\rho_{0}^{2}}{4}\left\langle\Psi_{H}^{\Gamma}(s), * \bar{\alpha}\right\rangle .
\end{aligned}
$$

Here the last equality follows from Lemma 30 and by a similar computation we did to prove (6.2). This completes the proof.

Since $G_{H}^{\Gamma}(s)$ and $\Psi_{H}^{\Gamma}(s)$ are meromorphic on $\mathbb{C}$ with at most simple poles at $s=\rho_{0}$, we can consider the constant term and the residue of their Laurent expansion:

$$
\mathcal{G}_{H}^{\Gamma}=\frac{1}{4} \mathrm{CT}_{s=\rho_{0}} G_{H}^{\Gamma}(s), \quad \Psi_{H}^{\Gamma}=\frac{\rho_{0}}{2} \operatorname{Res}_{s=\rho_{0}} \Psi_{H}^{\Gamma}(s) .
$$

Theorem 36. We have

$$
\triangle \mathcal{G}_{H}^{\Gamma}=\Lambda \delta_{C_{H}^{\Gamma}}, \quad \triangle \Psi_{H}^{\Gamma}=0, \quad \mathrm{~d}_{\mathrm{c}} \mathrm{d} \mathcal{G}_{H}^{\Gamma}+\Psi_{H}^{\Gamma}=\delta_{C_{H}^{\Gamma}} .
$$

Proof. The first equation is obtained by comparing the constant terms of the Laurent expansion at $s=\rho_{0}$ in both sides of the first equation of Theorem 35 . The last equation is justified by the equation (7.1) in the same way. The second equation is proved by taking the residue at $s=\rho_{0}$ of the second equation of Theorem 35.

Remark 1: By the Hodge theory for compact Kähler manifolds, the fundamental class of the cycle $C_{H}^{\Gamma}$ has a unique harmonic representative in $\mathcal{A}^{q, q}(\Gamma \backslash G / K)$ called the Poincaré dual form of $C_{H}^{\Gamma}$. Our result tells an explicit way how to construct 
that harmonic form. Indeed, the second equation in Theorem 36 shows the $(q, q)$ form $\Psi_{H}^{\Gamma}$ is harmonic and the third one means $\Psi_{H}^{\Gamma}$ is cohomologus to the current $\delta_{C_{H}^{\Gamma}}$. Therefore, $\Psi_{H}^{\Gamma}$ meets the requirements of the Poincaré dual form.

Remark 2: Theorem 36 also tells that $(q-1, q-1)$-current $\mathcal{G}_{H}^{\Gamma}$ is a Green current for the cycle $C_{H}^{\Gamma}$ in the sense of Gillet-Soulé [5]. Though there are many Green currents for $C_{H}^{\Gamma}$, our construction fixes a choice, whose dependence on $\Gamma$ is tractable. Another advantage of our choice $\mathcal{G}_{H}^{\Gamma}$ is that the form $\Psi_{H}^{\Gamma}$ is harmonic.

\section{Some global COnsequences on CyCle Geometry}

Along the $K$-module decomposition (3.7), the current $\Psi_{H}^{\Gamma}(s)$ is decomposed as

$$
\Psi_{H}^{\Gamma}(s)=\sum_{\kappa=0}^{q} \Psi_{H, \kappa}^{\Gamma}(s), \quad \Psi_{H, \kappa}^{\Gamma}(s) \in C^{\infty}\left((G-\Gamma H K) / K ; V_{\kappa}^{(d)}\right)^{\Gamma} .
$$

Each component function $\Psi_{H, \kappa}^{\Gamma}(s)$ is also meromorphic in $s \in \mathbb{C}$ and the Poincaré dual form $\Psi_{H}^{\Gamma}$ is a sum of forms $\Psi_{H, \kappa}^{\Gamma}=\frac{\rho_{0}}{2} \operatorname{Res}_{s=\rho_{0}} \Psi_{H, \kappa}^{\Gamma}(s)(0 \leqslant \kappa \leqslant q)$, each of which is also harmonic. Moreover $\Psi_{H, q}^{\Gamma}$ is primitive, i.e., $\Lambda \Psi_{H, q}^{\Gamma}=0$. The aim of this section is to study these forms $\Psi_{H, \kappa}^{\Gamma}$ by using the knowledge on the $\left(\mathfrak{g}_{\mathbb{C}}, K\right)$-module they generate in the space of $L^{2}$-automorphic forms.

The form $\Psi_{H, 0}^{\Gamma}$ is fairly easy to deal with.

Proposition 37. We have

$$
\Psi_{H, 0}^{\Gamma}=\frac{\operatorname{vol}\left(\Gamma_{H} \backslash H / K_{H}\right)}{\operatorname{vol}(\Gamma \backslash G / K)} \theta_{0}^{(q)} .
$$

In particular, $\Psi_{H, 0}^{\Gamma} \neq 0$.

Proof. Since $\theta_{0}^{(q)}$ is a $K$-fixed tensor, it is obvious from the construction that $\Psi_{H, 0}^{\Gamma}(s ; g)=\Phi_{s}^{0}(g) \theta_{0}^{(q)}$, where

$$
\Phi_{s}^{0}(g)=\frac{\Gamma(q-1)}{\pi^{q}} \sum_{\gamma \in \Gamma_{H} \backslash \Gamma} \varphi_{s}^{0}(\gamma g), \quad \operatorname{Re}(s)>\rho_{0}
$$

with $\varphi_{s}^{0} \in C^{\infty}((G-H K) / K)$, a scalar valued function, such that $\varphi_{s}^{0}\left(a_{t}\right)=$ $F_{0}(s ; t)(\forall t>0)$. Set $f(g)=\operatorname{Res}_{s=\rho_{0}} \Phi_{s}^{0}(g)$. Then $f \in L^{2}(\Gamma \backslash G / K)$ and $\Psi_{H, 0}^{\Gamma}=$ $\frac{\rho_{0}}{2} f(g) \theta_{0}^{(q)}$. From $\triangle \Psi_{H, 0}^{\Gamma}=0$, the function $f(g)$ should be a harmonic function 
of the compact Riemanian manifold $\Gamma \backslash G / K$. Hence $f(g)$ has to be a constant, say $C$. To determine this constant $C$, we compute the integral

$$
\begin{aligned}
& \frac{1}{\operatorname{vol}\left(\Gamma_{H} \backslash H\right)} \int_{\Gamma \backslash G} \Phi_{s}^{0}(g) \mathrm{d} g \\
= & \frac{1}{\operatorname{vol}\left(\Gamma_{H} \backslash H\right)} \frac{\Gamma(q-1)}{\pi^{q}} \int_{\Gamma_{H} \backslash G} \varphi_{s}^{0}(g) \mathrm{d} g \\
= & \frac{\Gamma(q-1)}{\pi^{q}} \int_{0}^{+\infty} F_{0}(s ; t) \varrho(t) \mathrm{d} t \\
= & \frac{2}{q-1} \frac{\Gamma\left(\frac{s+\rho_{0}}{2}\right) \Gamma\left(\frac{s-\rho_{0}}{2}+q\right)}{\Gamma(s+1) \Gamma(q-1)} \int_{0}^{+\infty}(\sinh t)^{2 q-1}(\cosh t)^{-\left(s+\rho_{0}-2 p+1\right)} \\
& \times{ }_{2} F_{1}\left(\frac{s+\rho_{0}}{2}, \frac{s-\rho_{0}}{2}+q ; s+1 ; \frac{1}{\cosh ^{2} t}\right) \mathrm{d} t \\
= & \frac{2}{q-1} \frac{\Gamma\left(\frac{s+\rho_{0}}{2}\right) \Gamma\left(\frac{s-\rho_{0}}{2}+q\right)}{\Gamma(s+1) \Gamma(q-1)} \frac{1}{2} \int_{0}^{1}(1-z)^{q-1} z^{\frac{s-\rho_{0}-2}{2}}{ }_{2} F_{1}\left(\frac{s+\rho_{0}}{2}, \frac{s-\rho_{0}}{2}+q ; s+1 ; z\right) \mathrm{d} z \\
= & \frac{1}{q-1} \frac{\Gamma\left(\frac{s+\rho_{0}}{2}\right) \Gamma\left(\frac{s-\rho_{0}}{2}+q\right)}{\Gamma(s+1) \Gamma(q-1)} \frac{\Gamma(s+1) \Gamma\left(\frac{s-\rho_{0}}{2}\right) \Gamma(q)}{\Gamma\left(\frac{s-\rho_{0}}{2}+q\right) \Gamma\left(\frac{s-\rho_{0}}{2}+1\right) \Gamma\left(\frac{s+\rho_{0}}{2}+1\right)} \\
= & \frac{\Gamma\left(\frac{s-\rho_{0}}{2}\right) \Gamma\left(\frac{s+\rho_{0}}{2}\right)}{\Gamma\left(\frac{s-\rho_{0}}{2}+1\right) \Gamma\left(\frac{s+\rho_{0}}{2}+1\right)} .
\end{aligned}
$$

Here we use the integration formula (2.3) to have the second equality, make a change of variable $z=\frac{1}{\cosh ^{2} t}$ to obtain the fourth equality and use the formula $[9$, $7-512,3($ p.806)] to prove the fifth equality when $\operatorname{Re}(s)$ is sufficiently large. Taking the residue at $s=\rho_{0}$, we have

$$
\frac{\operatorname{vol}(\Gamma \backslash G)}{\operatorname{vol}\left(\Gamma_{H} \backslash H\right)} C=\operatorname{Res}_{s=\rho_{0}} \frac{\Gamma\left(\frac{s-\rho_{0}}{2}\right) \Gamma\left(\frac{s+\rho_{0}}{2}\right)}{\Gamma\left(\frac{s-\rho_{0}}{2}+1\right) \Gamma\left(\frac{s+\rho_{0}}{2}+1\right)}=2 \frac{\Gamma\left(\rho_{0}\right)}{\Gamma\left(\rho_{0}+1\right)} .
$$

Hence $\Psi_{H, 0}^{\Gamma}=\frac{\rho_{0}}{2} C \theta_{0}^{(q)}=\frac{\operatorname{vol}\left(\Gamma_{H} \backslash H\right)}{\operatorname{vol}(\Gamma \backslash G)} \theta_{0}^{(q)}$ as desired.

To investigate the other components $\Psi_{H, \kappa}^{\Gamma}(1 \leqslant \kappa \leqslant q)$, we recall basic facts about cohomological unitary representations (Vogan-Zuckerman [34], Wong [36], Vogan [35]).

Let $T\left(\cong \mathrm{U}(1)^{p+q}\right)$ be the compact Cartan subgroup of $G$ formed by all the

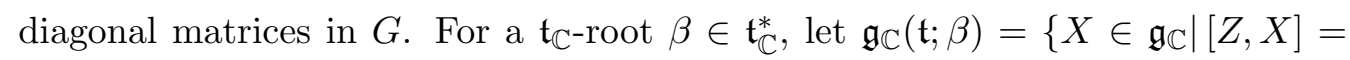
$\beta(Z) X(\forall Z \in \mathfrak{t})\}$ be the corresponding root space in $\mathfrak{g}_{\mathbb{C}}$. Note a $\mathfrak{t}$-root $\beta$ is real valued on $\sqrt{-1} \mathfrak{t}$. For $H_{0} \in \sqrt{-1} \mathfrak{t}$, let $\mathfrak{q}\left(H_{0}\right)$ be the $\theta$-stable parabolic subalgebra of $\mathfrak{g}$ with Levi part $\mathfrak{l}\left(H_{0}\right)=\left\{X \in \mathfrak{g}_{\mathbb{C}} \mid\left[H_{0}, X\right]=0\right\}$, and whose radical $\mathfrak{u}\left(H_{0}\right)$ is the sum of those root spaces $\mathfrak{g}_{\mathbb{C}}(\mathfrak{t} ; \beta)$ such that $\beta\left(H_{0}\right)>0$. The $\theta$-stable parabolic 
subalgebra $\mathfrak{q}=\mathfrak{q}\left(H_{0}\right)$ determines a unitarizable irreducible $\left(\mathfrak{g}_{\mathbb{C}}, K\right)$-module $A_{\mathfrak{q}}$ with non trivial cohomology $\mathrm{H}^{*}\left(\mathfrak{g}_{\mathbb{C}}, K ; A_{\mathfrak{q}}\right) \neq 0 ; A_{\mathfrak{q}}$ is characterized as a unique irreducible unitarizable $\left(\mathfrak{g}_{\mathbb{C}}, K\right)$-module with the two properties: (1) $A_{\mathfrak{q}}$ contains the $K$-type of highest weight $\lambda(\mathfrak{q})=2 \rho\left(\mathfrak{u}\left(H_{0}\right) \cap \mathfrak{p}_{\mathbb{C}}\right)$, the sum of those $T$-roots $\beta$ occurring in $\mathfrak{u}\left(H_{0}\right) \cap \mathfrak{p}_{\mathbb{C}} ;(2) \Omega_{\mathfrak{g}}$ acts by 0 ([34, Proposition 6.1$\left.]\right)$.

About the intermediate forms $\Psi_{H, \kappa}^{\Gamma}(1 \leqslant \kappa \leqslant q-1)$, we have the vanishing theorem.

Proposition 38. For $0<\kappa<q$, we have $\Psi_{H, \kappa}^{\Gamma}=0$.

Proof. On the contrary, suppose that $\Psi_{H, \kappa}^{\Gamma} \neq 0$ with $0<\kappa<q$. Let $\mathcal{V}$ be the closed $G$-submodule of $L^{2}(\Gamma \backslash G)$ generated by the coefficient functions $\Psi_{H, \kappa}^{\Gamma}(v ; g)=\left(\Psi_{H, \kappa}^{\Gamma}(g) \mid v\right)$ with $v \in V_{\kappa}^{(q)}$; by assumption $\mathcal{V} \neq\{0\}$. Since $\Gamma \backslash G$ is compact, the unitary representation $L^{2}(\Gamma \backslash G)$ is discretely decomposable, a fortiori its closed submodule $\mathcal{V}$ is. In particular, $\mathcal{V}$ has an irreducible closed subspace $\mathcal{H} \neq\{0\}$. By definition of $\mathcal{V}$, there exists a $v \in V_{\kappa}^{(q)}$ such that the orthogonal projection of $\Psi_{H, \kappa}^{\Gamma}(v)$ to $\mathcal{H}$ is nonzero. Let $\tau_{\kappa}$ be the irreducible $K$-module with the highest weight $\kappa\left(\epsilon_{1}-\epsilon_{p}\right)$. Then $\tau_{\kappa}$ occurs not only in $\bigwedge^{q, q} \mathfrak{p}_{\mathbb{C}}^{*}$ by Proposition 11 but also in the $K$-module $\mathcal{H}$. Moreover, since $\Delta \Psi_{H, \kappa}^{\Gamma}=0$, the Casimir element $\Omega_{\mathfrak{g}}$ annihilates the space $\mathcal{H}$. Hence $\mathrm{H}^{q, q}\left(\mathfrak{g}_{\mathbb{C}}, K ; \mathcal{H}\right)=\operatorname{Hom}_{K}\left(\bigwedge^{q, q} \mathfrak{p}_{\mathbb{C}}, \mathcal{H}\right) \neq\{0\}$ ([1, Proposition 3.1(p.52)]). Then by [34, Theorem 4.1], there exists an element $H_{0} \in \sqrt{-1} \mathfrak{t}$ such that the $\left(\mathfrak{g}, K_{\mathbb{C}}\right)$-module $\mathcal{H}_{K}$ is isomorphic to $A_{\mathfrak{q}}$ with $\mathfrak{q}=\mathfrak{q}\left(H_{0}\right)$. Set $\mathfrak{u}=\mathfrak{u}\left(H_{0}\right)$ for simplicity. Since $T$-weight $\lambda(\mathfrak{q})$ is the highest weight of the unique $K$-type shared by $\wedge \mathfrak{p}_{\mathbb{C}}^{*}\left(\cong \bigwedge_{\mathbb{C}}\right)$ and $A_{\mathfrak{q}}([35$, Corollary 5.3, Theorem 5.6]), and since $\tau_{\kappa}$ occurs both in $\bigwedge^{q, q} \mathfrak{p}_{\mathbb{C}}^{*}$ and in $\mathcal{H}_{K} \cong A_{\mathfrak{q}}$, we must have $\lambda(\mathfrak{q})=\kappa\left(\epsilon_{1}-\epsilon_{p}\right)$.

Let $X$ be the set of eigenvalues of $\tau\left(H_{0}\right)$ acting on $\Lambda \mathfrak{p}_{\mathbb{C}}^{*}$. Since both $\lambda(\mathfrak{q})$ and $q\left(\epsilon_{1}-\epsilon_{p}\right)$ are $T$-weights of $\bigwedge \mathfrak{p}_{\mathbb{C}}^{*}$, the numbers $\left\langle\lambda(\mathfrak{q}), H_{0}\right\rangle$ and $\left\langle q\left(\epsilon_{1}-\epsilon_{p}\right), H_{0}\right\rangle$ belong to the set $X$.

If the space $\mathfrak{u} \cap \mathfrak{p}_{\mathbb{C}}$ were zero, then $\mathrm{H}^{0}\left(\mathfrak{g}_{\mathbb{C}}, K ; A_{\mathfrak{q}}\right) \neq 0$, which yields $A_{\mathfrak{q}} \cong \mathbb{C}$, a contradiction. This shows $\mathfrak{u} \cap \mathfrak{p}_{\mathbb{C}} \neq\{0\}$, which in turn implies $\left\langle\lambda(\mathfrak{q}), H_{0}\right\rangle>$ 0 , because the number $\left\langle\lambda(\mathfrak{q}), H_{0}\right\rangle$ is the maximal element of the set $X$ ([35, 
Proposition 5.2]). We have the inequality

$$
0<\left\langle\lambda(\mathfrak{q}), H_{0}\right\rangle=\kappa\left\langle\epsilon_{1}-\epsilon_{p}, H_{0}\right\rangle<q\left\langle\epsilon_{1}-\epsilon_{p}, H_{0}\right\rangle,
$$

which contradicts the maximality of the number $\left\langle\lambda(\mathfrak{q}), H_{0}\right\rangle$ in $X$ mentioned above.

The remaining is the primitive form $\Psi_{H, q}^{\Gamma}$, which can be regarded as the essential ingredient of the Poincaré dual form.

Lemma 39. The secondary spherical function $\psi_{s}=\varphi_{s}^{(q)}$ has a simple pole at $s=\rho_{0}$ with $\psi_{H}=\operatorname{Res}_{s=\rho_{0}} \psi_{s}$ such that

$$
\psi_{H}\left(a_{t}\right)=2 \frac{\Gamma\left(\rho_{0}+q\right)}{\Gamma\left(\rho_{0}+1\right) \Gamma(q-1)}(\cosh t)^{-2 \rho_{0}} \theta_{q}^{(q)}, \quad(\forall t>0) .
$$

The $(q, q)$-current $\psi_{H}$ is a harmonic form belonging to the space $\mathcal{A}^{q, q}(G / K)^{H}$. The coefficient functions $g \mapsto\left(\psi_{H}(g) \mid v\right)\left(v \in V_{q}^{(q)}\right)$ belong to $L^{2}(H \backslash G)$ and together with their right $U\left(\mathfrak{g}_{\mathbb{C}}\right)$ translates span a $\left(\mathfrak{g}_{\mathbb{C}}, K\right)$-submodule $\pi_{q}$ of $L^{2}(H \backslash G)$ isomorphic to $A_{\mathfrak{q}_{1 p}}$ with $\mathfrak{q}_{1 p}=\mathfrak{q}\left(q\left(\epsilon_{1}-\epsilon_{p}\right)\right)$.

Proof. The formula (8.1) follows directly from (5.1) by taking the residue. The formula (8.1) shows first that the singularity of $\psi_{s}$ along $H K$ vanishes in the level of the residue $\psi_{H}$, second that the $K$-type of $\psi_{H}$ is $q\left(\epsilon_{1}-\epsilon_{p}\right)$ (see Proposition 11). Moreover, from the equation in Proposition 23, $\psi_{H} \in \mathcal{A}^{q, q}(G / K)^{H}$ is harmonic, i.e., $\triangle \psi_{H}=0$. By a direct computation using the integration formula (2.3), we can easily confirm that $\psi_{H}$ is square-integrable on $H \backslash G$. From these properties, by the characterization of $A_{\mathfrak{q}_{1 p}}$ recalled above, we conclude that $\pi_{q}$ is $A_{\mathfrak{q}_{1 p}}$-isotypic. Irreducibility of $\pi_{q}$ follows from [33, Lemma 5.3] since the $K$-module $V_{q}^{(q)}$ is irreducible.

Proposition 40. Our global construction $\Psi_{H, q}^{\Gamma}$, if non-zero, yields an automorphic realization of $A_{\mathfrak{q}_{1 p}}$ in the space of $L^{2}$-automorphic forms $L^{2}(\Gamma \backslash G)$.

Proof. The $U\left(\mathfrak{g}_{\mathbb{C}}\right)$ translates of the coefficient functions of $\Psi_{H, q}^{\Gamma}$ spans a $\left(\mathfrak{g}_{\mathbb{C}}, K\right)$ submodule $\Pi$ of $L^{2}(\Gamma \backslash G)$; $\Pi$ contains the $K$-type $\tau_{q}$ and is annihilated by $\Omega_{\mathfrak{g}}$. Use the characterization of $A_{\mathfrak{q}_{1 p}}$ and [33, Lemma 5.3] to conclude $\Pi \cong A_{\mathfrak{q}_{1 p}}$.

Remark: It is a subtle and difficult arithmetic problem to find whether the primitive form $\Psi_{H, q}^{\Gamma}$ is zero or not for a fixed $\Gamma$. 
Non-vanishing statements of the Poincaré series constructed from an ordinary spherical function with regular spectral parameter (for small $\Gamma$ ) are found in Oshima [27] and Tong-Wang [33].

\section{REMARKS AND FURTHER OBSERVATIONS}

- Let us discuss the case when $G=\mathrm{U}(2,2), K=\mathrm{U}(2) \times \mathrm{U}(2)$ and $H=$ $\mathrm{U}(1,2) \times \mathrm{U}(1)$ in some detail. Since the complex dimension of the associated symmetric space $G / K$ is 4 , the fundamental class of the cycle $C_{H}^{\Gamma}$ is in the cohomology group $\mathrm{H}^{4}(\Gamma \backslash G / K ; \mathbb{C})$ of middle degree 4 . It is known that a non-trivial $\left(\mathfrak{g}_{\mathbb{C}}, K\right)$-modules contributing degree 4 cohomology group is a member of the discrete series representations with the same infinitesimal character as $\mathbb{C}$. By the classification, there exist 6 such representations $\pi(\Lambda)$, labeled by the Harish-Chandra parameters $\Lambda=\Lambda_{j}(j \in\{1,2,3,4,5,6\})$ :

$$
\begin{array}{lll}
\Lambda_{1}=\left(\frac{3}{2}, \frac{1}{2}, \frac{-1}{2}, \frac{-3}{2}\right), & \Lambda_{2}=\left(\frac{3}{2}, \frac{-1}{2}, \frac{1}{2}, \frac{3}{2}\right), & \Lambda_{3}=\left(\frac{3}{2}, \frac{-3}{2}, \frac{1}{2}, \frac{-1}{2}\right), \\
\Lambda_{4}=\left(\frac{1}{2}, \frac{-1}{2}, \frac{3}{2}, \frac{-3}{2}\right), & \Lambda_{5}=\left(\frac{1}{2}, \frac{-3}{2}, \frac{3}{2}, \frac{1}{2}\right), & \Lambda_{6}=\left(\frac{-1}{2}, \frac{-3}{2}, \frac{3}{2}, \frac{1}{2}\right) .
\end{array}
$$

The highest weight of the minimal $K$-type of the representation $\pi(\Lambda)$ is given by $\left[\lambda_{1}+1 / 2, \lambda_{2}-1 / 2\right] \oplus\left[\lambda_{3}-1 / 2, \lambda_{4}+1 / 2\right]$ if $\Lambda=\left(\lambda_{1}, \lambda_{2}, \lambda_{3}, \lambda_{4}\right)$. The representation $A_{\mathfrak{q}_{12}}$ defined in the last section is $\pi\left(\Lambda_{3}\right)$, which is one of two 'middle discrete series representations' $\pi\left(\Lambda_{3}\right)$ and $\pi\left(\Lambda_{4}\right)$ contributing the $(2,2)$ Hodge component of the cohomology group.

The $K$-type decomposition of $\Psi_{H}^{\Gamma}$ in this case is

$$
\Psi_{H}^{\Gamma}=\frac{\operatorname{vol}\left(\Gamma_{H} \backslash H / K_{H}\right)}{\operatorname{vol}(\Gamma \backslash G / K)} \frac{1}{12}\left(\omega_{\mathfrak{p}}+\gamma_{11}^{2}+\gamma_{22}^{2}+2 \gamma_{12} \wedge \gamma_{21}\right)+\Psi_{H, 2}^{\Gamma} .
$$

If the primitive form $\Psi_{H, 2}^{\Gamma}$ is nonzero, it generates a middle discrete series $\pi\left(\Lambda_{3}\right)$ in $L^{2}(\Gamma \backslash G)$. In order to investigate this form, the detailed knowledge about various spherical functions on $\mathrm{U}(2,2)$ ([7], [11]) should be basic.

- Though our global results after Proposition 33 in this paper are stated under the assumption that $\Gamma \backslash G$ is compact, the same statements (except a proper modification of the functional equation of $\left.\Phi_{s, r}^{(d)}\right)$ should be true for arithmetic non-uniform lattices $\Gamma$. But the situation is technically more sophisticated. 


\section{REFERENCES}

[1] Borel, A., Wallach, N., Continuous Cohomology, Discrete Subgroups, and Representations of Reductive Groups, The 2-nd ed. Mathematical Surveys and Monographs 67, Amer. Math. Soc., 2000.

[2] Bruinier, J., Borcherds products on $O(2, l)$ and Chern classes of Heegner divisors, Lecture Notes in Math. 1780, Springer, 2002.

[3] Coddington, E.A., Levinson, N., The theory of ordinary differential equations, McGraw-Hill Book Company, Inc., 1955.

[4] Funke, J., Milson, J., Cycles with local coefficients for orthogonal groups and vector-valued modular forms, Amer. J. Math. 128 (2006), 899-948.

[5] Gillet, H., Soulé, C., Arithmetic intersection theory, Publication of I.H.E.S. 72 (1990), 94174.

[6] Goodman, R., Wallach, N., Representations and Invariants of the Classical Groups, Cambridge University Press , 1998.

[7] Gon, Y., Generalized Whittaker functions on $S U(2,2)$ with respect to the Siegel parabolic subgroup, Mem. Amer. Math. Soc., 155 no. 738, 2002.

[8] Gon, Y., Tsuzuki, M., The resolvent trace formula for rank one Lie groups, Asian J. Math. 6 (2002), 227-252.

[9] Gradshteyn, I.S., Ryzhik, I.M., Tables of Integrals, Series and Products (the 6-th edition), Academic Press, Inc., 2000.

[10] Griffiths, P., Harris, J., Principles of Algebraic Geometry, Wiley Classics Library Edition, John Wiley \& sons, Inc. 1994.

[11] Hayata, T., Oda, T., Koseki, H., Matrix coefficients of the middle discrete series of $S U(2,2)$, J. Funct. Anal. 185 (2001), 297-341.

[12] Hirzebruch, F., Zagier, D., Intersection numbers of curves on Hilbert modular surfaces and modular forms of Nebentypus, Invent. Math. 36 (1976), 57-113.

[13] Kobayashi, T., and Oda, T., A vanishing theorem for modular symbols on locally symmetric spaces, Comm. Math. Helvetici 73, (1998), 45-70.

[14] Kudla, S.S, Milson, J.J, The theta correspondence and harmonic forms. I, Math. Ann. 274 (1986), 353-378.

[15] _ The theta correspondence and harmonic forms. II, Math. Ann. 277 (1987), 267314.

[16] Kudla, S.S, Central derivatives of Eisenstein series and height pairings, Ann. Math. 146 (1997), 545-646.

[17] Kudla, S.S.,Integrals of Borcherds forms, Compositio Math. 137 (2003), 293-349.

[18] Cohomology of arithmetic groups and automorphic forms (Luminy-Marseille, J.-P. Labesse and J. Schwermer, eds.), Lecture Notes in Math., 1447, Springer, Berlin, 1990.

[19] Magnus, W., Oberhettinger, F., Soni, R., Formulas and Theorems for the Special Functions of Mathematical Physics, in Einzeldarstellungen mit besonderer Berücksichtingung der Anwendungsgebiete Band 52, Springer-Verlag, NewYork, 1966. 
[20] Miattello, R., Wallach. N., Automorphic forms constructed from Whittaker vectors, J. Funct. Anal. 86, (1989), 411-487.

[21] (1992), 663-698.

[22] Oda, T., On modular forms associated with indefinite quadratic forms of signature $(2, n-2)$, Math. Ann. 231 (1977), 97-144.

[23] Oda, T., Periods of Hilbert modular surfaces, Progress in Math. Vol. 19, 123 p., Birkhäuser Boston, 1982.

[24] Oda, T., A note on a geometric version of the Siegel formula for quadratic forms of signature $(2,2 k)$, Science Reports of Niigata University, Series A, 20 (1984), 13-24.

[25] Oda, T., Hodge structures attached to geometric automorphic forms, Advanced Studies in Pure Math. 7 (1985), 223-276.

[26] Oda, T., Tsuzuki, M., Automorphic Green functions associated with the secondary spherical functions, Publ. RIMS, Kyoto Univ. 39 (2003), 451-533.

[27] Oshima, T., Asymptotic behavior of Flensted-Jensen's spherical trace functions with respect to spectral parameters, Algebraic analysis, geometry and number theory (Baltimore, MD 1988), 313-323, Johns Hopkins Univ. Press, 1989.

[28] Oshima, T., Sekiguchi, J., The restricted root system of a semisimple symmetric pair, Advanced Studies in Pure Math. 4 (1984), 433-497.

[29] Rossmann, W., The structure of semisimple symmetric spaces, Can. J. Math. 31 (1979), 157-180.

[30] Satake, I., Algebraic structures of symmetric domains, Publ. of the Math. Soc. of Japan 14, Iwanami Shoten, Publishers and Princeton Univ. Press, 1980

[31] Heckman, G., Schlichtkrull, H., Harmonic Analysis and Special Functions on Symmetric Spaces, Perspectives in Math. 16, Academic Press, Inc. (1994).

[32] Tsuzuki, M., Green currents for modular cycles in arithmetic quotients of complex hyperballs, Pacific J. Math., 227 (2006), 311-359.

[33] Tong, Y. L., Wang, S. P., Geometric realization of discrete series for semisimple symmetric spaces, Invent. Math. 96 (1989), 425-458.

[34] Vogan, D., Zuckerman, G., Unitary representations with nonzero cohomology, Compositio Math. 53 (1984), 51-90.

[35] Vogan, D., Cohomology and group representations, Proceeding of Symposia in Pure Mathematics, 61 (1997), 219-243.

[36] Wong, H., Dolbeault cohomological realization of Zuckerman module associated with finite rank representations, J. Func. Anal. 129 (1995), 428-454.

[37] Weil, A., Elliptic functions according to Eisenstein and Kronecker, Ergebnisse der Mathematik und ihrer Grenzgebiete 88, Springer-Verlag, 1976.

[38] _ Introduction a l'étude des variété kählériennes, Hermann, Paris, 1958.

[39] Wells, R.O., Differential Analysis on Complex Manifolds, GTM 65, Springer-Verlag, New York (1980) 
Takayuki Oda

Graduate School of Mathematical Sciences

The University of Tokyo, 3-8-1 Komaba Meguro-ku Tokyo, 153-8914, Japan

E-mail: takayuki@ms.u-tokyo.ac.jp

Masao Tsuzuki

Department of Mathematics

Sophia University, Kioi-cho 7-1 Chiyoda-ku Tokyo, 102-8554, Japan

E-mail: tsuzuki@mm.sophia.ac.jp 